\title{
Hypothalamic circuits for mechanically-evoked defensive attack
}

Peng Cao ( $\square$ caopeng@nibs.ac.cn )

National Institute of Biological Sciences, Beijing https://orcid.org/0000-0001-7739-6857

\section{Zhiyong Xie}

National Institute of Biological Sciences, Beijing

\section{Huating Gu}

National Institute of Biological Sciences, Beijing

\section{Congping Shang}

Bioland Laboratory, Guangdong

\section{Xinyu Cheng}

National Institute of Biological Sciences, Beijing

\section{Meizhu Huang}

National Institute of Biological Sciences, Beijing

\section{Dapeng Li}

National Institute of Biological Sciences, Beijing

\section{Ting Tao}

National Institute of Biological Sciences, Beijing

\section{Yuan Xie}

Hebei Medical University

Jidong Zhao

Institute of Zoology, CAS

Wei Lu

Institute of Zoology, CAS

\section{Zhibing Zhang}

Institute of Zoology

\section{Zongxiang Tang}

Nanjing University of Chinese Medicine

Cheng Zhan

National Institute of Biological Sciences, Beijing https://orcid.org/0000-0001-9397-0871

\section{Fan Zhang}

Hebei Medical University 
Keywords: Innate Defensive Behaviors, Threat-relevant Sensory Information, Noxious Mechanical Force, GABAergic Brain Circuit

Posted Date: November 14th, 2020

DOI: https://doi.org/10.21203/rs.3.rs-102096/v1

License: (c) (i) This work is licensed under a Creative Commons Attribution 4.0 International License. Read Full License

Version of Record: A version of this preprint was published at Nature Neuroscience on January 3rd, 2022. See the published version at https://doi.org/10.1038/s41593-021-00985-4. 
Title: Hypothalamic circuits for mechanically-evoked defensive attack

Authors: Zhiyong $\mathrm{Xie}^{1, \#}$, Huating $\mathrm{Gu}^{1,2, \#}$, Congping Shang ${ }^{3}$, Xinyu Cheng ${ }^{1,4}$, Meizhu Huang $^{3}$, Dapeng $\mathrm{Li}^{1}$, Ting Tao ${ }^{1}$, Yuan $\mathrm{Xie}^{5}$, Jidong Zhao ${ }^{6}$, Wei $\mathrm{Lu}^{6}$, Zhibing Zhang ${ }^{6}$, Zongxiang Tang ${ }^{7}$, Cheng Zhan ${ }^{1,8}$, Fan Zhang ${ }^{5,{ }^{*}}$, Peng Cao ${ }^{1,8,{ }^{*}}$

\section{Affiliation:}

${ }^{1}$ National Institute of Biological Sciences, Beijing 102206, China

${ }^{2}$ College of Life Sciences, Beijing Normal University, Beijing 100875, China

${ }^{3}$ Bioland Laboratories, Guangzhou, Guangdong 510005, China

${ }^{4}$ Graduate School of Peking Union Medical College, Chinese Academy of Medical Sciences, Beijing, China

${ }^{5}$ Key Laboratory of Neural and Vascular Biology in Ministry of Education, Department of Pharmacology, Hebei Medical University, Shijiazhuang, Hebei, China.

${ }^{6}$ State Key Laboratory of Integrated Management of Pest Insects and Rodents, Institute of Zoology, Chinese Academy of Sciences, Beijing 100101, China

7 School of Medicine and Holistic Integrative Medicine, Nanjing University of Chinese Medicine, Nanjing, Jiangsu 210023, China

${ }^{8}$ Tsinghua Institute of Multidisciplinary Biomedical Research, Tsinghua University, Beijing 100084, China

\# Co-first authors

*Correspondence: caopeng@nibs.ac.cn (P.C.) or zhangfan86@hebmu.edu.cn (F.Z.) 


\section{ABSTRACT}

The innate defensive behaviors triggered by environmental threats play a critical role in animal survival. Among these behaviors, defensive attack physically toward threatening target (e.g. predator) is the last line of defense to struggle for survival. How the brain transforms threat-relevant sensory information into the action of defensive attack remains poorly understood. We found that noxious mechanical force in an inescapable context was a key stimulus to trigger defensive attack in laboratory mice. The mechanically-evoked defensive attack was abrogated by photoinhibition of vGAT+ neurons in the anterior hypothalamic nucleus (AHN). The AHN vGAT+ neurons encoded the intensity of mechanical force and were innervated by brain areas related to pain and attack. Activation of these neurons triggered biting attack toward predator, while suppressing other ongoing behaviors. The efferent pathway from AHN vGAT+ neurons to the periaqueductal gray was both sufficient and necessary for mechanically-evoked defensive attack. Together, these data revealed a GABAergic brain circuit engaged in converting noxious mechanical stimuli to neural signals that provoke defensive attack in mice. (168 words) 
In response to environmental threats, human and animals exhibit a cascade of innate defensive behaviors (e.g. freezing, escape, and defensive attack). These behaviors may occur as a function of physical distance of threats, as described by a classical model termed "Predatory Imminence Continuum" by Fanselow and colleagues (Fanselow and Lester, 1988). This model has been well supported by recent behavioral studies. For example, when prey detects a distant cruising predator, freezing is usually the dominant form of post-encounter defensive behavior of prey (De Franceschi et al., 2016). In response to looming visual stimuli mimicking an approaching predator, prey rapidly escapes to avoid prey capture (Yilmaz and Meister, 2013). If a cornered prey is physically attacked by predator, defensive attack of prey is often provoked as the last line of defense to struggle for survival (Blanchard and Blanchard, 1989). Relative to the well characterized brain circuits for freezing and escape (Hypothalamus: Silva et al., 2013; Wang et al., 2015; Kunwar et al., 2015; Midbrain: Wei et al., 2015; Shang et al., 2015; Tovote et al. 2016; Evans et al., 2018; Shang et al., 2018; Thalamus: Salay et al., 2018; Pons: Han et al., 2015; Review: Gross and Canteras, 2012; Branco and Redgrave, 2020), the brain mechanisms for sensory-triggered defensive attack is poorly understood. In the present study, we explored how the brain transforms threat-relevant sensory information into the action of defensive attack in mice.

\section{Noxious mechanical stimulus to provoke defensive attack in mice}

We began this study by measuring defensive attack behavior in a rodent-versus-snake paradigm (Paschoalin-Maurin et al., 2018; Mendes-Gomes et al., 2020). In an enclosed arena $(10 \mathrm{~cm} \times 10 \mathrm{~cm})$, a male C57BL/6 mouse was exposed to a weight-matched young snake (Elaphe schrenckii) (Figure S1A). When the snake attacked the mouse, the mouse exhibited jumping escape and then biting-like defensive attack to the snake (Movie S1). We also observed biting-like defensive attack in rat (Figure S1B, Movie S2) and hamster (Figure S1C, Movie S3) when they were physically 
attacked by the snake. These observations indicated that rodents exhibit robust anti-predator defensive attack behavior in laboratory conditions.

To identify the key stimulus to provoke defensive attack, we performed three experiments using mouse as an animal model. First, we examined the defensive responses of mice to a plastic dummy snake, which was either coated with snake feces to provide predator-derived olfactory cues or equipped with an alligator-clip to apply noxious mechanical stimuli (Figure 1A). Predator-derived olfactory cues on the dummy snake did not provoke defensive attack (Movie S4; Figure 1B, 1C \& 1E) but promoted freezing, risk assessment and avoidance in mice (Figure S2, A-C). When the alligator-clip on the dummy snake applied sustained mechanical force (366 grams) by clamping the tail of mice, they exhibited biting attacks toward the dummy snake (Movie S5; Figure 1D \& 1F) without showing freezing, risk assessment or avoidance (Figure S2, D-F). The defensive attack provoked by the alligator-clip clamping on the tail was similarly observed in the light ( $\sim 50$ lux) and dark ( $\sim 0.002$ lux) conditions, suggesting visual detection of the alligator clip clamping the tail may play a minor role in provoking such defensive attack (Figure S2, G-I). The noxious mechanical stimuli applied on the limbs of mice also induced robust biting attack toward dummy snake (Figure S2J). Second, we found that the mice also exhibited biting-like attacks to neutral objects when these objects were physically connected to noxious mechanical stimulus (Movie S6; plastic lid: Figure S3A; wood block: Figure S3B). These data again underscored the role of noxious mechanical force as a general stimulus to trigger defensive attack. Third, we performed genetic ablation of Mrgprd+ sensory neurons, which may mediate behavioral responses to noxious mechanical stimuli (Cavanaugh et al., 2009), by expressing diphtheria toxin receptor (DTR) in Mrgprd+ neurons. In the Mrgprd-CreERT2/iDTR/Ai3 mice (Olson et al., 2017; Buch et al., 2005; Madisen et al., 2010), tamoxifen injections and subsequent diphtheria toxin injections decreased $(86 \% \pm 7 \%, n=7$ pairs) the number of EYFP+ neurons (putatively Mrgprd+) in the dorsal root ganglion (Figure 1G). 
This genetic manipulation significantly reduced biting attack in mice with the tail or limb clamped by the alligator-clip (Figure $1 \mathrm{H}$ and $1 \mathrm{I}$ ). Together, these three lines of evidence suggested that noxious mechanical force may be a key stimulus to evoke defensive attack in mice.

\section{AHN vGAT+ neurons are required for mechanically-evoked defensive attack}

Then we explored the brain circuits for mechanically-evoked defensive attack. Earlier studies reported that the medial hypothalamic zone ( $\mathrm{MHZ}$ ) plays a critical role in the expression of defensive behaviors, especially with respect to predators (Canteras et al., 1997; Canteras et al., 2002). The MHZ includes the anterior hypothalamic nucleus $(\mathrm{AHN})$, dorsomedial part of ventromedial hypothalamic nucleus (VMHdm) and dorsal premammillary nucleus (PMd). Neurons in the VMHdm and PMd participate in defensive behaviors such as freezing and escape (Silva et al., 2013; Wang et al., 2015; Kunwar et al., 2015; Cezario et al., 2008). The AHN may participate in intraspecific aggression (Rat: Olivier et al., 1983; Cat: Fuchs et al., 1985; Hamster: Delville et al., 2000; Prairie Voles: Gobrogge et al., 2007; Finch: Goodson et al., 2012). However, the role of AHN in anti-predator defensive behavior remains unknown. A recent study demonstrated that the rats threatened by snakes showed robust increase in c-Fos expression in the AHN (Paschoalin-Maurin et al., 2018), prompting us to test the hypothesis that AHN neurons may be involved in mechanically-evoked defensive attack in mice.

Fluorescent in situ hybridization analyses (Figure S4A-S4C) indicated that the AHN neurons are predominantly vGAT+ $(92 \% \pm 3.6 \%, \mathrm{n}=3$ mice $)$, with only a small proportion are vGlut $2+(8 \% \pm 0.7 \%, n=3$ mice $)$. To explore the contributions of vGAT+ and vGlut2+ AHN neurons to mechanically-evoked defensive attack, we employed vGAT-IRES-Cre and vGlut2-IRES-Cre mice to genetically manipulate these AHN neurons (Vong et al., 2011). The specificity and efficiency of these Cre lines to label vGAT+ and vGlut2+ AHN neurons were confirmed in control experiments (Figure S4D-S4O). We injected AAV-DIO-GtACR1-2A-EGFP (Govorunova et al., 2015) into the AHN of vGAT-IRES-Cre 
and vGlut2-IRES-Cre mice, followed by optical fiber implantation above the AHN bilaterally (Figure 2A; Figure S5A and S5B). The cell-type specificity of GtACR1-2A-EGFP expression was confirmed in control experiments (Figure S5C-S5F). The effect of photoinhibition on AHN neurons expressing GtACR1 was validated in acute brain slices (Figure 2B). By recording electromyogram (EMG) from masseter muscles in freely moving mice (Falkner et al., 2020) (Figure 2C), we were able to measure the initiation and termination of biting bouts during defensive attack (Figure S5G). We found that photoinhibition of AHN vGAT+ neurons $(473 \mathrm{~nm}, 2 \mathrm{~s}-\mathrm{OFF} / 2 \mathrm{~s}-\mathrm{ON}, 10 \mathrm{~mW}$ ) rapidly (latency $=0.82 \mathrm{~s} \pm 0.12 \mathrm{~s} ; \mathrm{n}=7$ mice) terminated biting attack provoked by noxious mechanical stimuli (Movie S7; Figure 2D-2F). In contrast, photoinhibition of AHN vGlut2+ neurons did not prevent mechanically-evoked defensive attack (Figure 2G-2I). As a control experiment, photoinhibition of AHN vGAT+ neurons did not alter average locomotion speed of freely-moving mice (Figure $\mathrm{S} 5 \mathrm{H}$ ). These data suggested that the AHN vGAT+ neurons are selectively required for mechanically-evoked defensive attack in mice.

\section{AHN vGAT+ neurons encode mechanical stimuli}

To test whether AHN vGAT+ neurons respond to noxious mechanical stimuli, we expressed jGCaMP7s in these neurons and recorded GCaMP fluorescence with fiber photometry (Gunaydin et al., 2014; Dana et al., 2019) (Figure 3A; Figure S6A and S6B). In head-fixed mice standing on a treadmill, noxious mechanical stimulus applied by the alligator-clip to mouse tail robustly increased the GCaMP fluorescence (Figure 3B). However, in other behavioral tests (biting attack, locomotion, risk assessment, object exploration and social investigations), the GCaMP fluorescence was only modestly increased (Figure S6C-S6H; Figure 3C). These data suggested that AHN vGAT+ neurons may preferentially respond to noxious mechanical stimuli.

To test whether single AHN vGAT+ neurons encode mechanical stimuli, we expressed ChR2-mCherry in these neurons and performed single-unit recording with an 
optrode in the AHN of head-fixed awake mice (Figure 3D; Figure S7A). The putative vGAT+ AHN neurons were identified according to the action potentials (APs) evoked by light pulses (473 nm, $1 \mathrm{~ms}, 10 \mathrm{~mW}$ ) illuminating on ChR2-mCherry ${ }^{+} \mathrm{AHN}$ neurons. The light-evoked APs had to conform to two criteria: first, their latencies to the light pulses should be less than $5 \mathrm{~ms}$; second, their waveforms should be similar to those of sensory-evoked APs (Cohen et al., 2012). With these empirical criteria, we identified 15 units as putative AHN vGAT+ neurons. Their light-evoked APs had short response latencies $(2.7 \mathrm{~ms} \pm 0.4 \mathrm{~ms}, \mathrm{n}=15$ units; Figure $3 \mathrm{E})$ and possessed waveforms quantitatively correlated with those of mechanically-evoked APs (Figure S7B; Figure 3F).

Then we examined the responses of these 15 putative AHN VGAT+ neurons to mechanical and olfactory stimuli. Mechanical stimuli were applied by an alligator-clip clamping on mouse tail (Figure S7C and S7D). Snake feces coated on a cotton swab was used to provide olfactory stimuli of predator (Figure S7E and S7F). The 15 putative AHN vGAT+ neurons responded to both mechanical stimuli and olfactory stimuli (Figure $3 G)$. However, the responses to mechanical stimuli were significantly stronger than those to olfactory stimuli of predator (Figure $3 \mathrm{H}$; One-Way ANOVA, $\mathrm{P}<0.001$ ). These data confirmed the GCaMP results that vGAT+ AHN neurons preferentially respond to mechanical stimuli.

To quantitatively test how AHN vGAT+ neurons encode mechanical force, we used von Frey filaments $(1 \mathrm{~g}, 10 \mathrm{~g}$, and $100 \mathrm{~g}$ ) to poke different body parts of mice (Figure S7G). The 15 putative AHN vGAT+ neurons responded to von Frey filaments poking on the tail in a graded manner (Figure $3 \mathrm{l}$ and $\mathrm{S} 7 \mathrm{H}$ ) and preferred stronger mechanical force (1g vs. 10g: $P<0.001 ; 10 \mathrm{~g}$ vs. 100g: $\mathrm{P}<0.001 ; \mathrm{n}=15$ units, One-Way ANOVA; Figure 3J). Moreover, these neurons also responded to mechanical stimuli delivered to four limbs, with a bias toward the contralateral side (Figure 3K). They exhibited clear adaptation in response to repetitive mechanical stimuli at $0.5 \mathrm{~Hz}$ (Figure $3 \mathrm{~L}$ ). Recording sites marked 
by electrolytic lesion were all located within the AHN (Figure S7I). Together, these data suggested that AHN vGAT+ neurons encode mechanical stimuli on the body surface.

\section{Mapping monosynaptic inputs of AHN vGAT+ neurons}

With recombinant rabies virus (RV), we next performed monosynaptic retrograde tracing (Wickersham et al., 2007) to examine how AHN vGAT+ neurons are connected with brain areas associated with mechanical stimuli or attack behavior (Figure S8A-S8C). A brain-wide survey revealed a number of monosynaptic projections to the AHN vGAT+ neurons (Figure S8D and S8E). First, robust monosynaptic inputs arise from the lateral parabrachial nucleus (LPB) and paraventricular thalamic nucleus (PVT) (Figure S8, D1 and D2), two brain areas that were directly innervated by TAC1+ spinal neurons for pain-related defensive behaviors (Huang et al., 2019). Second, AHN vGAT+ neurons are monosynaptically innervated by neurons in the $\mathrm{VMH}$ and lateral septal nucleus (LS) (Figure S8, D3 and D4), both of which have been related to attack behaviors in mice (VMH: Lin et al., 2011; LS: Leroy et al., 2018). Third, consistent with the observation that AHN vGAT+ neurons modestly responded to predator-derived olfactory cues, we found that these neurons receive sparse innervations from the medial amygdala (MA) (Figure S8, D3), which convey olfactory signals from the accessory olfactory bulb to hypothalamic nuclei (Isogai et al., 2011). These data supported the hypothesis that AHN vGAT+ neurons are within a brain network for mechanically-evoked defensive attack.

\section{AHN vGAT+ neurons trigger biting attacks toward non-social targets}

Then we tested whether activation of AHN vGAT+ neurons is sufficient to trigger defensive attack behavior. We injected AAV-DIO-ChR2-mCherry into the AHN of vGAT-IRES-Cre mice, followed by implantation of optical fibers above the AHN (Figure 4A). The cell-type specificity of ChR2-mCherry expression in AHN vGAT+ neurons was confirmed in control experiments (Figure 4B and S9A). The effectiveness of photostimulation to trigger action potential firing in ChR2-mCherry+ AHN neurons was 
validated in acute brain slices (Figure 4C). When the test mice were confronted with a live snake in the arena (Figure 4D), the mice exhibited a series of defensive responses (risk assessment, avoidance, and freezing); light-stimulation of AHN vGAT+ neurons rapidly switched mouse behavior to biting-like attack toward the snake (Movie S8; Figure 4E). Quantitative measurement of time spent on these behaviors indicated that light-stimulation rapidly evoked biting-like attack toward the snake (latency $=2.7 \mathrm{~s} \pm 0.4 \mathrm{~s}$, $\mathrm{n}=7$ mice; Figure 4F) and suppressed other defensive behaviors (Risk assessment: Figure 4G; Avoidance: Figure S9B; Freezing: Figure S9C). Light-evoked biting attack was a function of the frequency and laser power of light stimulation (Figure S9D and S9E). The biting actions toward snake in the attack episodes were confirmed by EMG recording (Movie S9; Figure S9F and S9G). The same photostimulation also evoked biting-like attack to the wood block (Movie S10; Figure S9H-S9K), which was reminiscent of the earlier observation that neutral targets linked to noxious mechanical stimuli also evoked defensive attack (Figure S3).

One important concern about the specificity of biting-like attack evoked by AHN vGAT+ neurons may be raised. Activation of AHN vGAT+ neurons may broadly provoke an aggressive state that drives the mice to attack any targets without target-specificity. To address this concern, we examined how light-stimulation of AHN vGAT+ neurons affects social aggression. Unexpectedly, when the test mouse in its homecage exhibited social aggression to a male intruder (Figure $4 \mathrm{H}$ ), light-stimulation of AHN vGAT+ neurons strongly suppressed ongoing social attack (Movie S11; Figure 4I and 4J). In addition, in male mice without showing social aggression, light-stimulation of AHN vGAT+ neurons did not evoke social attack to male or female conspecifics (Male: Movie S12; Figure S10A-S10D; Female: Movie S13; Figure S10F-S10I). These results suggested that activation of AHN vGAT+ neurons induced biting-like attack selectively to non-social targets. 
Although light-stimulation of AHN vGAT+ neurons did not evoke social attack, it increased the time for social investigation to conspecifics (Figure 4K, S10E and S10J). To measure the priority level of light-evoked biting attack to predator and social investigation to conspecifics, we performed two additional experiments. First, in an arena with both snake and male conspecifics (Figure 4L), the test mice actively investigated the male conspecifics before light-stimulation; light-stimulation of AHN vGAT+ neurons rapidly switched mouse behavior from social investigation to biting-like attack toward the snake (Movie S14; Figure 4M-40; Figure S11A-S11D). Second, in the presence of both snake and female conspecifics in the arena (Figure 4P), the test mice spent considerable time in typical courtship behaviors toward the female before light-stimulation; light-stimulation of AHN vGAT+ neurons rapidly switched mouse behavior to biting-like attack toward predator (Movie S15; Figure 4Q-4S; Figure S11E-S11H). Thus, the anti-predator defensive attack evoked by light-stimulation of AHN vGAT+ neurons has a higher priority than social investigation.

\section{The vGAT+ AHN-PAG pathway for mechanically-evoked defensive attack}

Then we explored the efferent pathways of AHN vGAT+ neurons involved in defensive attack, by injecting AAV-DIO-EGFP-Syb2 into the AHN of vGAT-IRES-Cre mice (Figure S12A and S12B). Consistent with an earlier study (Risold et al., 1994), we found that the AHN vGAT+ neurons divergently projected to different brain regions ipsilaterally, including MPOA, LS, VMH, PMd, VIPAG, and other areas (Figure S12C-S12G). Both the VIPAG and LS have been implicated in attack-related behaviors in mice (vIPAG: Park et al., 2018; Falkner et al., 2020; LS: Leroy et al., 2018). Thus, we tested whether activation of vGAT+ AHN-vIPAG or AHN-LS pathway would evoke defensive attack behavior. AAV-DIO-ChR2-mCherry was injected into the AHN of vGAT-IRES-Cre mice, followed by optical fibers implanted above ChR2-mCherry+ axon terminals in the vIPAG (Figure 5A; Figure S13A and S13B). Light stimulation of vGAT+ AHN-vIPAG pathway reliably provoked biting-like attack toward live snake (Figure 5B 
and 5C) and suppressed other defensive behaviors (Figure S13C-S13E). In contrast, activation of VGAT+ AHN-LS pathway failed to induce biting-like attack toward snake (Figure S14A-S14H). These data suggested that vGAT+ AHN-vIPAG pathway may be the primary pathway to provoke anti-predator defensive attack in mice.

To rule out the possibility of collateral activation, we examined whether the anti-predator attack behavior triggered by AHN vGAT+ neurons could be blocked by the antagonist of GABAa receptor (picrotoxin, PTX) infused in the VIPAG. AAV-DIO-ChR2-mCherry was injected into the AHN of vGAT-IRES-Cre mice, followed by implanting an optical fiber above the AHN and cannulae above the vIPAG (Figure 5D; Figure S15A and S15B). In acute brain slices, perfusion of PTX effectively abrogated light-evoked GABAergic postsynaptic currents in vIPAG neurons (Figure S15C; Figure $5 E$ and $5 F)$. In behaving mice, delivery of PTX (100 $\mu \mathrm{M})$ through cannulae (Figure S15D) blocked the light-evoked anti-predator attack in a dose-dependent manner (Figure S15E and S15F; Figure 5G). As a control, delivery of saline did not alter light-evoked anti-predator attack (Figure $5 \mathrm{H}$ ).

To determine whether vGAT+ AHN-vIPAG pathway is required for mechanically-evoked defensive attack, we injected AAV-DIO-GtACR1-2A-EGFP in the AHN of VGAT-IRES-Cre mice, followed by optical fiber implantation above the vIPAG (Figure 5l; Figure S16A and S16B). Photoinhibition of VGAT+ AHN-vIPAG pathway significantly impaired mechanically-evoked defensive attack behavior (Figure S16C; Figure $5 \mathrm{~J}$ and $5 \mathrm{~K}$ ). In control experiment, light illumination on EGFP-expressing axon terminals of AHN vGAT+ neurons in the vIPAG did not change mechanically-evoked defensive attack (Figure S16C; Figure $5 \mathrm{~J}$ and $5 \mathrm{~K}$ ). These results suggested that the vGAT+ AHN-vIPAG pathway is required for mechanically-evoked defensive attack in mice. 


\section{DISCUSSION}

In response to environmental threats, human and animals exhibit a cascade of innate defensive behaviors (e.g. freezing, escape, and defensive attack). Relative to the well characterized neural circuits for freezing and escape, the brain mechanisms underlying defensive attack are poorly understood. In the present study, we explored how the brain transforms threat-relevant sensory information into the action of defensive attack in mice.

\section{Noxious mechanical stimuli to provoke defensive attack}

By using a dummy snake combined with different sensory stimuli, we demonstrated that noxious mechanical force is a key stimulus to evoke defensive attack (Figure 1). The pivotal role of noxious mechanical force in defensive attack was further supported by the reduction of biting attacks in mice with genetic ablation of Mrgprd+ sensory neurons, which may mediate behavioral responses to noxious mechanical stimuli (Cavanaugh et al., 2009). By contrast, snake feces coated on the dummy snake to provide predator-derived olfactory cues (Isogai et al., 2011) failed to evoke defensive attack. Instead, the olfactory cues promoted other defensive responses (e.g. freezing, risk assessment and avoidance), an observation consistent with previous studies (Papes et al., 2010; Wang et a., 2018).

\section{AHN vGAT+ neurons are critical for mechanically-evoked defensive attack}

The MHZ has been proposed to play a critical role in anti-predator defensive behaviors (Canteras et al., 1997; Canteras et al., 2002), thus prompting us to seek for mechanisms underlying mechanically-evoked defensive attack in this brain area. We found that the activities of AHN VGAT+ neurons in the MHZ are selectively required for mechanically-evoked defensive attack (Figure 2). These neurons optimally responded to noxious mechanical stimuli, and their activities encode the intensity of mechanical force delivered onto the contralateral side of the body (Figure 3). Then why do these neurons respond to noxious mechanical stimuli? We found that $A H N$ VGAT+ neurons were 
monosynaptically innervated by the LPB and PVT (Figure S8), two brain areas directly innervated by TAC1+ spinal neurons for pain-related defensive behaviors (Huang et al., 2019). Together, these results suggested that AHN vGAT+ neurons may be a critical circuit module for mechanically-evoked defensive attack.

\section{Activation of AHN vGAT+ neurons evokes biting attack to non-social targets}

To further test the role of AHN vGAT+ neurons in defensive attack, we systematically measured photostimulation-induced mouse behaviors to a series of experimental targets. Light-stimulation of AHN vGAT+ neurons evoked biting attack toward predator (Figure 4F) and neutral object (Figure S9J). However, activation of these neurons failed to evoke social attack toward conspecifics (Figure S10); on the contrary, their activation suppressed social attack to male intruder in the paradigm of social aggression (Figure $4 \mathrm{~J})$. These observations indicated that activation of AHN vGAT+ neurons has not broadly provoked an aggressive state that drives the mice to attack any targets without target-specificity. In addition, these observations may be explained by our anatomical finding that one efferent projection of AHN vGAT+ neurons terminates in the VMHvl (inset of Figure S12E). The AHN vGAT+ neurons may presumably exert GABAergic inhibition to VMHvl neurons, which are causally linked to social aggression (Lin et al., 2011; Lee et al., 2014; Falkner et al., 2016; Yang et al., 2017).

Another interesting observation is that activation of AHN vGAT+ neurons triggered biting attack toward predator, while suppressing other forms of defensive behavior, such as risk assessment (Figure $4 \mathrm{G}$ ), avoidance (Figure S9B) and freezing (Figure S9C). These results suggested that AHN VGAT+ neurons may prioritize defensive attack above other forms of defensive behavior by a "brake" mechanism. Indeed, we found that AHN VGAT+ neurons project to the VMHdm and PMd (Figure S12, E and F). It is likely that AHN VGAT+ neurons may exert GABAergic inhibition to neurons in the VMHdm and PMd, which are involved in defensive behaviors such as freezing and avoidance (Silva et al., 2013; Wang et al., 2015; Kunwar et al., 2015; Cezario et al., 2008). 


\section{New Questions}

Our results also raise several new questions. First of all, although noxious mechanical stimuli optimally activated vGAT+ AHN neurons, predator-derived olfactory cues also modulated their activities (Figure $3 \mathrm{G}$ and $3 \mathrm{H}$ ). Moreover, these neurons receive sparse monosynaptic innervations from the MA (Figure S8D3), which convey olfactory signals from the accessory olfactory bulb to the hypothalamus (Isogai et al., 2011). How AHN vGAT+ neurons participate in olfaction-mediated defensive behaviors would be an interesting topic to pursue in future study.

Second, our results do not rule out the role of AHN vGAT+ neurons in social behavior. Earlier studies using lesion and electrical stimulation techniques suggested that the AHN in other species may participate in intraspecific aggression (Rat: Olivier et al., 1983; Cat: Fuchs et al., 1985; Hamster: Delville et al., 2000; Prairie Voles: Gobrogge et al., 2007; Finch: Goodson et al., 2012). In this study, optogenetic activation of vGAT+ AHN neurons significantly increased time for social investigation in a social context (Figure 4K; Figure S10, E and J). The exact role of AHN vGAT+ neurons in social behaviors of mice needs to be tested in future study.

Finally, vGAT+ neurons represent a large neuronal population in the AHN. In future study, it would be important to identify more specific genetic markers to define subtypes of AHN vGAT+ neurons by using single-cell RNA sequencing approach. With Cre lines associated with more specific genetic markers, one would be able to better dissect how AHN vGAT+ neurons mediate mechanically-evoked defensive attack. 


\section{SUPPLEMENTARY INFORMATION}

Supplementary information includes Methods, 16 figures, 4 tables and 15 movies.

\section{AUTHOR CONTRIBUTIONS}

P.C., C.Z., Z.Z., F.Z. conceived the study. Z.X. did injections and fiber implantation. H.G., X.C., T.T., Y.X. did behavioral tests. C.S., H.G. did EMG recording. H.G. did fiber photometry recording. Z.X., X.C., M.H. did histological analyses. M.H. maintained the snake. C.S. and H.G. did slice physiology. J.Z., W.L., Z.Z., F.Z., Z.T. provided reagents. D.L., C.S., H.G., Z.X., and P.C. analyzed data. P.C. wrote the manuscript.

\section{ACKNOWLEDGMENTS}

We thank Drs. Thomas Südhof, Karl Deisseroth and Miao He for providing plasmids and mouse lines. We also thank the members of the Neuroscience Pioneer Club for valuable discussions. This work was supported by the National Natural Science Foundation of China (31925019 to P.C. and 31872788 to F.Z.), the Natural Science Foundation of Hebei Province (C2020206027 to F.Z.), and the institutional grants from the Chinese Ministry of Science and Technology to NIBS. All data are archived in NIBS.

\section{COMPETING FINANCIAL INTERESTS}

The authors declare no competing financial interests. 


\section{REFERENCES}

1. Blanchard RJ, Blanchard DC. Attack and defense in rodents as ethoexperimental models for the study of emotion. Prog Neuropsychopharmacol Biol Psychiatry.13 Suppl:S3-S14. (1989)

2. Branco, T. \& Redgrave, P. The Neural Basis of Escape Behavior in Vertebrates. Annu Rev Neurosci 43, 417-439. (2020).

3. Buch, T. et al. A Cre-inducible diphtheria toxin receptor mediates cell lineage ablation after toxin administration. Nature Methods 2, 419-426. (2005).

4. Canteras, N. S. The medial hypothalamic defensive system: hodological organization and functional implications. Pharmacol Biochem Behav 71, 481-491. (2002).

5. Canteras, N. S., Chiavegatto, S., Ribeiro do Valle, L. E. \& Swanson, L. W. Severe reduction of rat defensive behavior to a predator by discrete hypothalamic chemical lesions. Brain Res Bull 44, 297-305 (1997).

6. Cavanaugh, D. J. et al. Distinct subsets of unmyelinated primary sensory fibers mediate behavioral responses to noxious thermal and mechanical stimuli. Proc Natl Acad Sci U S A 106, 9075-9080 (2009).

7. Cezario, A. F., Ribeiro-Barbosa, E. R., Baldo, M. V. \& Canteras, N. S. Hypothalamic sites responding to predator threats--the role of the dorsal premammillary nucleus in unconditioned and conditioned antipredatory defensive behavior. Eur J Neurosci 28, 1003-1015 (2008).

8. Chen, J. et al. A Vagal-NTS Neural Pathway that Stimulates Feeding. Current Biology (2020).

9. Cohen, J. Y., Haesler, S., Vong, L., Lowell, B. B. \& Uchida, N. Neuron-type-specific signals for reward and punishment in the ventral tegmental area. Nature 482, 85-88 (2012).

10. Dana, $\mathrm{H}$. et al. High-performance calcium sensors for imaging activity in neuronal populations and microcompartments. Nature methods 16, 649-657 (2019).

11. De Franceschi, G., Vivattanasarn, T., Saleem, A. B. \& Solomon, S. G. Vision Guides Selection of Freeze or Flight Defense Strategies in Mice. Curr Biol 26, 2150-2154 (2016).

12. Delville, Y., De Vries, G. J. \& Ferris, C. F. Neural connections of the anterior hypothalamus and agonistic behavior in golden hamsters. Brain Behav Evol 55, 53-76 (2000).

13. Evans, D. A. et al. A synaptic threshold mechanism for computing escape decisions. Nature 558, 590-594 (2018).

14.Falkner, A. L., Grosenick, L., Davidson, T. J., Deisseroth, K. \& Lin, D. Hypothalamic control of male aggression-seeking behavior. Nature neuroscience 19, 596-604 (2016).

15. Falkner, A. L. et al. Hierarchical Representations of Aggression in a Hypothalamic-Midbrain Circuit. Neuron 106, 637-648 (2020).

16. Fanselow, M. S. and Lester, L.S., (1988), A functional behavioristic approach to 
aversively motivated behavior: Predatory imminence as a determinant of the topography of defensive behavior, In R, C, Bolles \& M. D, Beecher (Eds.), Evolution and learning (pp, 185211). Hillsdale, NJ: Erlbaum,

17. Fuchs, S. A., Edinger, H. M. \& Siegel, A. The role of the anterior hypothalamus in affective defense behavior elicited from the ventromedial hypothalamus of the cat. Brain Res 330, 93-107 (1985).

18. Gobrogge, K. L., Liu, Y., Jia, X. \& Wang, Z. Anterior hypothalamic neural activation and neurochemical associations with aggression in pair-bonded male prairie voles. The Journal of Comparative Neurology 502, 1109-1122 (2007).

19.Goodson, J. L., Kelly, A. M., Kingsbury, M. A. \& Thompson, R. R. An aggression-specific cell type in the anterior hypothalamus of finches. Proc Natl Acad Sci U S A 109, 13847-13852 (2012).

20. Govorunova, E. G., Sineshchekov, O. A., Janz, R., Liu, X. \& Spudich, J. L. NEUROSCIENCE. Natural light-gated anion channels: A family of microbial rhodopsins for advanced optogenetics. Science 349, 647-650 (2015).

21. Gross, C. T. \& Canteras, N. S. The many paths to fear. Nat Rev Neurosci $13,651-658$ (2012).

22. Gunaydin, L. A. et al. Natural neural projection dynamics underlying social behavior. Cell 157, 1535-1551 (2014).

23. Han, S., Soleiman, M. T., Soden, M. E., Zweifel, L. S. \& Palmiter, R. D. Elucidating an Affective Pain Circuit that Creates a Threat Memory. Cell 162, 363-374 (2015).

24. Huang, T. et al. Identifying the pathways required for coping behaviours associated with sustained pain. Nature 565, 86-90 (2019).

25. Isogai, Y. et al. Molecular organization of vomeronasal chemoreception. Nature 478, 241-245 (2011).

26. Kunwar, P. S. et al. Ventromedial hypothalamic neurons control a defensive emotion state. eLife 4 (2015).

27. Lee, $\mathrm{H}$. et al. Scalable control of mounting and attack by Esr1+ neurons in the ventromedial hypothalamus. Nature 509, 627-632 (2014).

28. Leroy, F. et al. A circuit from hippocampal CA2 to lateral septum disinhibits social aggression. Nature 564, 213-218 (2018).

29. Lin, D. et al. Functional identification of an aggression locus in the mouse hypothalamus. Nature 470, 221-226 (2011).

30. Madisen, L. et al. A robust and high-throughput Cre reporting and characterization system for the whole mouse brain. Nature neuroscience 13, 133-140 (2010).

31. Mendes-Gomes, J. et al. Defensive behaviors and brain regional activation changes in rats confronting a snake. Behav Brain Res 381, 112469 (2020).

32. Olivier, B., Olivier-Aardema, R. \& Wiepkema, P. R. Effect of anterior hypothalamic and mammillary area lesions on territorial aggressive behaviour in male rats. Behav Brain Res 9, 59-81 (1983).

33. Olson, W. et al. Sparse genetic tracing reveals regionally specific functional 
organization of mammalian nociceptors. eLife 6 (2017).

34. Papes, F., Logan, D. W. \& Stowers, L. The vomeronasal organ mediates interspecies defensive behaviors through detection of protein pheromone homologs. Cell 141, 692-703 (2010).

35. Park, S. G. et al. Medial preoptic circuit induces hunting-like actions to target objects and prey. Nature neuroscience 21, 364-372 (2018).

36. Paschoalin-Maurin, T. et al. The Rodent-versus-wild Snake Paradigm as a Model for Studying Anxiety- and Panic-like Behaviors: Face, Construct and Predictive Validities. Neuroscience 369, 336-349 (2018).

37.Paxinos, G. and Franklin, K.B.J. The Mouse Brain in Stereotaxic Coordinates Academic Press (2001)

38. Risold, P. Y., Canteras, N. S. \& Swanson, L. W. Organization of projections from the anterior hypothalamic nucleus: a Phaseolus vulgaris-leucoagglutinin study in the rat. J Comp Neurol 348, 1-40 (1994).

39. Salay, L. D., Ishiko, N. \& Huberman, A. D. A midline thalamic circuit determines reactions to visual threat. Nature 557, 183-189 (2018).

40. Shang, C. et al. Divergent midbrain circuits orchestrate escape and freezing responses to looming stimuli in mice. Nat Commun 9, 1232 (2018).

41. Shang, C. et al. BRAIN CIRCUITS. A parvalbumin-positive excitatory visual pathway to trigger fear responses in mice. Science 348, 1472-1477 (2015).

42. Silva, B. A. et al. Independent hypothalamic circuits for social and predator fear. Nature neuroscience 16, 1731-1733 (2013).

43. Tovote, P. et al. Midbrain circuits for defensive behaviour. Nature 534, 206-212 (2016).

44. Vong, L. et al. Leptin action on GABAergic neurons prevents obesity and reduces inhibitory tone to POMC neurons. Neuron 71, 142-154 (2011).

45. Wang, L., Chen, I. Z. \& Lin, D. Collateral pathways from the ventromedial hypothalamus mediate defensive behaviors. Neuron 85, 1344-1358 (2015).

46. Wang, Y. et al. Large-scale forward genetics screening identifies Trpa1 as a chemosensor for predator odor-evoked innate fear behaviors. Nat Commun 9, 2041, (2018).

47. Wei, P. et al. Processing of visually evoked innate fear by a non-canonical thalamic pathway. Nat Commun 6, 6756 (2015).

48. Wickersham, I. R., Finke, S., Conzelmann, K. K. \& Callaway, E. M. Retrograde neuronal tracing with a deletion-mutant rabies virus. Nature methods 4, 47-49 (2007).

49. Yang, T. et al. Social Control of Hypothalamus-Mediated Male Aggression. Neuron 95, 955-970 e954 (2017).

50. Yilmaz, M. \& Meister, M. Rapid innate defensive responses of mice to looming visual stimuli. Curr Biol 23, 2011-2015 (2013). 
A

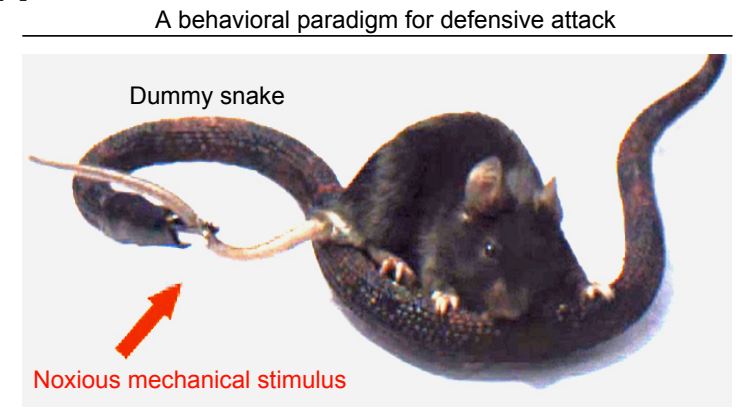

B

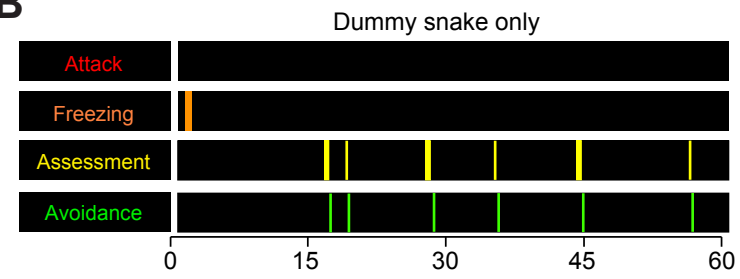

C

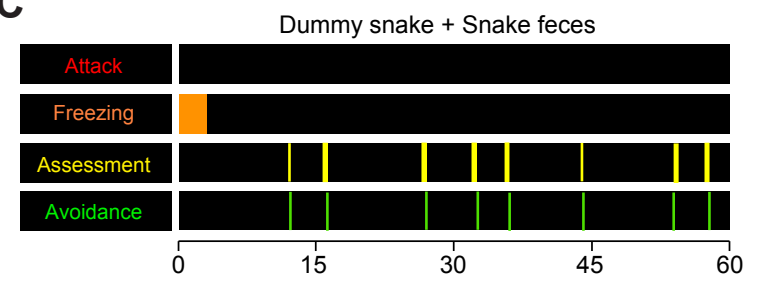

D

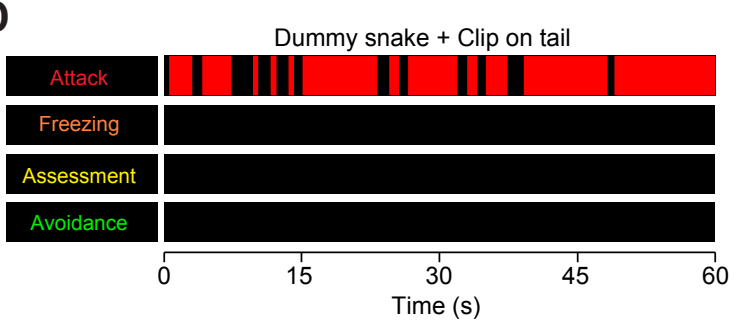

E

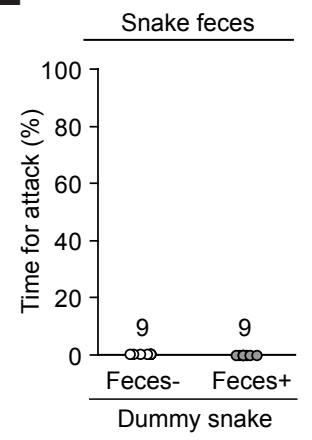

F

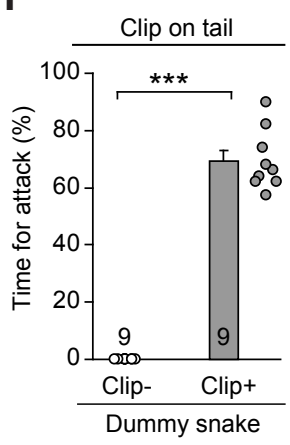

G
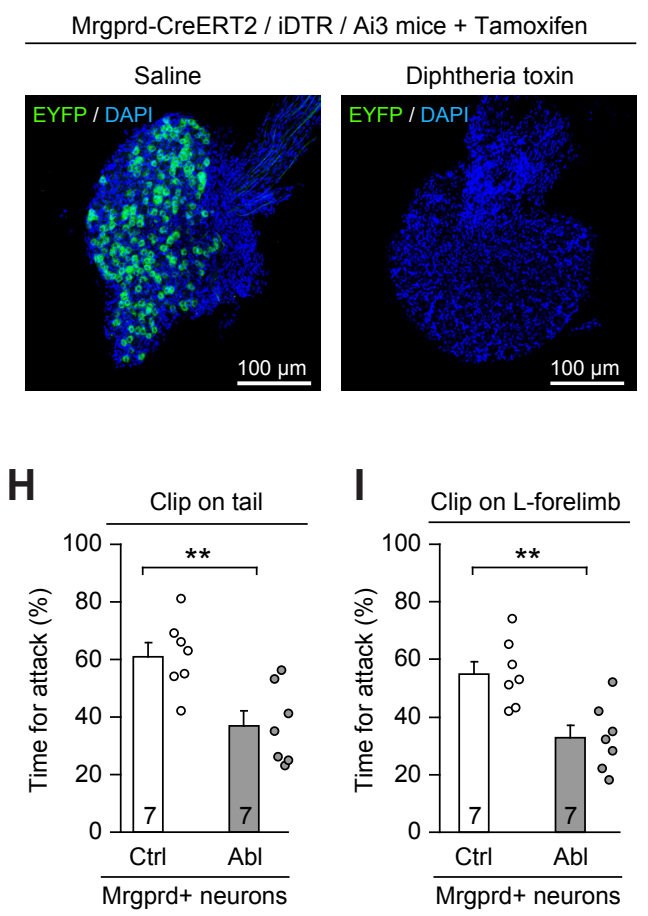

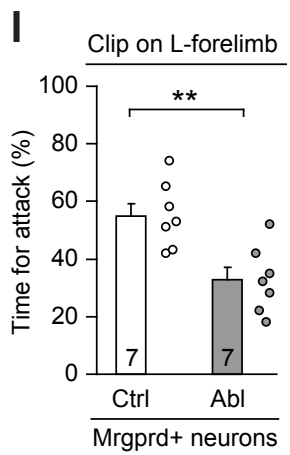

Figure 1 Xie et al., 2020 


\section{LEGENDS}

Figure 1 Noxious mechanical stimulus to evoke defensive attack in mice.

(A) An example picture showing a plastic dummy snake equipped with an alligator-clip to apply noxious mechanical stimulus (arrow) to mouse tail. (B-D) Behavioral ethograms of WT mice exposed to a dummy snake only (B), a dummy snake coated with snake feces (C), and a dummy snake equipped with an alligator-clip to apply noxious mechanical stimulus to mouse tail (D). The colored bars in the ethograms indicated the onset and offset of specific behaviors. (E) Time spent for attack by biting the dummy snake with and without snake feces. (F) Time spent for attack by biting the dummy snake with and without the alligator-clip to apply noxious mechanical stimulus. (G) Example micrographs of DRG of Mrgprd-CreERT2/iDTR/Ai3 mice treated with saline or Diphtheria toxin. $(\mathbf{H}, \mathbf{I})$ Time spent for attack in mice with and without ablation of putative Mrgprd+ DRG neurons evoked by noxious mechanical stimulus on tail $(\mathrm{H})$ or on left forelimb $(\mathrm{I})$. Number of mice was indicated in the graphs $(E, F, H, I)$. Data in $(E, F, H, I)$ are means \pm SEM (error bars). Statistical analyses in $(E, F, H, I)$ were performed by Student t-tests $\left({ }^{* *} \mathrm{P}<0.01\right.$; ${ }^{* *} \mathrm{P}<$ 0.001 ). For the $P$ values, see Table S4. 
A

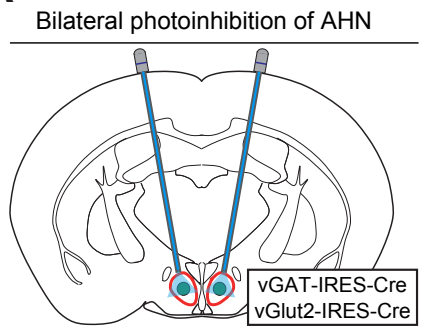

B

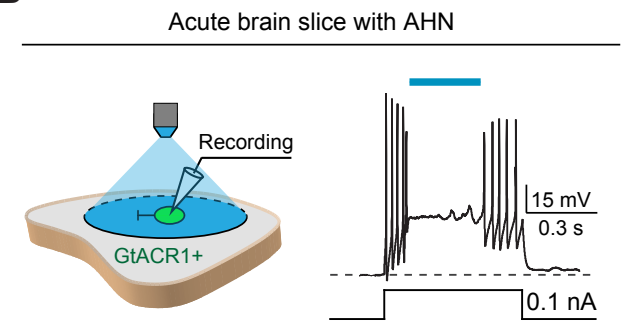

C

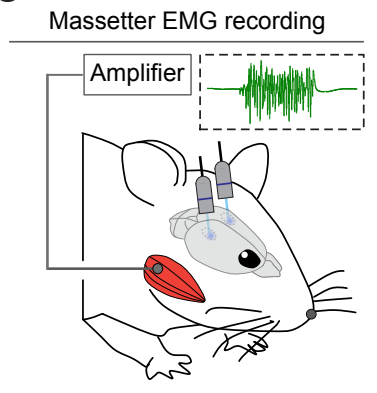

E vGAT-IRES-Cre ( $\mathrm{n}=7$ mice)

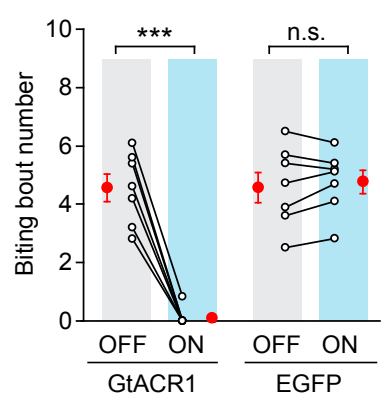

H

vGlut2-IRES-Cre ( $\mathrm{n}=7$ mice)

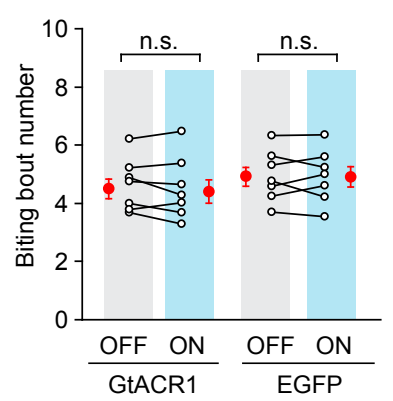

F
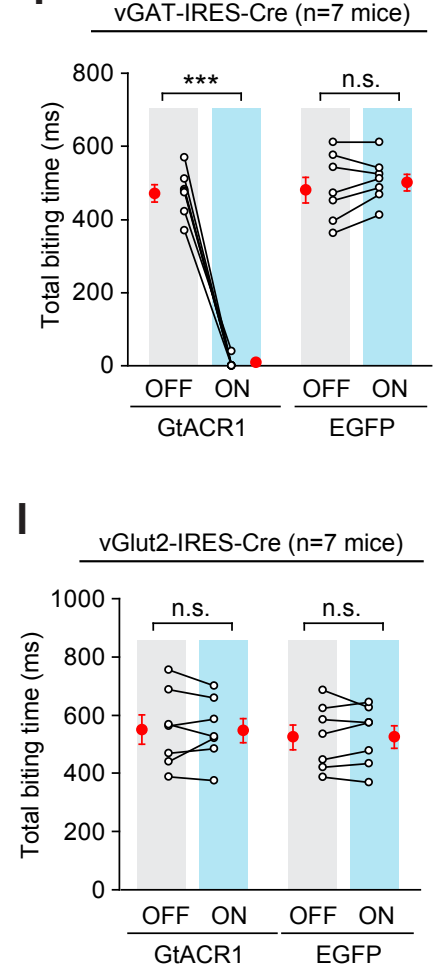

Figure 2 Xie et al., 2020 
Figure 2 AHN vGAT+ neurons are required for mechanically-evoked defensive attack.

(A) Schematic diagram of bilateral photoinhibition of AHN vGAT+ or vGlut2+ neurons. For the micrographs with the optic fiber tracks, see Figure S5A and S5B. For the analyses of cell-type specificity, see Figure S5C-S5F.

(B) Schematic diagram (left) and example trace (right) showing photoinhibition of AHN neurons expressing GtACR1. (C) Schematic diagram showing EMG recording from masseter muscles of mice. (D-F) Example traces (D) and quantitative analyses of biting bout number $(E)$ and total biting time $(F)$ within laser OFF phase and ON phase, showing the effect of photoinhibition of vGAT+ AHN neurons on mechanically-evoked biting attack. Mice with EGFP expressed in AHN vGAT+ neurons were used as a control for GtACR1. (G-I) Example traces (G) and quantitative analyses of biting bout number $(\mathrm{H})$ and total biting time $(\mathrm{I})$ within phases of laser OFF and ON, showing the effect of photoinhibition of AHN vGlut2+ neurons on mechanically-evoked biting attack. Mice with EGFP expressed in AHN vGlut2+ neurons were used as a control for GtACR1. For the effect of photoinhibition of AHN vGAT+ neurons on locomotion speed of freely-moving mice, see Figure $\mathrm{S} 5 \mathrm{H}$. Number of mice was indicated in the graphs $(E, F, H, I)$. Data in (E, F, H, I) are means \pm SEM (error bars). Statistical analyses in $(E, F, H, I)$ were performed by Student t-tests (n.s. $P>0.1$, ${ }^{* * *} P<$ 0.001). For the $P$ values, see Table $S 4$. 
A

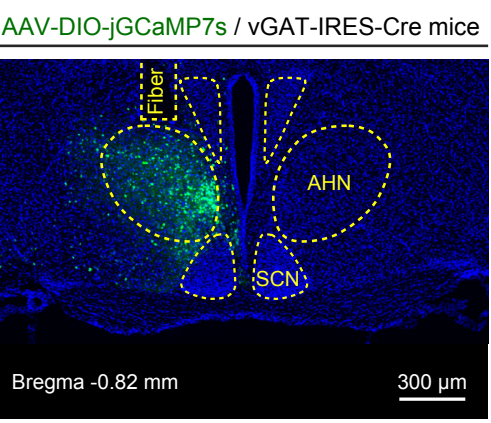

B

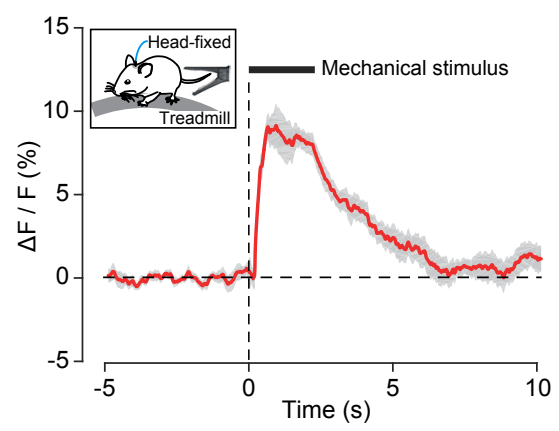

C

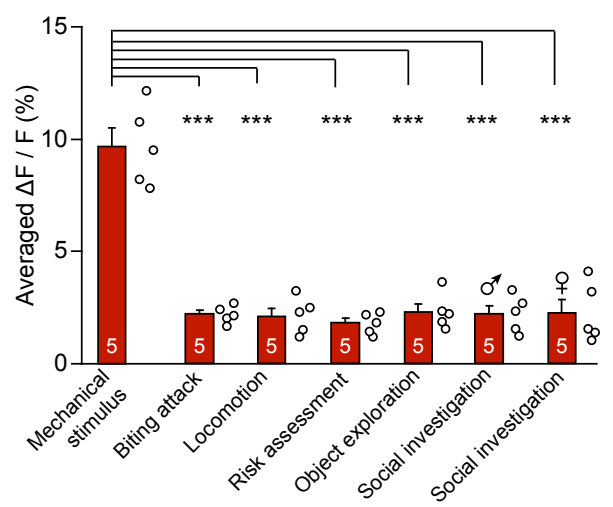

E

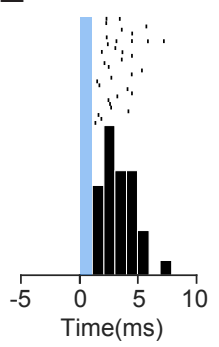

F
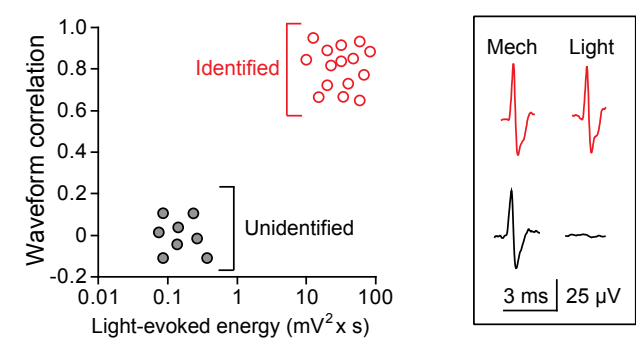
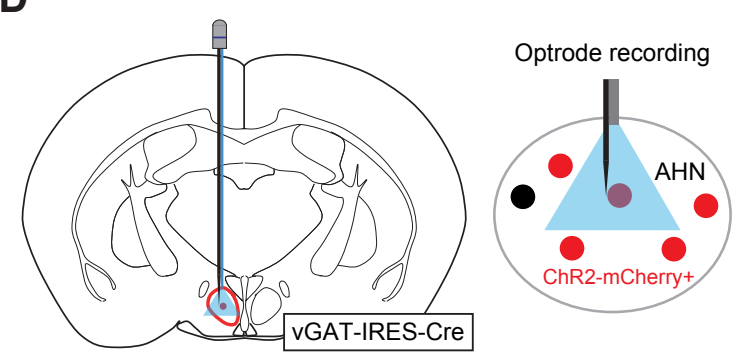

K

G
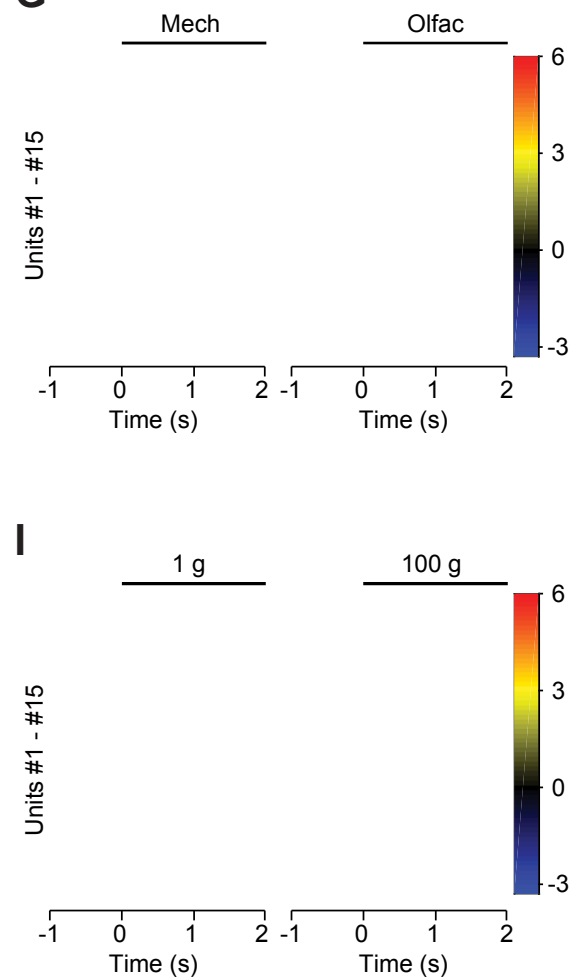

H

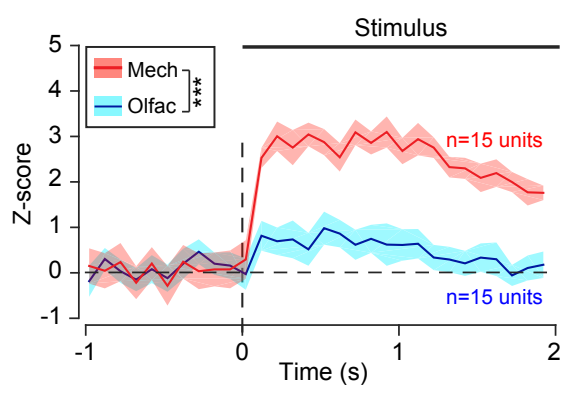

J

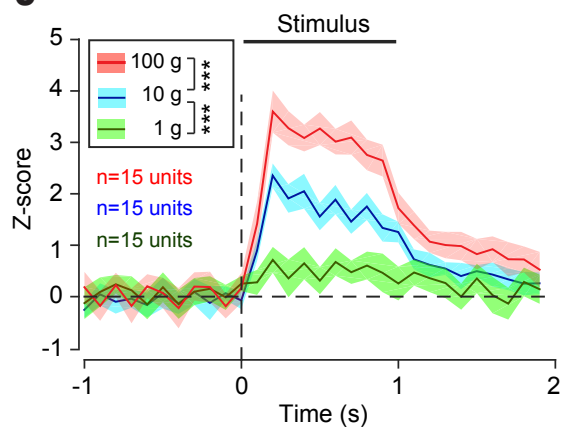

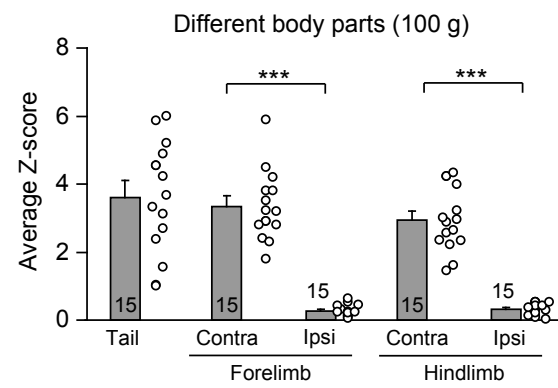

$\mathbf{L}$

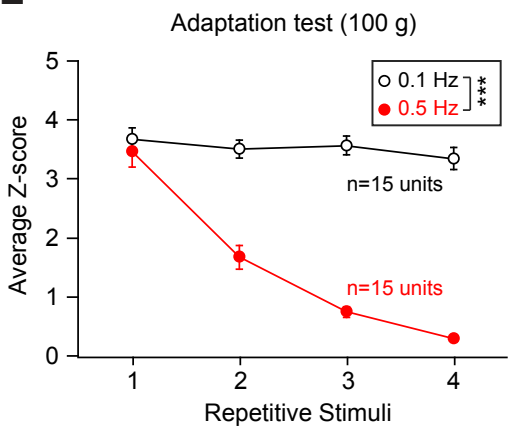

Figure 3 Xie et al., 2020 
Figure 3 AHN vGAT+ neurons encode mechanical stimuli.

(A) An example micrograph showing jGCaMP7s expressed in the AHN of vGAT-IRES-Cre mice and the optical fiber track above the AHN. For the analyses of cell-type specificity of jGCaMP7s expression, see Figure S6A and S6B. (B) Normalized GCaMP fluorescence changes $(\Delta F / F)$ in response to noxious mechanical stimulus on the tail. Inset, the head-fixed awake mouse standing on a treadmill was applied with noxious mechanical stimulus by tail clamping with an alligator-clip. (C) Quantitative analyses of peak GCaMP responses of AHN vGAT+ neurons in seven behavioral tests. The example trace for the test of "Mechanical stimulus" was in Figure 3B, while the other six example traces were in Figure S6C-S6H. (D) Schematic diagram of optrode recording from AHN vGAT+ neurons expressing ChR2-mCherry. For an example micrograph showing the optical fiber track and electrolytic lesion of recording site in the AHN, see Figure S7A. The principal component analysis for spike sorting of an example unit was in Figure S7B. (E) Raster and peri-stimulus time histogram (PSTH) of an example putative AHN vGAT+ neuron with spiking latency less than $5 \mathrm{~ms}$ relative to the onset of light pulses. (F) Correlation analysis of action potentials of individual units evoked either by light pulses (Light) or by mechanical stimuli (Mech), confirming a segregation between optogenetically identified units (Identified, red) and unidentified units (Unidentified, grey). (G) Heat-map PSTH of Z-scored firing rates of individual AHN vGAT+ neurons to mechanical stimuli (Mech) or olfactory stimuli (Olfac). For the schematic diagrams showing the application of mechanical stimuli and olfactory stimuli to the test mice, see Figure S7C and S7E. For the example units of putative AHN vGAT+ neurons in response to mechanical stimuli and olfactory stimuli, see Figure S7D and S7F. (H) Average PSTH of Z-scored firing rates of all identified AHN vGAT+ neurons to mechanical stimuli (Mech, red) and olfactory stimuli (Olfac, blue). (I) Heat-map PSTH of Z-scored firing rates of individual AHN vGAT+ neurons to mechanical stimuli applied with von Frey filaments $(1 \mathrm{~g}$, $100 \mathrm{~g}$ ). For the schematic diagram showing the application of von Frey filaments to the test mice, see Figure S7G. For the heat-map PSTH of Z-scored firing rates of individual AHN vGAT+ neurons to $10 \mathrm{~g}$ mechanical stimuli, see Figure S7H. (J) Average PSTH of Z-scored firing rates of all identified AHN VGAT+ neurons to mechanical force with different intensities. (K) Averaged peak Z-scored firing rates of all identified AHN vGAT+ neurons to mechanical stimuli (100 g) on different body parts. (L) Averaged peak Z-scored firing rates of all identified AHN vGAT+ neurons to four repetitive mechanical stimuli (100 g) applied at $0.1 \mathrm{~Hz}$ and $0.5 \mathrm{~Hz}$ on the tail. Number of mice (C) and number of units $(\mathrm{H}, \mathrm{J}, \mathrm{K}, \mathrm{L})$ were indicated in the graphs. Data in $(\mathrm{B}, \mathrm{C}, \mathrm{H}, \mathrm{J}, \mathrm{K}, \mathrm{L})$ are means \pm SEM (error bars). Statistical analyses were performed by Student t-tests $(C, K)$ and One-Way ANOVA $(H, J, L)\left({ }^{* * *} P<0.001\right)$. For the $P$ values, see Table $S 4$. 
A

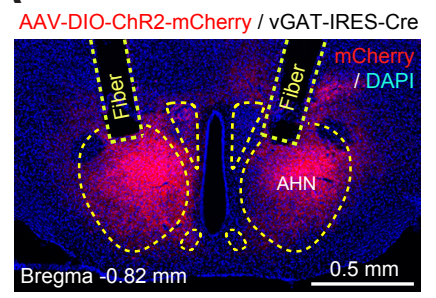

B

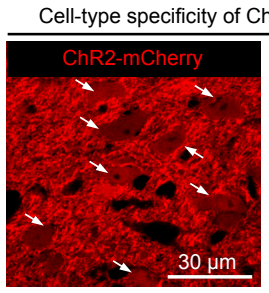

C

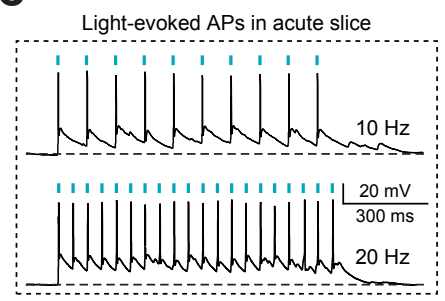

D

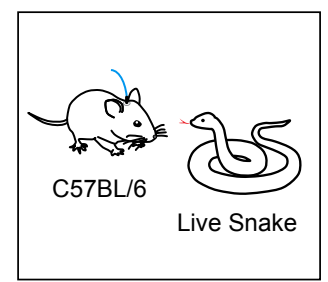

H

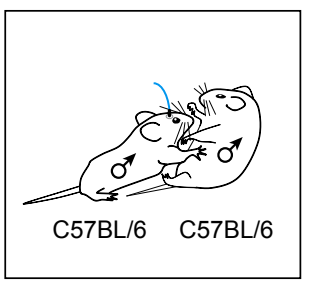

L

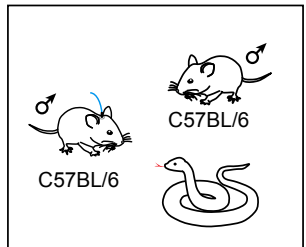

P

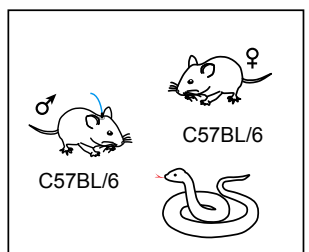

E

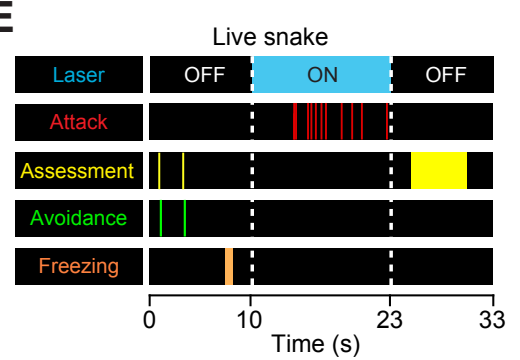

I

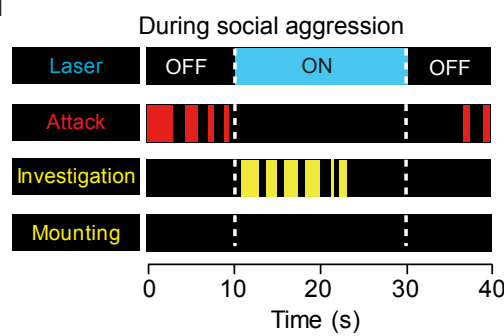

M

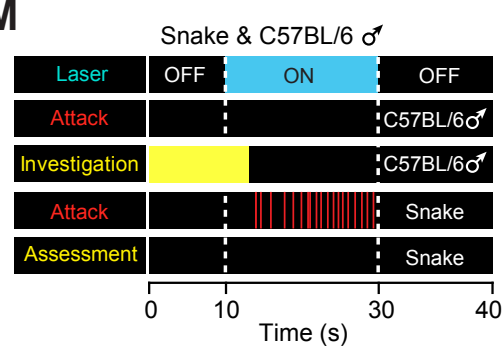

Q

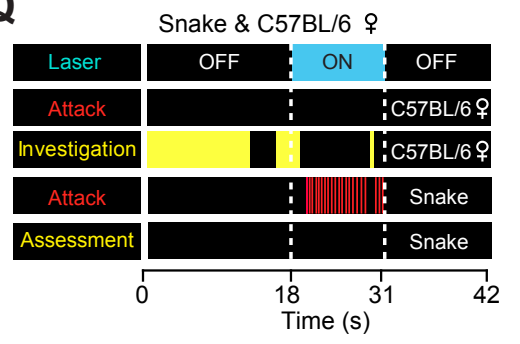

F
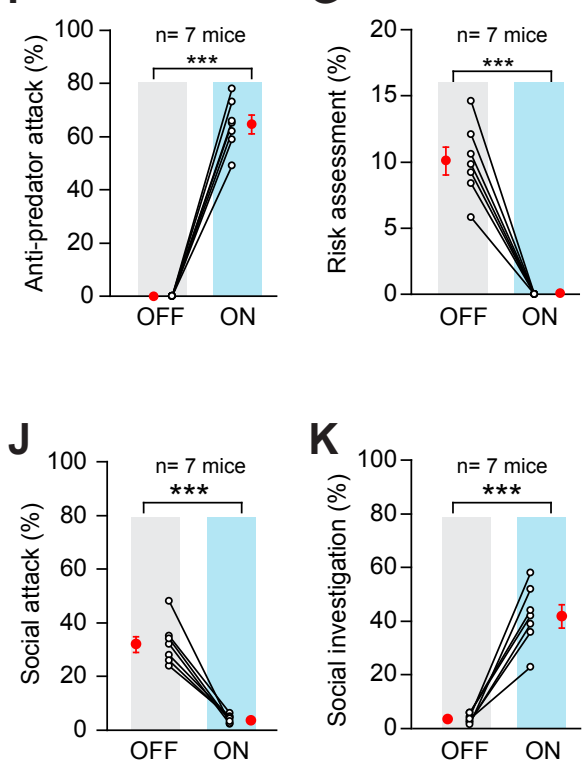

$\mathbf{N}$

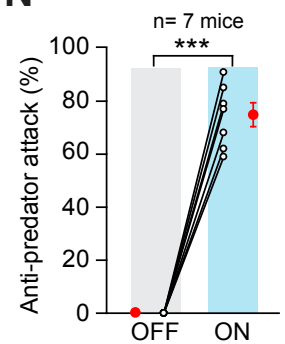

0

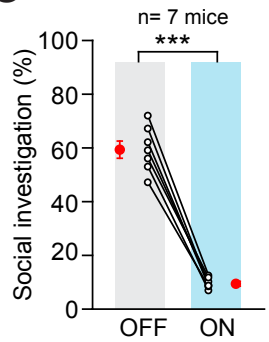

R

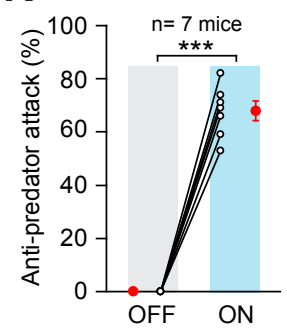

S

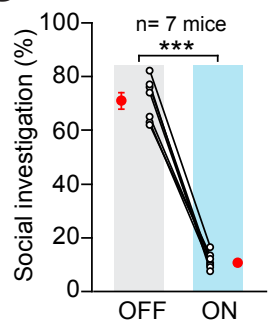

Figure 4 Xie et al., 2020 
Figure 4 Activation of AHN vGAT+ neurons trigger biting attack to non-social targets.

(A) An example coronal section showing ChR2-mCherry expression in the AHN of vGAT-IRES-Cre mice and bilateral optical fiber tracks above the AHN. (B) Example micrographs showing the cell-type specificity of ChR2-mCherry expression in GABA+ AHN neurons. Arrows indicated soma of GABA+ cells expressing ChR2-mCherry. For the quantitative analysis, see Figure S9A. (C) Light-pulse trains $(473 \mathrm{~nm}, 2 \mathrm{~ms}, 10 \mathrm{~mW}$, $10 \mathrm{~Hz}$ or $20 \mathrm{~Hz}$ ) reliably evoked phase-locked spiking activity in ChR2-mCherry+ AHN cells. (D) Schematic diagram showing a test mouse confronted with a live snake in the arena. (E-G) Example behavioral ethogram (E), quantitative analyses of time for anti-predator attack $(F)$ and time for risk assessment $(G)$ of mice before (OFF) and during (ON) photostimulation of AHN vGAT+ neurons. For quantitative analyses of time for freezing and avoidance, see Figure S9B and S9C. The colored bars in the ethograms indicated the onset and offset of specific behaviors. (H) Schematic diagram showing a test mouse exhibiting social aggression toward a male intruder. (I-K) Example behavioral ethogram (I), quantitative analyses of time for social attack $(\mathrm{J})$ and time for social investigation (K) of mice before (OFF) and during (ON) photostimulation of AHN vGAT+ neurons. For more behavioral analyses, see Figure S10. (L) Schematic diagram showing a test mouse confronted with a live snake and a male C57BL/6 mouse in the arena. (M-O) Example behavioral ethogram (M), quantitative analyses of time for anti-predator attack $(\mathrm{N})$ and time for social investigation (O) of test mice before (OFF) and during (ON) photostimulation of AHN vGAT+ neurons. For more behavioral analyses, see Figure S11 A-S11D. (P) Schematic diagram showing a test mouse confronted with a live snake and a female C57BL/6 mouse in the arena. (Q-S) Example behavioral ethogram (Q), quantitative analyses of time for anti-predator attack $(R)$ and time for social investigation (S) of test mice before (OFF) and during (ON) photostimulation of AHN vGAT+ neurons. For more behavioral analyses, see Figure S11E-S11H. Number of mice was indicated in the graphs $(F, G, J, K, N, O, R, S)$. Data in $(F, G, J, K, N, O, R, S$ ) are means $\pm S E M$ (error bars). Statistical analyses in ( $F, G, J, K, N, O, R, S$ ) were performed by Student t-tests ( ${ }^{* * *}$ $P<0.001)$. For the $P$ values, see Table $S 4$. 
A

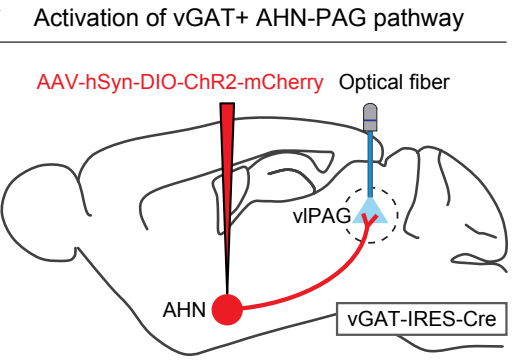

D

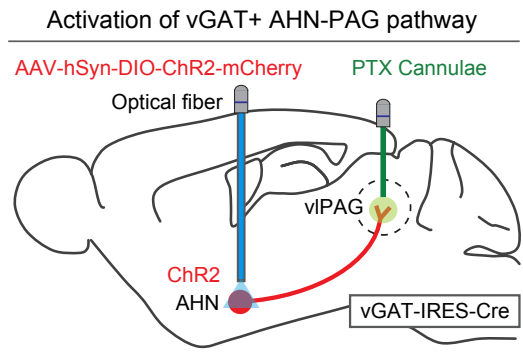

B

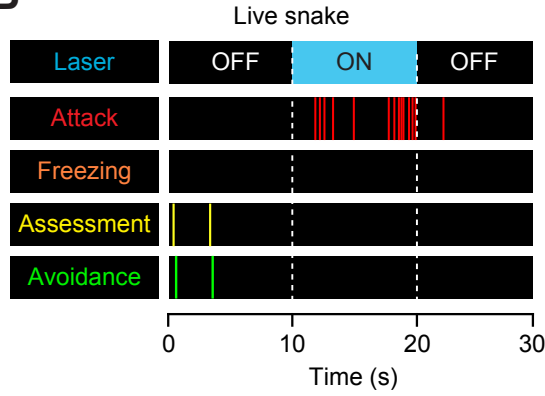

C

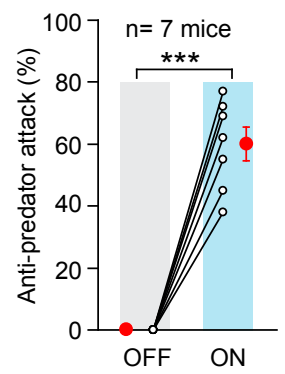

E

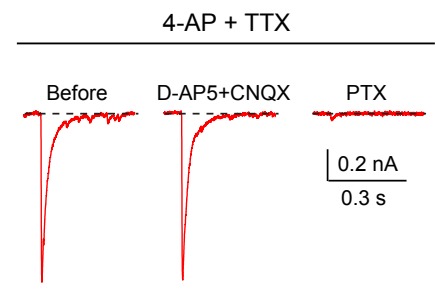

F

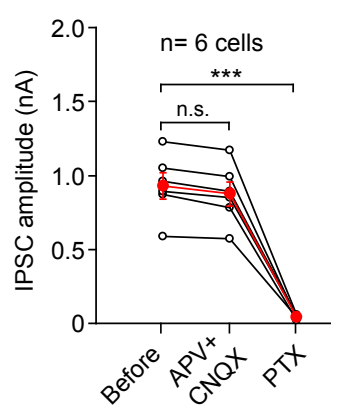

G

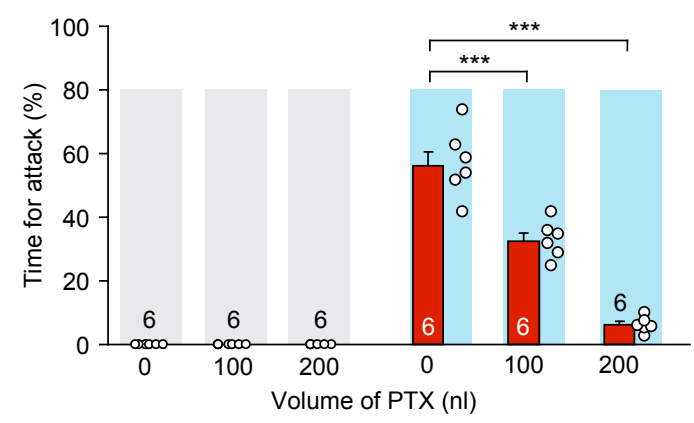

H

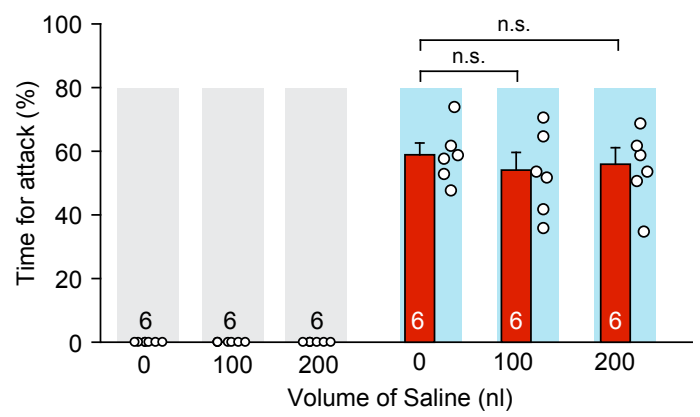

I

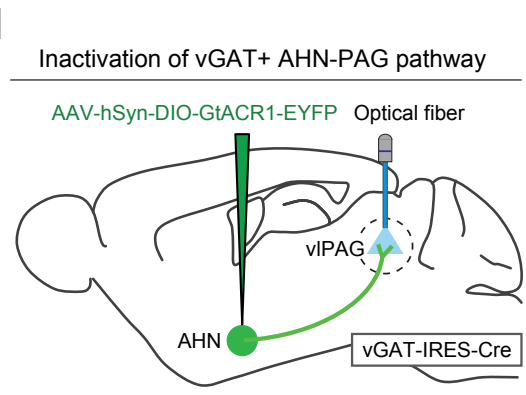

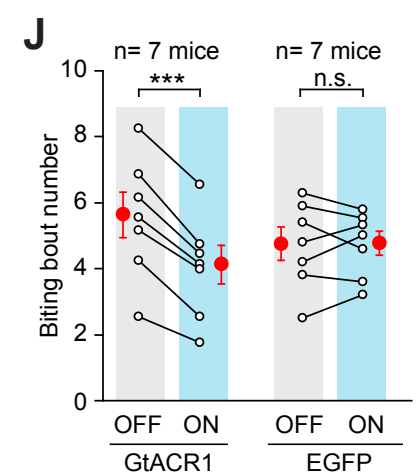

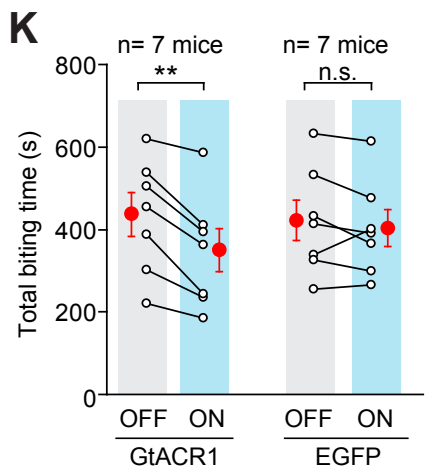


Figure 5 Role of vGAT+ AHN-PAG pathway for mechanically-evoked defensive attack.

(A) Schematic diagram showing AAV injection and optic fiber implantation for light stimulation of vGAT+ AHN-vIPAG pathway. For the example micrographs of the AHN and VIPAG, see Figure S13, A and B. (B, C) Example behavioral ethogram (B) and quantitative analysis of time for anti-predator attack (C) of mice before (OFF) and during (ON) activation of vGAT+ AHN-PAG pathway. For more behavioral analyses, see Figure S13C-S13E. For the analyses of VGAT+ AHN-LS pathway, see Figure S14. The colored bars in the ethograms indicated the onset and offset of specific behaviors. (D) Schematic diagram showing AAV injection into the AHN, optical fiber implantation above the AHN, and cannulae implantation above the VIPAG in VGAT-IRES-Cre mice. For the example micrographs with the optic fiber track and cannulae track above the AHN and vIPAG, see Figure S15A and S15B. (E, F) Example traces of postsynaptic currents (PSCs) recorded from vIPAG neurons $(E)$ and quantitative analyses of their amplitude $(F)$ showing the GABAergic action of VGAT+ AHN-PAG pathway is mediated by GABAa receptor. For the schematic diagram for slice physiology, see Figure S15C. (G, H) Quantitative analyses of light-evoked biting attack to live snake in mice with vIPAG treated with different doses of PTX $(G)$ or saline $(H)$. For the example behavioral ethograms, see Figure S15E and S15F. (I) Schematic diagram showing AAV injection and optic fiber implantation for photoinhibition of VGAT+ AHN-vIPAG pathway. For example micrographs of the AHN and vIPAG, see Figure S16A and S16B. (J, K) Quantitative analyses of biting bout number $(\mathrm{J})$ and total biting time $(K)$ toward dummy snake within phases of laser OFF and ON, showing the effect of photoinhibition of VGAT+ AHN-vIPAG pathway on mechanically-evoked biting attack. Mice with EGFP expressed in AHN vGAT+ neurons were used as a control for GtACR1. For example traces, see Figure S16C. Number of mice (C, G, H, J, K) and cells $(F)$ were indicated in the graphs. Data in $(C, F, G, H, J, K)$ are means $\pm S E M$ (error bars). Statistical analyses in $(C, F, G, H, J, K)$ were performed by Student t-tests (n.s. $P>0.1$; ** $\left.P<0.01 ;{ }^{* * *} P<0.001\right)$. For the $P$ values, see Table $S 4$. 


\section{METHODS}

\section{Animals}

All experimental procedures were conducted following protocols approved by the Administrative Panel on Laboratory Animal Care at the National Institute of Biological Sciences, Beijing (NIBS). The vGlut2-ires-Cre (Vong et al., 2011), vGAT-ires-Cre (Vong et al., 2011), Mrgprd-CreERT2 (Olson et al., 2017), Ai3 (Madisen et al., 2010) and Rosa26-iDTR (Buch et al., 2005) mouse lines were imported from the Jackson Laboratory (JAX Mice and Services). Mice were maintained on a circadian 12-h light/12-h dark cycle with food and water available ad libitum. Mice were housed in groups (3-5 animals per cage) before they were separated three days prior to virus injection. After virus injection, each mouse was housed in one cage for three weeks before subsequent experiments. To avoid potential sex-specific differences, we used male mice only.

The Wistar rats were purchased from Charles River Laboratories in China. The hamsters used in this study were offspring of wild greater long-tailed hamsters (Tscherskia triton) captured in northeast of China in 2005. The rats and hamsters were individually housed and maintained on a circadian 12-h light/12-h dark cycle with food and water available ad libitum.

The live snake (Elaphe schrenckii) used in this study were purchased from online pet stores (www.taobao.com). After arrival from shipment, the snakes were individually maintained in glass box $(35 \mathrm{~cm} \times 35 \mathrm{~cm} \times 35 \mathrm{~cm})$ with regular bedding at $24^{\circ} \mathrm{C}$. They were fed every twenty-four hours with rodents after euthanasia by $\mathrm{CO} 2$ inhalation. For mouse-versus-snake paradigm, one-month-old snake $(20-30 \mathrm{~g})$ was used. For rat-versus-snake and hamster-versus-snake paradigms, three-month-old snake (300-500 g) was used.

\section{AAV vectors}


The AAV serotype used in the present study is AAV2/9. The AAVs used in the present study are listed in Table S1. The plasmid for pAAV-EF1 $\alpha$-DIO-ChR2-mCherry (Addgene \#20297) was from Deisseroth Lab. The plasmid for pFUGW-hGtACR1-2A-EGFP (Addgene \#67795) was from Spudich Lab. The cDNA for AAV- EF1a-DIO-jGCaMP7s was from Kim Lab (Addgene \#104463). AAV-EF1a-DIO-SynaptoTag was from Thomas Südhof Lab at Stanford University. The viral particles were prepared by Taitool Inc. and BrainVTA Inc. The produced viral vector titers before dilution were in the range of $0.8-1.5 \times 10^{13}$ viral particles $/ \mathrm{ml}$. The final titer used for AAV injection is $5 \times 10^{12}$ viral particles $/ \mathrm{ml}$.

\section{Stereotaxic injection}

Mice were anesthetized with an intraperitoneal injection of tribromoethanol (125-250 $\mathrm{mg} / \mathrm{kg}$ ). Standard surgery was performed to expose the brain surface above the anterior hypothalamic nucleus (AHN). Coordinates used for AHN injection were: bregma -0.82 $\mathrm{mm}$, lateral $\pm 0.50 \mathrm{~mm}$, and dura $-4.40 \mathrm{~mm}$. The AAVs were stereotaxically injected with a glass pipette with $8^{\circ}$ angle from the lateral to medial. The injection was performed with the pipette connected to a Nano-liter Injector 201 (World Precision Instruments, Inc.) at a slow flow rate of $0.15 \mu \mathrm{l} / \mathrm{min}$ to avoid potential damage to local brain tissue. The pipette was withdrawn at least $20 \mathrm{~min}$ after viral injection. For fiber photometry experiments, AAV injections were unilateral and were followed by ipsilateral optical fiber implantation (see "Optical fiber implantation"). For optogenetic activation/inactivation experiments, AAV injections were bilateral and were followed by bilateral optical fiber implantation.

\section{Optical fiber implantation}

Thirty minutes after the AAV injection, a ceramic ferrule with an optical fiber $(230 \mu \mathrm{m}$ in diameter, N.A. 0.37) was implanted with the fiber tip on top of the AHN [unilateral: (bregma $-0.82 \mathrm{~mm}$, lateral $+0.4 \mathrm{~mm}$, dura $-5.00 \mathrm{~mm}$ ); bilateral: (bregma $-0.82 \mathrm{~mm}$, lateral $\pm 1.25 \mathrm{~mm}$, dura $-5.00 \mathrm{~mm}, 8^{\circ}$ angle from the lateral to medial)]. In some cases, the optical fiber was implanted with the fiber tip on top of the LS (bregma $0.56 \mathrm{~mm}$, lateral \pm 
$0.40 \mathrm{~mm}$, dura $-2.90 \mathrm{~mm}, 8^{\circ}$ angle from the lateral to medial) or vIPAG (bregma -4.30 $\mathrm{mm}$, lateral $\pm 0.70 \mathrm{~mm}$, dura $-1.95 \mathrm{~mm}, 18^{\circ}$ angle from the lateral to medial). The ferrule was then secured on the skull with dental cement. After implantation, the skin was sutured, and antibiotics were applied to the surgical wound. The optogenetic and fiber photometry experiments were conducted at least three weeks after optical fiber implantation. All experimental designs related to optical fiber implantation are summarized in Table S2.

For optogenetic manipulations, the output of the laser was measured and adjusted to 2, 5, $10 \mathrm{~mW}$ before each experiment. The pulse onset, duration, and frequency of light stimulation were controlled by a programmable pulse generator attached to the laser system. After AAV injection and fiber implantation, the mice were housed individually for three weeks before the behavioral tests.

\section{Preparation of the behavioral tests}

Before the behavioral tests, the animals were handled daily by the experimenters for at least three days. On the day of the behavioral test, the animals were transferred to the testing room and were habituated to the room conditions for $3 \mathrm{~h}$ before the experiments started. The apparatus was cleaned with $20 \%$ ethanol to eliminate odor cues from other animals. All behavioral tests were conducted during the same circadian period (13:00-19:00). All behaviors were scored by the experimenters, who were blind to the animal treatments.

\section{Rodent-versus-snake paradigm}

Mouse-versus-snake paradigm was performed in an enclosed box $(10 \mathrm{~cm} \times 10 \mathrm{~cm} \times$ $35 \mathrm{~cm}$ ) without bedding. The arena was cleaned with $20 \%$ ethanol to eliminate odor cues from other mice. The mouse $(20 \mathrm{~g}-30 \mathrm{~g})$ was habituated in the arena for 10 minutes. Then a young snake $(20 \mathrm{~g}-30 \mathrm{~g})$ was placed in the arena. Before predatory attack from the snake, the mice exhibited risk assessment and freezing. When the snake initiated predatory attack, the mice exhibited avoidance or jumping escape. When the snake 
gripped the body parts of the mice with the teeth, the mice usually defensively attacked the snake by biting (Movie S1). The trial ended when the mouse stopped defensive attack.

Rat-versus-snake paradigm and hamster-versus-snake paradigm were performed in an enclosed box $(25 \mathrm{~cm} \times 25 \mathrm{~cm} \times 35 \mathrm{~cm})$ without bedding. The arena was cleaned with $20 \%$ ethanol to eliminate odor cues from other animal. The rat $(400 \mathrm{~g}-500 \mathrm{~g})$ or the hamster (300 g- $400 \mathrm{~g}$ ) was habituated in the arena for 10 minutes. Then an adult snake $(300 \mathrm{~g}-500 \mathrm{~g})$ was placed in the arena. Before predatory attack from the snake, the rat or the hamster exhibited freezing, risk assessment and avoidance. When the snake initiated predatory attack, the rat or the hamster exhibited avoidance or jumping escape. When the snake gripped the body parts of the rat or hamster with the teeth, they usually defensively attacked the snake by biting (Movie S2 and S3). The trial ended when the rat or hamster stopped defensive attack.

\section{Measurement of defensive behaviors to dummy snake}

The plastic dummy snake was purchased from a merchant in Tao-Bao online store (www.taobao.com). The dummy snake $(30 \mathrm{~g})$ was either coated with fresh snake feces to provide olfactory cues of snake or equipped with a head-like alligator-clip $(3 \mathrm{~g})$ to provide noxious mechanical stimuli. The mechanical force from the alligator-clip, measured with spring dynamometer, was $\sim 366$ grams. The mechanical stimuli were applied either on the tail or on the four limbs of mice. In some experiments (Figure S2, G-I), the ambient light in the behavioral box was switched on (Light+, 50 lux) and off (Light-, $\sim 0.002$ lux) to measure the contribution of visual cues to mechanically-evoked defensive attack. In some experiments (Figure S3), neutral objects (wood block, plastic lid) were connected to alligator clip to examine whether mice exhibit defensive attack to neutral object in the presence of noxious mechanical stimuli.

The defensive responses of the mice to the dummy snake in the enclosed arena (25 $\mathrm{cm} \times 25 \mathrm{~cm} \times 35 \mathrm{~cm}$ ) were recorded with a high-speed camera (160 frames / s). The 
arena was cleaned with $20 \%$ ethanol to eliminate odor cues from other mice. The time for freezing, risk assessment, avoidance and biting attack were measured by visual inspection off-line and plotted with a behavioral ethogram (e.g. Figure 1, B-D).

\section{Genetic ablation of Mrgprd+ neurons}

To ablate Mrgprd+ neurons, a two-step strategy of drug injections was used. First, tamoxifen was injected intraperitoneally for eight consecutive days at a dosage of 75 $\mathrm{mg} / \mathrm{kg}$ in 14-day old Mrgprd-CreERT2/iDTR/Ai3 male mice. Tamoxifen injection at this dosage at this developmental stage was shown to induce Cre expression in Mrgprd-expressing DRG neurons with high specificity $(88.1 \pm 1 \%)$ and high efficiency $(92.9 \pm 4.6 \%)$ (Olsen et al., 2017). Second, three weeks after the last dose of tamoxifen injection, diphtheria toxin (DT) was injected intraperitoneally for three consecutive days at a dosage of $4 \mathrm{mg} / \mathrm{kg}$ in the same group of mice. The defensive attack behavior to the dummy snake was measured three weeks after the last dose of DT injection. The efficiency of genetic ablation of Mrgprd+ neurons were tested by immunostaining of EYFP in the dorsal root ganglions (DRG) at the lumbar and sacral segments.

\section{EMG electrode implantation and EMG recording}

To monitor jaw muscle activity, we implanted chronic EMG electrodes in the right masseter muscles of the jaw. The EMG electrode was made with flexible multi-strand stainless steel wires (A-M Systems, No. 793200). The insulation of a small segment of the wire $(\sim 0.5 \mathrm{~mm})$ was removed to expose the electrode to the muscle. During the surgical procedure, the wires were threaded through and anchored with a knot on the muscle. The wires were then threaded beneath the skin of mouse face and attached to the ground electrodes at the base of the skull with dental cement. After 3 day of recovery from surgery, mice were connected to flexible EMG connection cables and allowed to adapt for at least 1 day. The EMG signals were recorded using a Microelectrode AC Amplifier Model 1800 (A-M System), filtered (10-500 Hz EMG recordings) and digitized at $250 \mathrm{~Hz}$ using the software Spike2. A flashing LED triggered by a 1-s square-wave 
pulse was simultaneously recorded to synchronize the video and EMG signals. The effects of photoinhibition (Figure 2, D-I; Figure 5, I-K) and photostimulation (Figure S9D and S9E) of AHN VGAT+ neurons on defensive attack were examined in the present study.

\section{Measuring the effects of GtACR1-mediated photoinhibition on defensive attack}

To test the effects of photoinhibition of vGAT+ and vGlut2+ AHN neurons on mechanically-evoked defensive attack, we injected AAV-EF1a-DIO-GtACR1-2A-EGFP into the AHN of $v G A T$-IRES-Cre or vGlut2-IRES-Cre mice bilaterally, followed by optical fibers implanted above the injection sites bilaterally. Three weeks after AAV injection, the mice were subjected to the regular procedure to test mechanically-evoked defensive attack behavior. GtACR1-mediated photoinhibition was achieved by laser illumination (473 nm, 2s-OFF/2s-ON, $10 \mathrm{~mW}$ ) on GtACR1-expressing vGAT+ or vGlut2+ AHN neurons during mechanically-evoked defensive attack. The duration and bout number of mechanically-evoked biting toward the dummy snake, as read out by analyzing EMG traces (Figure S5G), were analyzed off-line. In a control experiment, the locomotion speed of freely-moving mice before and during photoinhibition of vGAT+ AHN neurons was measured with the video recorded from a camera above the mice.

To test the effects of photoinhibition of VGAT+ AHN-vIPAG pathway on mechanically-evoked defensive attack, we injected AAV-EF1a-DIO-GtACR1-2A-EGFP into the AHN of VGAT-IRES-Cre mice bilaterally, followed by optical fibers implanted above the vIPAG bilaterally. Six weeks after AAV injection, the mice were subjected to the regular procedure to test mechanically-evoked defensive attack behavior. GtACR1-mediated photoinhibition was achieved by laser illumination (473 nm, 2s-OFF/2s-ON, $10 \mathrm{~mW}$ ) on GtACR1-expressing axon terminals of VGAT+ AHN neurons during mechanically-evoked defensive attack. The duration and bout number of mechanically-evoked biting toward the dummy snake, as read out by analyzing EMG traces (Figure S5G), were analyzed off-line. 


\section{Fiber photometry recording}

A fiber photometry system (ThinkerTech, Nanjing, China) was used for recording GCaMP signals from genetically identified neurons (Gunaydin et al., 2014). To induce fluorescence signals, a laser beam from a laser tube $(488 \mathrm{~nm})$ was reflected by a dichroic mirror, focused by a $10 \times$ lens (N.A. 0.3) and coupled to an optical commutator. A 2-m optical fiber $(230 \mu \mathrm{m}$ in diameter, N.A. 0.37$)$ guided the light between the commutator and implanted optical fiber. To minimize photo bleaching, the power intensity at the fiber tip was adjusted to $0.02 \mathrm{~mW}$. The jGCaMP7s (Dana et al., 2019) fluorescence was band-pass filtered (MF525-39, Thorlabs) and collected by a photomultiplier tube (R3896, Hamamatsu). An amplifier (C7319, Hamamatsu) was used to convert the photomultiplier tube current output to voltage signals, which were further filtered through a low-pass filter ( $40 \mathrm{~Hz}$ cut-off; Brownlee 440$)$. The analogue voltage signals were digitalized at $100 \mathrm{~Hz}$ and recorded by a Power 1401 digitizer and Spike2 software (CED, Cambridge, UK).

AAV-hSyn-DIO-jGCaMP7s was stereotaxically injected into the AHN of VGAT-ires-Cre mice followed by optical fiber implantation above the AHN (see "Stereotaxic injection" and "Optical fiber implantation"). Three weeks after AAV injection, fiber photometry was used to record GCaMP signals from the cell bodies of VGAT+ AHN neurons in seven different behavioral tests (see below). A flashing LED triggered by a 1-s square-wave pulse was simultaneously recorded to synchronize the video and GCaMP signals. After the experiments, the optical fiber tip sites above the vGAT+ AHN neurons were histologically examined in each mouse.

\section{Measuring GCaMP signals before and during mechanical stimuli}

Head-fixed awake mice with the optical fiber connected to the fiber photometry system were allowed to stand on a circular treadmill. Then noxious mechanical stimuli were applied by the alligator clip to mouse tail while the GCaMP fluorescence was recorded. The GCaMP signals were measured by normalizing GCaMP fluorescence $(\Delta F / F)$ and 
aligned with the initiation of mechanical stimuli.

\section{Measuring GCaMP signals before and during mechanically-evoked biting}

Mice with the optical fiber connected to the fiber photometry system freely explored the arena for $10 \mathrm{~min}$. Then the mouse tail was clamped by the alligator-clip connected to the dummy snake. The GCaMP fluorescence and mechanically-evoked biting-like attack were simultaneously recorded. The GCaMP signals were measured by normalizing GCaMP fluorescence $(\Delta F / F)$ and aligned with the initiation of biting-like attack.

\section{Measuring GCaMP signals before and during locomotion}

Head-fixed awake mice with the optical fiber connected to the fiber photometry system were allowed to stand on a circular treadmill. The spontaneous locomotion and the GCaMP fluorescence were simultaneously recorded. The GCaMP signals were measured by normalizing GCaMP fluorescence $(\Delta F / F)$ and aligned with the initiation of locomotion.

\section{Measuring GCaMP signals before and during risk assessment}

Mice with optical fibers connected to the fiber photometry system freely explored the arena for $10 \mathrm{~min}$. Then a live snake was introduced to the arena while the GCaMP signals and risk assessment were simultaneously recorded. The GCaMP signals were measured by normalizing GCaMP fluorescence $(\Delta F / F)$ and aligned with the initiation of risk assessment.

\section{Measuring GCaMP signals before and during object exploration}

Mice with optical fibers connected to the fiber photometry system freely explored the arena for $10 \mathrm{~min}$. Then a wood block $(3 \mathrm{~cm} \times 3 \mathrm{~cm} \times 3 \mathrm{~cm})$ was introduced to the arena while the GCaMP signals and object exploration were simultaneously recorded. The GCaMP signals were measured by normalizing GCaMP fluorescence $(\Delta F / F)$ and aligned with the initiation of object exploration.

Measuring GCaMP signals before and during social investigation 
Mice with optical fibers connected to the fiber photometry system freely explored the arena for $10 \mathrm{~min}$. Then a male or female C57BL/6 mouse was introduced to the arena while the GCaMP signals and social investigation were simultaneously recorded. The GCaMP signals were measured by normalizing GCaMP fluorescence $(\Delta F / F)$ and aligned with the initiation of social investigation.

\section{Single-unit recording with optrode}

An optrode was used to identify the single-unit activity of vGAT+ AHN neurons. AAV2/9-hSyn-DIO-ChR2-mCherry was injected into the AHN of vGAT-ires-Cre mice. Three weeks after viral injection, single-unit recording was performed with an optrode in the AHN of head-fixed awake mouse standing on the treadmill. The single-channel optrode was made by assembling an optic fiber $(230 \mu \mathrm{m})$ parallel with a glass-coated tungsten electrode (1-3 M $\Omega$ ). The distance between the two tips was $\sim 200 \mu \mathrm{m}$. The optrode was vertically advanced into the AHN with a Narishige micro-manipulator so that the tungsten electrode tip was in the AHN while the optic fiber was above the AHN, which minimized damage to the AHN by the optic fiber (Figure S7A). The spikes were amplified by a differential amplifier (Model 1800, A-M Systems, Everett, WA, USA), digitized $(10 \mathrm{kHz})$ and stored by Spike2 software (Version 7.03$)$. When the spikes from mechanically-responsive units were isolated, a train $(10 \mathrm{~Hz}, 1 \mathrm{sec})$ of light stimulations (1 ms) was delivered to test if the units were from ChR2-expressing neurons, which are presumably vGAT+. The spikes from putative vGAT+ neurons had to conform to two criteria: first, their latency to the light pulse should be less than $5 \mathrm{~ms}$; second, their waveform should be similar to that of spikes evoked by sensory stimulation. Only units with spikes faithfully following the light stimulations with latency less than $5 \mathrm{~ms}$ were further tested for sensory-evoked responses. The spike sorting was performed with Spike2 Software (Version 7.03) in accordance with our previous work (Shang et al., 2015). For a certain train of action potential, after setting the threshold of the spikes, Spike2 automatically generated the templates and performed the spike-sorting. The 
quality of spike clustering was further confirmed by principal component analysis (Figure S7B). During single-unit recording, the application of mechanical and olfactory stimuli by the experimenter was recorded by a video camera. A flashing LED triggered by a 1-s square-wave pulse was simultaneously recorded to synchronize the video and single-unit recording.

\section{Mechanical and olfactory stimuli}

When the single-unit activity of putative vGAT+ AHN units was isolated, we applied mechanical or olfactory stimuli to the test mice. Mechanical stimuli were applied either by alligator-clip (Figure S7C) or by Von Frey Filaments (Figure S7G). The alligator-clip that clamped mouse tail generated mechanical force ( 366 grams), which was measured by two spring dynamometers connected to the two jaws of alligator-clip directing toward the opposite directions. Three Von Frey Filaments with graded mechanical force $(1 \mathrm{~g}, 10 \mathrm{~g}$, and $100 \mathrm{~g}$ ) were used to poke different body parts of mice. To examine whether AHN vGAT+ neurons also respond to mechanical stimuli applied on other parts of the body, we used Von Frey filament $(100 \mathrm{~g})$ to poke four limbs of the mouse. To examine adaptation of AHN vGAT+ neurons to repetitive mechanical stimuli, Von Frey filament $(100 \mathrm{~g})$ were used to poke the tail of the test mice four times at a certain frequency $(0.1$ $\mathrm{Hz}$ or $0.5 \mathrm{~Hz}$ ). To mimic olfactory cues of predator, fresh snake feces were coated on a cotton swab and presented to the test mice with a distance of $2 \mathrm{~cm}$ between cotton swab and the nose tip (Figure S7E).

\section{Verification of recording sites}

The recording sites of the putative vGAT+AHN neurons were marked with electrolytic lesions applied by passing positive currents $(40 \mu \mathrm{A}, 10 \mathrm{~s})$ through the tungsten electrode. Under deep anesthesia with urethane, the brain was perfused with saline and PBS containing 4\% PFA. After regular histological procedure, frozen sections were cut at 40 $\mu \mathrm{m}$ in thickness and counterstained with DAPI for histological verification of recording sites (Figure S7A). 


\section{Cell-type-specific RV tracing}

The modified rabies virus based three-virus system was used for mapping the whole-brain inputs to vGAT+ AHN neurons (Wickersham et al., 2007). All the viruses included AAV2/9-CAG-DIO-EGFP-2A-TVA (5 $\quad x \quad 10^{12}$ viral particles $\left./ \mathrm{ml}\right)$, AAV2/9-CAG-DIO-RG (5 × $10^{12}$ viral particles/ml), and EnvA-pseudotyped, glycoprotein (RG)-deleted and DsRed-expressing rabies virus (RV-EvnA-DsRed, RV) $\left(5.0 \times 10^{8}\right.$ viral particles $/ \mathrm{ml}$ ), which were packaged and provided by BrainVTA Inc. (Wuhan, China). A mixture of AAV2/9-CAG-DIO-EGFP-2A-TVA and AAV2/9-CAG-DIO-RG (1:1, $200 \mathrm{nl})$ was stereotaxically injected into the AHN of vGAT-ires-Cre mice unilaterally. Two weeks after AAV helper injection, RV-EvnA-DsRed (300 nl) was injected into the same location in the AHN of vGAT-ires-Cre mice in a biosafety level-2 lab facility. Starter neurons were characterized by the coexpression of DsRed and EGFP, which were restricted in the AHN (Figure S6C).

One week after injection of rabies virus, mice were perfused with saline followed by 4\% paraformaldehyde (PFA) in PBS. After $8 \mathrm{~h}$ of post-fixation in 4\% PFA, coronal brain sections at $40 \mu \mathrm{m}$ in thickness were prepared using a cryostat (Leica CM1900). All coronal sections were collected and stained with DAPI. The coronal brain sections were imaged with an Olympus VS120 epifluorescence microscope (10x objective) and analyzed with ImageJ. For quantifications of subregions, boundaries were based on the mouse brain atlas (Paxinos and Franklin, 2001). We selectively analyzed the retrogradely labeled dense areas. The factional distribution of total cells labeled by rabies virus was measured (Figure S8F).

Cell-counting strategies

Cell-counting strategies are summarized in Table S3. For counting cells in the AHN, we collected coronal sections $(40 \mu \mathrm{m})$ from bregma $-0.34 \mathrm{~mm}$ to bregma $-1.34 \mathrm{~mm}$ for each mouse. The outline of the AHN was according to the mouse brain atlas (Paxinos and Franklin, 2001). We acquired confocal images (20x objective, Zeiss LSM 780) 
followed by cell counting with ImageJ software. By combining fluorescent in situ hybridization and immunohistochemistry, we counted the number of VGAT+ and vGlut2+ cells in the AHN (Figure S4, A-C) and calculated the percentages of VGAT+ and vGlut2+ neurons in the neuronal population labeled by EGFP (Figure S4, D-O). With immunohistochemical staining of glutamate and GABA, we calculated the percentages of glutamate ${ }^{+}$and $\mathrm{GABA}^{+}$neurons in the neuronal population labeled by EGFP (Figure S5, C-F), GCaMP7 (Figure S6, A \& B), or mCherry (Figure S9, A \& B).

To analyze monosynaptic inputs of VGAT+ AHN neurons, we counted DsRed+ cells in a series of brain areas (Figure S8, E \& F). For counting cells in the LS, we collected coronal sections $(40 \mu \mathrm{m})$ from bregma $+1.54 \mathrm{~mm}$ to bregma $-0.10 \mathrm{~mm}$. For counting cells in the MPA, we collected coronal sections $(40 \mu \mathrm{m})$ from bregma $+0.74 \mathrm{~mm}$ to bregma $-0.58 \mathrm{~mm}$ for each mouse. For counting cells in the PVH, we collected coronal sections $(40 \mu \mathrm{m})$ from bregma $-0.58 \mathrm{~mm}$ to bregma $-1.22 \mathrm{~mm}$. For counting cells in the SO, we collected coronal sections $(40 \mu \mathrm{m})$ from bregma $-0.58 \mathrm{~mm}$ to bregma $-0.94 \mathrm{~mm}$. For counting cells in the $\mathrm{VMH}$, we collected coronal sections $(40 \mu \mathrm{m})$ from bregma $-1.06 \mathrm{~mm}$ to bregma $-2.06 \mathrm{~mm}$. For counting cells in the DM, we collected coronal sections $(40 \mu \mathrm{m})$ from bregma $-1.34 \mathrm{~mm}$ to bregma $-2.18 \mathrm{~mm}$. For counting cells in the MA, we collected coronal sections $(40 \mu \mathrm{m})$ from bregma $-0.94 \mathrm{~mm}$ to bregma $-2.18 \mathrm{~mm}$. For counting cells in the PVT, we collected coronal sections $(40 \mu \mathrm{m})$ from bregma $-0.22 \mathrm{~mm}$ to bregma $-2.18 \mathrm{~mm}$. For counting cells in the PMD, we collected coronal sections $(40 \mu \mathrm{m})$ from bregma $-2.46 \mathrm{~mm}$ to bregma $-2.70 \mathrm{~mm}$. For counting cells in the PMV, we collected coronal sections $(40 \mu \mathrm{m})$ from bregma $-2.30 \mathrm{~mm}$ to bregma $-2.54 \mathrm{~mm}$. For counting cells in the $\mathrm{PH}$, we collected coronal sections $(40 \mu \mathrm{m})$ from bregma $-1.82 \mathrm{~mm}$ to bregma -2.70 $\mathrm{mm}$. For counting cells in the $\mathrm{S}$, we collected coronal sections $(40 \mu \mathrm{m})$ from bregma $-2.46 \mathrm{~mm}$ to bregma $-4.36 \mathrm{~mm}$. For counting cells in the $\mathrm{AHi}$, we collected coronal sections $(40 \mu \mathrm{m})$ from bregma $-1.94 \mathrm{~mm}$ to bregma $-3.80 \mathrm{~mm}$. For counting cells in the LPB, we collected coronal sections $(40 \mu \mathrm{m})$ from bregma $-4.96 \mathrm{~mm}$ to bregma $-5.68 \mathrm{~mm}$. 
The outlines of these brain areas were according to the mouse brain atlas (Paxinos and Franklin, 2001). We acquired fluorescent images (10x objective, Olympus) followed by cell counting with ImageJ software.

\section{ChR2-mediated photostimulation of VGAT+ AHN neurons}

AAV-hSyn-DIO-ChR2-mCherry was bilaterally injected into the AHN of VGAT-IRES-Cre mice, followed by optical fibers implanted bilaterally above the injection sites. Three weeks after AAV injection, the VGAT+ AHN neurons were photostimulated ( $473 \mathrm{~nm}, 10 \mathrm{~mW}, 20 \mathrm{~Hz}, 10 \sim 20 \mathrm{~s}$ ) and mouse behaviors to different experimental targets were examined.

\section{Measurement of behaviors to live predator}

The mice were habituated to the enclosed arena $(25 \mathrm{~cm} \times 25 \mathrm{~cm})$ for 15 minutes per day in consecutive three days before the behavioral test. On the fourth day, after the mice entered the arena, they were first habituated to the arena for 15 minutes to minimize anxiety and stress. Then a live snake $(20 \mathrm{~g}-30 \mathrm{~g})$ was gently placed inside the chamber. To minimize the possibility of predatory attack from the snake, the snake was anaesthetized with isoflurane if necessary. A light-pulse train lasting 10 20 s $(473 \mathrm{~nm}, 5$ $\mathrm{ms}, 20 \mathrm{~Hz}, 10 \mathrm{~mW}$ ) was delivered to stimulate vGAT+ AHN neurons that expressed ChR2-mCherry. The defensive behaviors (risk assessment, freezing, avoidance, biting-like attack) were recorded by the horizontal camera. A researcher blind to the conditions of the mice analyzed the video by plotting a behavioral ethogram off-line. The total time spent for defensive behaviors (risk assessment, freezing, avoidance, or biting-like attack) was used for the quantitative analyses. In some cases (Figure S9, D and $E$ ), we also measured the dependence of defensive attack on the frequency ( $5 \mathrm{~Hz}$, $10 \mathrm{~Hz}, 20 \mathrm{~Hz})$ and power $(2 \mathrm{~mW}, 5 \mathrm{~mW}, 10 \mathrm{~mW})$ of laser pulses.

\section{Measurement of behaviors to neutral object}

The mice were habituated to the enclosed $(25 \mathrm{~cm} \times 25 \mathrm{~cm})$ for 15 minutes per day in consecutive three days before the behavioral test. On the fourth day, after the mice 
entered the arena, they were first habituated to the arena for 15 minutes to minimize anxiety and stress. Then a wood block $(3 \mathrm{~cm} \times 3 \mathrm{~cm} \times 3 \mathrm{~cm})$ was gently placed inside the chamber. For photostimulation of vGAT+ AHN neurons, a light-pulse train lasting 10 20 $\mathrm{s}(473 \mathrm{~nm}, 5 \mathrm{~ms}, 20 \mathrm{~Hz}, 10 \mathrm{~mW})$ was delivered. Mouse behaviors (sniffing, avoidance, freezing, biting-like attack) was recorded by the horizontal camera. A researcher blind to the conditions of the mice analyzed the video by plotting a behavioral ethogram off-line. The total time spent for these behaviors (sniffing, avoidance, freezing, biting-like attack) was used for the quantitative analyses.

\section{Measurement of behaviors to conspecifics}

The mice were habituated to the arena $(25 \mathrm{~cm} \times 25 \mathrm{~cm})$ for 15 minutes per day in consecutive three days before the behavioral test. On the fourth day, after the mice entered the arena, they were first habituated to the arena for 15 minutes to minimize anxiety and stress. Then a male or female C57BL/6 mouse with similar age to the test mice was gently placed inside the chamber. For photostimulation of vGAT+ AHN neurons, a light-pulse train lasting 10 20 s (473 nm, 5 ms, 20 Hz, 10 mW) was delivered. The social behaviors (social investigation, mounting, social attack) was recorded by the horizontal camera. A researcher blind to the conditions of the mice analyzed the video by plotting a behavioral ethogram off-line. The total time spent for social behaviors (social investigation, avoidance, mounting, social attack) was used for the quantitative analyses.

We also tested how light-stimulation of AHN vGAT+ neurons influence social attack in a paradigm of social aggression. A 1-month-old male C57BL/6 mouse was gently placed inside the homecage of test mouse. For photostimulation of vGAT+ AHN neurons, $a$ light-pulse train lasting 10 20 s (473 nm, $5 \mathrm{~ms}, 20 \mathrm{~Hz}, 10 \mathrm{~mW})$ was delivered. The social behaviors (social investigation, mounting, social attack) was recorded by the vertical camera. A researcher blind to the conditions of the mice analyzed the video by plotting a behavioral ethogram off-line. The total time spent for social behaviors (social investigation, avoidance, mounting, social attack) was used for the quantitative analyses. 


\section{Measurement of behaviors to conspecifics and live snake}

The mice were habituated to the arena $(25 \mathrm{~cm} \times 25 \mathrm{~cm})$ for 15 minutes per day in consecutive three days before the behavioral test. On the fourth day, after the mice entered the arena, they were first habituated to the arena for 15 minutes to minimize anxiety and stress. Then a live snake and a male (or female) C57BL/6 mouse with similar age to the test mice were gently placed inside the chamber together. For photostimulation of vGAT+ AHN neurons, a light-pulse train lasting 10 20 s $(473 \mathrm{~nm}, 5$ $\mathrm{ms}, 20 \mathrm{~Hz}, 10 \mathrm{~mW}$ ) was delivered. The social behaviors (social investigation, mounting, social attack) and anti-predator defensive behaviors (freezing, risk assessment, avoidance, defensive attack) were recorded by the horizontal camera. A researcher blind to the conditions of the mice analyzed the video by plotting a behavioral ethogram off-line. The total time spent for each behavior was used for the quantitative analyses.

\section{Cell-type-specific anterograde tracing of AHN vGAT+ neurons}

For cell-type-specific anterograde tracing of vGAT+ AHN neurons, AAV-DIO-EGFP-Syb2 was stereotaxically injected into the AHN of vGAT-ires-Cre mice $(200 \mathrm{nl})$. The mice were then maintained in a cage individually. Three weeks after viral injection, mice were perfused with saline followed by $4 \%$ paraformaldehyde (PFA) in PBS. After $8 \mathrm{~h}$ of post-fixation in 4\% PFA, coronal brain sections at $40 \mu \mathrm{m}$ in thickness were prepared using a cryostat (Leica CM1900). All coronal sections were collected and stained with primary antibody against EGFP and DAPI. The coronal brain sections were imaged with an Olympus VS120 epifluorescence microscope (10× objective lens).

\section{Cannulae implantation of and drug infusion}

A cannula was stereotaxically implanted above the ventrolateral periaqueductal gray (vIPAG). The inner and outer diameters of the cannula were $150 \mu \mathrm{m}$ and $300 \mu \mathrm{m}$, respectively. The cannula was fixed to the skull using acrylic cement. During drug infusion, the cannula was connected with a catheter filled with the picrotoxin (PTX, 100 $\mu \mathrm{M})$ or saline for injection. The other end of the catheter was connected to a Hamilton 
syringe (Hamilton, Reno, NV, USA) controlled by an infusion pump to drive the delivery of PTX or saline ( $50 \mathrm{nl} / \mathrm{min}$ ). The delivery of PTX or saline was operated in a step-wise fashion, with $100 \mathrm{nl}$ infused to the VIPAG in each step $(0,100,200 \mathrm{nl})$. Photostimulation-induced anti-predator defensive behaviors were measured after each step of infusion was completed $(0,100,200 \mathrm{nl})$. At the end of the experimental session, the test mice were perfused and the coronal brain sections containing VIPAG were inspected for the presence of cannula track. The mice with no cannula track above the VIPAG were rejected from further analysis.

\section{Slice physiological recording}

Slice physiological recording was performed according to the published work ${ }^{55}$. Brain slices containing the AHN or PAG were prepared from adult mice anesthetized with isoflurane before decapitation. Brains were rapidly removed and placed in ice-cold oxygenated $\left(95 \% \mathrm{O}_{2}\right.$ and $5 \% \mathrm{CO}_{2}$ ) cutting solution ( $228 \mathrm{mM}$ sucrose, $11 \mathrm{mM}$ glucose, 26 $\mathrm{mM} \mathrm{NaHCO}_{3}, 1 \mathrm{mM} \mathrm{NaH}_{2} \mathrm{PO}_{4}, 2.5 \mathrm{mM} \mathrm{KCl}, 7 \mathrm{mM} \mathrm{MgSO}_{4}$, and $0.5 \mathrm{mM} \mathrm{CaCl}_{2}$ ). Coronal brain slices $(400 \mu \mathrm{m})$ were cut using a vibratome (VT 1200S, Leica Microsystems, Wetzlar, Germany). The slices were incubated at $28^{\circ} \mathrm{C}$ in oxygenated artificial cerebrospinal fluid (ACSF: $119 \mathrm{mM} \mathrm{NaCl}, 2.5 \mathrm{mM} \mathrm{KCl}, 1 \mathrm{mM} \mathrm{NaH}_{2} \mathrm{PO}_{4}, 1.3 \mathrm{mM} \mathrm{MgSO}_{4}$, $26 \mathrm{mM} \mathrm{NaHCO}_{3}, 10 \mathrm{mM}$ glucose, and $2.5 \mathrm{mM} \mathrm{CaCl}_{2}$ ) for $30 \mathrm{~min}$, and were then kept at room temperature under the same conditions for $1 \mathrm{~h}$ before transfer to the recording chamber at room temperature. The ACSF was perfused at $1 \mathrm{ml} / \mathrm{min}$. The acute brain slices were visualized with a 40× Olympus water immersion lens, differential interference contrast (DIC) optics (Olympus Inc., Japan), and a CCD camera.

Patch pipettes were pulled from borosilicate glass capillary tubes (Cat \#64-0793, Warner Instruments, Hamden, CT, USA) using a PC-10 pipette puller (Narishige Inc., Tokyo, Japan). For recording of action potentials (current clamp), pipettes were filled with solution (in mM: 135 K-methanesulfonate, 10 HEPES, 1 EGTA, 1 Na-GTP, 4 Mg-ATP, and $2 \%$ neurobiotin, $\mathrm{pH}$ 7.4). For recording of postsynaptic currents (voltage 
clamp), pipettes were filled with solution (in $\mathrm{mM}, 135 \mathrm{CsCl}, 10$ HEPES, 1 EGTA, 1 Na-GTP, 4 Mg-ATP, pH 7.4). The resistance of pipettes varied between 3.0-3.5 M 2 . The current and voltage signals were recorded with MultiClamp 700B and Clampex 10 data acquisition software (Molecular Devices). After establishment of the whole-cell configuration and equilibration of the intracellular pipette solution with the cytoplasm, series resistance was compensated to 10-15 M 2 . Recordings with series resistances of $>15 \mathrm{M} \Omega$ were rejected.

An optical fiber (230 $\mu \mathrm{m}$ in diameter, N.A. 0.37) was used to deliver light pulses, with the fiber tip positioned $500 \mu \mathrm{m}$ above the brain slices. Laser power was adjusted to 10 $\mathrm{mW}$. Light-mediated photoinhibition of GtACR1+ neurons was tested by a constant laser illumination ( $473 \mathrm{~nm}, 300 \mathrm{~ms}, 10 \mathrm{~mW}$ ) while the neurons were depolarized (usually 0.1 $\mathrm{nA}$ ) to trigger action potential firing (Figure 2B). Light-evoked action potentials from ChR2-mCherry ${ }^{+}$neurons in the AHN were triggered by a light-pulse train $(473 \mathrm{~nm}, 2 \mathrm{~ms}$, $10 \mathrm{~Hz}$ or $20 \mathrm{~Hz}, 10 \mathrm{~mW}$ ) synchronized with Clampex 10 data acquisition software (Molecular Devices) (Figure 4C). Light-evoked postsynaptic currents from PAG neurons were triggered by single light pulses (2 ms) in the presence of 4-aminopyridine (4-AP, 20 $\mu \mathrm{M})$ and tetrodotoxin (TTX, $1 \mu \mathrm{M})$. D-AP5 $(50 \mu \mathrm{M}) / \mathrm{CNQX}(20 \mu \mathrm{M})$ or picrotoxin (PTX, 50 $\mu \mathrm{M})$ were perfused with ACSF to examine the neurotransmitter type used by ChR2-mCherry-expressing AHN neurons (Figure 5, E and F).

\section{RNA in situ hybridization}

Mice were perfused with PBS treated with 0.1\% DEPC (Sigma, D5758), followed by DEPC-treated PBS containing 4\% PFA (PBS-PFA). Brains were post-fixed in DEPC-treated PBS-PFA solution overnight and then placed in DEPC-treated $30 \%$ sucrose solution at $4^{\circ} \mathrm{C}$ for $30 \mathrm{~h}$. Brain sections to a thickness of $30 \mu \mathrm{m}$ were prepared using a cryostat (Leica, CM3050S) and collected in DPEC-treated PBS. Fluorescence in situ hybridization (FISH) was performed as previously described (Chen et al., 2020) with minor modifications. Briefly, brain sections were rinsed with DPEC-treated PBS, 
permeabilized with DPEC-treated $0.1 \%$ Tween 20 solution (in PBS) and DPEC-treated 2 $\times$ SSC containing $0.5 \%$ Triton. Brain sections were then treated with $\mathrm{H} 2 \mathrm{O} 2$ solution and acetic anhydride solution to reduce nonspecific FISH signals. After $2 \mathrm{~h}$ incubation in prehybridization buffer ( $50 \%$ formamide, $5 \times$ SSC, $0.1 \%$ Tween $20,0.1 \%$ CHAPS, $5 \mathrm{mM}$ EDTA in DEPC-treated water) at $65^{\circ} \mathrm{C}$, brain sections were then hybridized with the hybridization solution containing mouse antisense cRNA probes (digoxigenin labeling) for vGlut2 (amplified by primers CCAAATCTTACGGTGCTACCTC and TAGCCATCTTTCCTGTTCCACT) or VGAT (amplified by primers GCCATTCAGGGCATGTTC and AGCAGCGTGAAGACCACC) at $65^{\circ} \mathrm{C}$ for 20h. The sequences of cDNA primers for cRNA probes were the same as those in the ISH DATA of the Allen brain atlas (https://mouse.brain-map.org/). After washing, brain sections were incubated with Anti-Digoxigenin-POD, Fab fragments (1:400, Roche, 11207733910) at $4^{\circ} \mathrm{C}$ for $30 \mathrm{~h}$, and FISH signals were detected using a TSA Plus Cyanine 3 kit (NEL744001KT, PerkinElmer). To detect the GFP signals, brain sections were incubated with a primary antibody against GFP(1:2000, Abcam ab290) at $4^{\circ} \mathrm{C}$ for $24 \mathrm{~h}$ and then with an Alexa Fluor ${ }^{\circledR}$ 488-conjugated goat anti-rabbit secondary antibody (1:500, Invitrogen, A11034) at room temperature for $2 \mathrm{~h}$. Brain sections were mounted and imaged using a Zeiss LSM780 confocal microscope or the Olympus VS120 Slide Scanning System.

\section{Immunohistochemistry}

Mice were anesthetized with isoflurane and sequentially perfused with saline and phosphate buffered saline (PBS) containing 4\% paraformaldehyde (PFA). Brains were removed and incubated in PBS containing 30\% sucrose until they sank to the bottom. Post-fixation of the brain was avoided to optimize immunohistochemistry of GABA and glutamate. Cryostat sections $(40 \mu \mathrm{m})$ were collected, incubated overnight with blocking solution (PBS containing 10\% goat serum and $0.7 \%$ Triton X-100), and then treated with primary antibodies diluted with blocking solution for 3-4 h at room temperature. Primary antibodies used for immunohistochemistry are displayed in Table S1. Primary antibodies 
were washed three times with washing buffer (PBS containing $0.7 \%$ Triton X-100) before incubation with secondary antibodies (tagged with Cy2, Сy3, or Cy5; dilution 1:500; Life Technologies Inc., USA) for $1 \mathrm{~h}$ at room temperature. Sections were then washed three times with washing buffer, stained with DAPI, and washed with PBS, transferred onto Super Frost slides, and mounted under glass coverslips with mounting media.

Sections were imaged with an Olympus VS120 epifluorescence microscope (10x objective lens) or a Zeiss LSM 710 confocal microscope (20x and 60x oil-immersion objective lens). Samples were excited by 488,543 , or $633 \mathrm{~nm}$ lasers in sequential acquisition mode to avoid signal leakage. Saturation was avoided by monitoring pixel intensity with Hi-Lo mode. Confocal images were analyzed with ImageJ software.

\section{Analyses of cell-type specificity}

Two types of experiments were performed to quantify cell-type specificity in the study. First, we tested the specificity of vGAT-IRES-Cre and vGlut2-IRES-Cre lines to label vGAT+ and vGlut2+ AHN neurons, by injecting AAV-DIO-EGFP into the AHN of these mice. Then we collected the tissue sections of $\mathrm{AHN}$ and analyzed the expression of vGAT/vGlut2 mRNA and EGFP were examined by RNA in situ hybridization and immunohistochemistry, respectively. The specificity and efficiency of vGAT-IRES-Cre and vGlut2-IRES-Cre mice to label vGAT+ and vGlut2+ neurons were calculated (Figure S4D-S4O).

Second, we tested whether the molecular tools (GtACR1-2A-EGFP, jGCaMP7s, ChR2-mCherry) were specifically expressed in GABAergic neurons in the AHN, by immunostaining of GABA with an anti-GABA antibody that has been validated in our previous studies (Shang et al., 2018). The cell-type specificity and efficiency for each molecular tool to express in VGAT+ AHN neurons was quantitatively analyzed (GtACR1-2A-EGFP: Figure S5C-S5F; jGCaMP7s: Figure S6A and S6B; ChR2-mCherry: Figure 4B and S9A).

\section{Data quantification and statistical analyses}


1134 All experiments were performed with anonymized samples in which the experimenter 1135 was unaware of the experimental conditions of the mice. For the statistical analyses of 1136 experimental data, Student t-test and One-Way ANOVA were used. The " $\mathrm{n}$ " used for 1137 these analyses represents number of mice or cells. See the detailed information of 1138 statistical analyses in figure legend and in Table S4. All statistical comparisons were conducted on data originating from three or more biologically independent experimental replicates. All data are shown as means \pm SEM.

Data availability

1142 The data that support the findings of this study are available from the corresponding 1143 author upon reasonable request. 
Supplementary Materials

\section{Hypothalamic circuits for mechanically-evoked defensive attack}

Zhiyong Xie, Huating Gu, Congping Shang, Xinyu Cheng, Meizhu Huang, Dapeng Li, Ting Tao, Yuan Xie, Jidong Zhao, Wei Lu, Zhibing Zhang, Zongxiang Tang, Cheng Zhan, Fan Zhang, Peng Cao

This file includes:

Methods

Figure S1 to Figure S16

Movies S1 to S15

Tables S1 to S4 

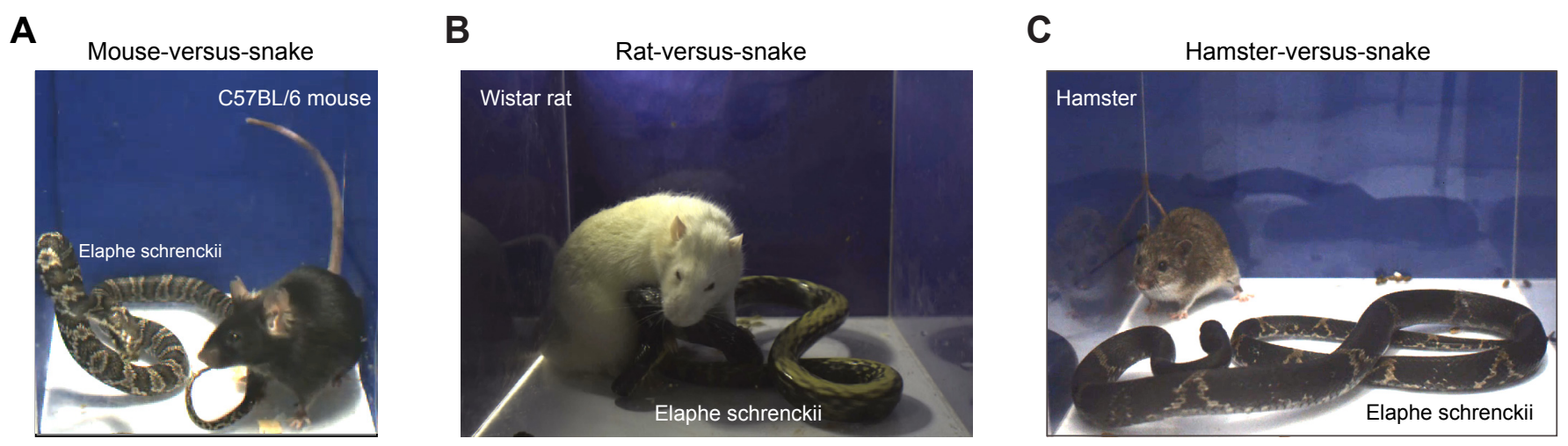

Figure S1 Xie et al., 2020 
$1 \quad$ Figure S1 Rodent-versus-Snake paradigms. (Related to Figure 1)

2 (A) An example picture showing the mouse-versus-snake paradigm. In an enclosed 3 arena $(10 \mathrm{~cm} \times 10 \mathrm{~cm})$, one adult male C57BL/6 mouse was exposed to a one-month old 4 snake (Elaphe schrenckii) with similar weight to the mouse. See Movie S1 for more 5 details.

6 (B) An example picture showing the rat-versus-snake paradigm. In an enclosed arena 7 (25 cm $\times 25 \mathrm{~cm})$, one adult male Wistar rat was exposed to a three-month old snake 8 (Elaphe schrenckii) with similar weight to the rat. See Movie S2 for more details.

9 (C) An example picture showing the hamster-versus-snake paradigm. In an enclosed 10 arena $(25 \mathrm{~cm} \times 25 \mathrm{~cm})$, one adult male Greater long-tailed hamster (Tscherskia triton) 11 was exposed to a three-month old snake (Elaphe schrenckii) with similar weight to the hamster. See Movie S3 for more details. 

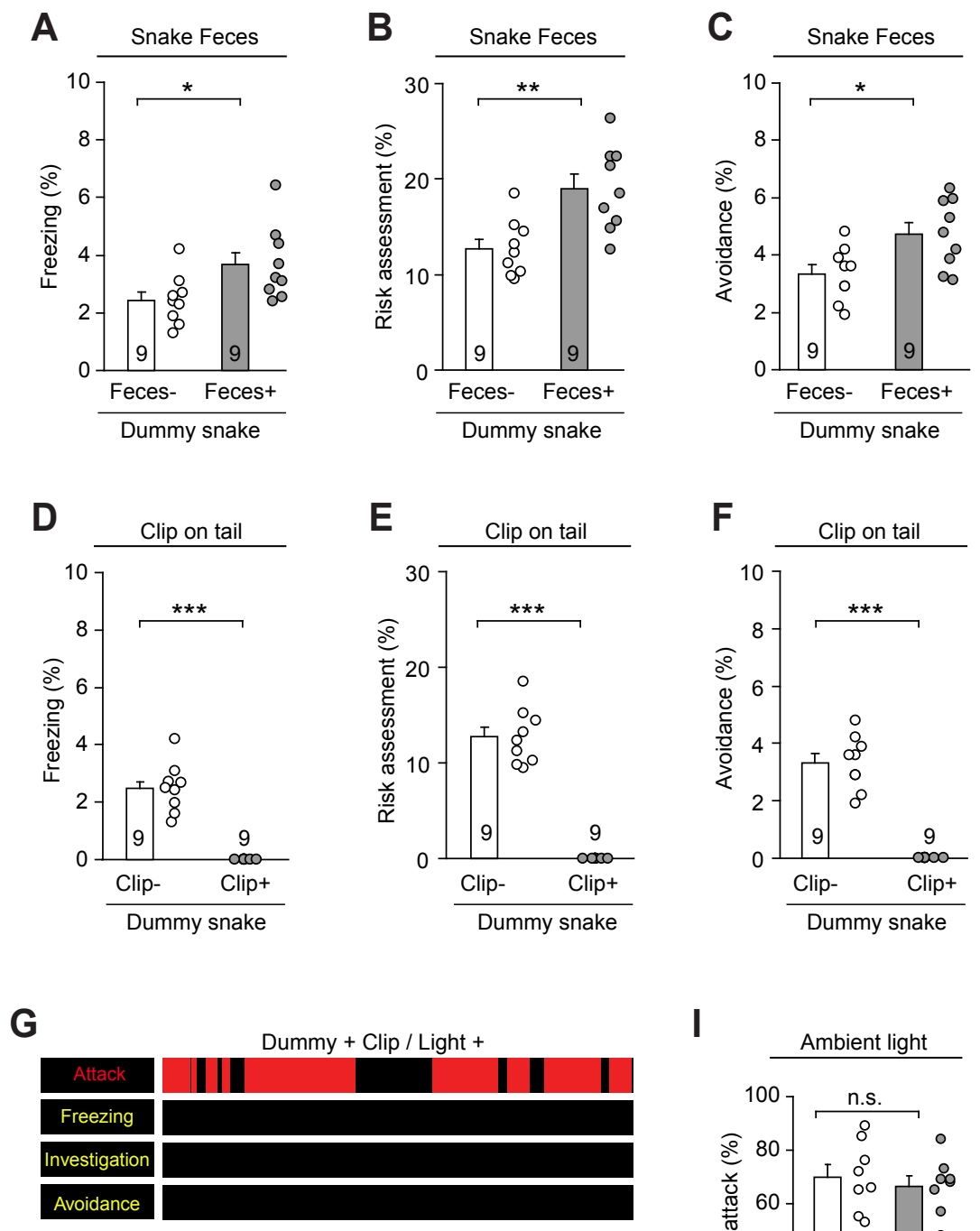

H
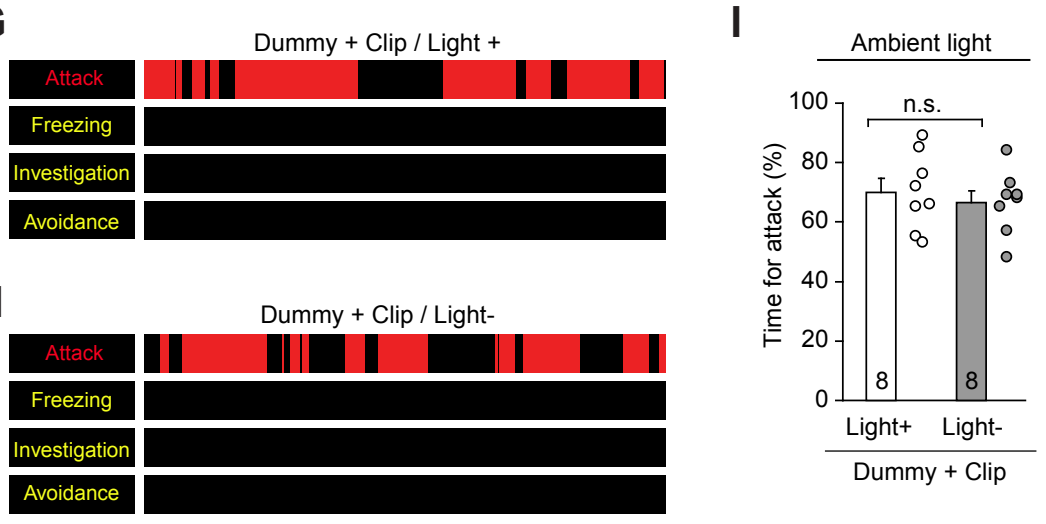

\section{J}

Clip on limbs

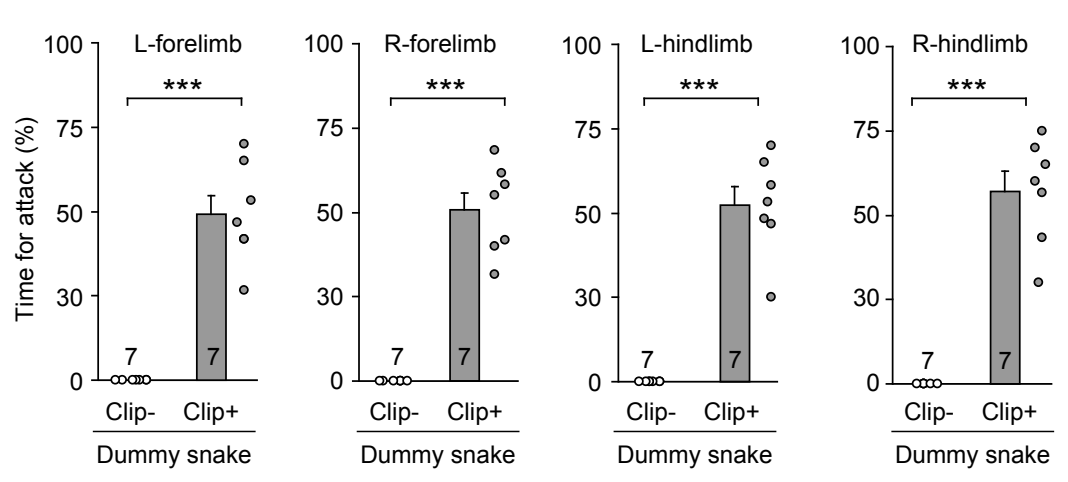

Figure S2 Xie et al., 2020 
Figure S2 More analyses of sensory-triggered defensive behaviors. (Related to Figure 1)

(A-C) Quantitative analyses of time spent on freezing (A), risk assessment (B), and avoidance $(C)$ in response to a dummy snake coated with or without snake feces.

(D-F) Quantitative analyses of time spent on freezing (D), risk assessment (E), and avoidance $(F)$ of mice in response to a dummy snake with or without an alligator-clip to provide noxious mechanical stimuli.

(G-I) Example behavioral ethograms $(\mathrm{G}, \mathrm{H})$ and quantitative analyses of time spent for biting attack (I) of mice in response to a dummy snake providing noxious mechanical stimuli on the tail in an enclosed arena with or without ambient light. The colored bars in the ethograms indicated the onset and offset of specific behaviors. The colored bars in the ethograms indicated the onset and offset of specific behaviors.

(J) Quantitative analyses of time spent for biting attack of mice in response to a dummy snake with or without providing mechanical stimuli on four limbs.

Numbers of mice $(A-F, I, J)$ are indicated in the graphs. Data in $(A-F, I, J)$ are means \pm SEM. Statistical analyses in (A-F, I, J) were performed by Student t-tests (n.s. P>0.1, * $\left.\mathrm{P}<0.05 ;{ }^{* *} \mathrm{P}<0.01 ;{ }^{* *} \mathrm{P}<0.001\right)$. For the $\mathrm{P}$ values, see the Table S4. 
A

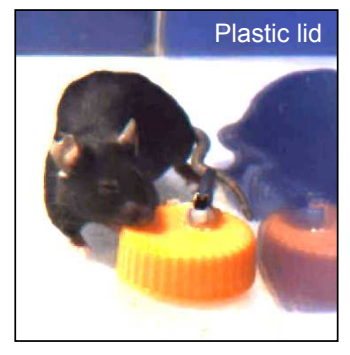

B

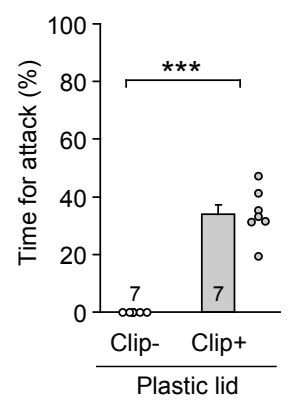

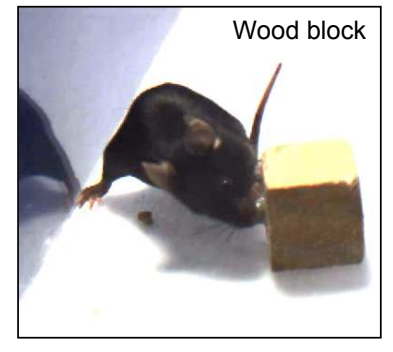

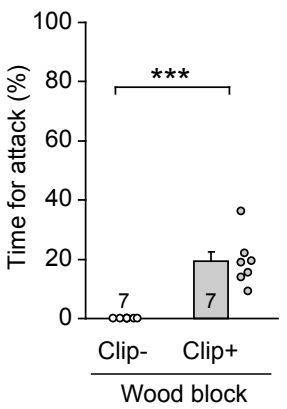

Figure S3 Xie et al., 2020 
Figure S3 Neutral object linked to noxious mechanical stimuli evoked defensive attack. (Related to Figure 1)

(A, B) Example picture (left) and quantitative analyses of time for attack (right) showing biting-like attack toward a plastic lid (A) and a wood block (B) that were linked to noxious mechanical stimuli in an enclosed arena. For more details, see Movie S6.

Numbers of mice are indicated in the graphs (A, B). Data are means \pm SEM. Statistical analyses in $(A, B)$ were performed by Student $t$-tests $\left({ }^{* * *} P<0.001\right)$. For the $P$ values,

37 see the Table S4. 
A

vGat mRNA
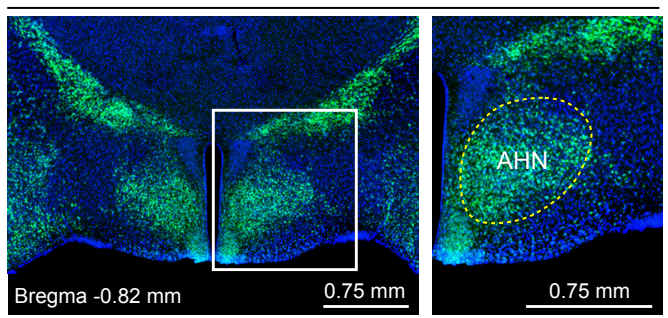

E

$v$ GAT-IRES-Cre mice / AAV-DIO-EGFP

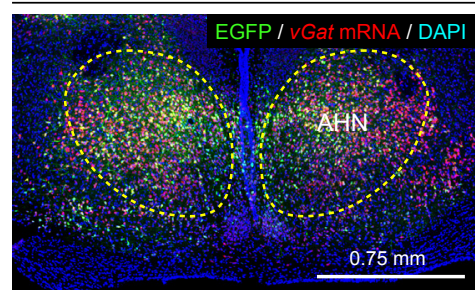

G

VGAT-IRES-Cre mice / AAV-DIO-EGFP

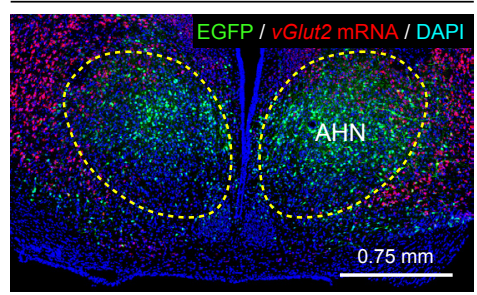

J

vGlut2-IRES-Cre mice / AAV-DIO-EGFP

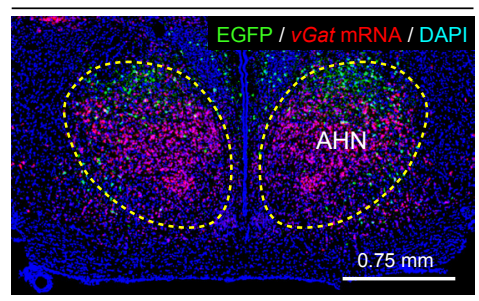

M

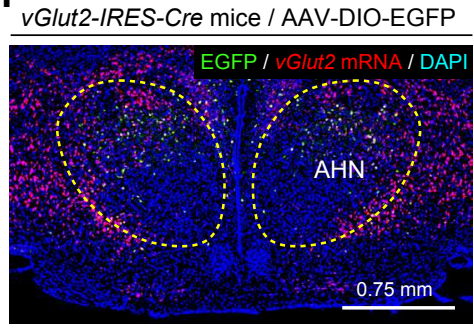

H

K

N
B

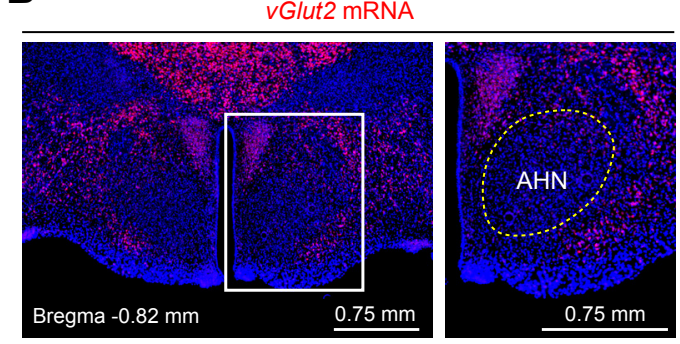

C

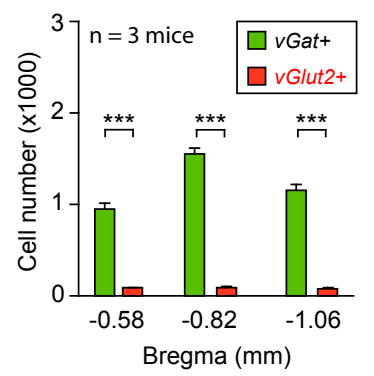

F
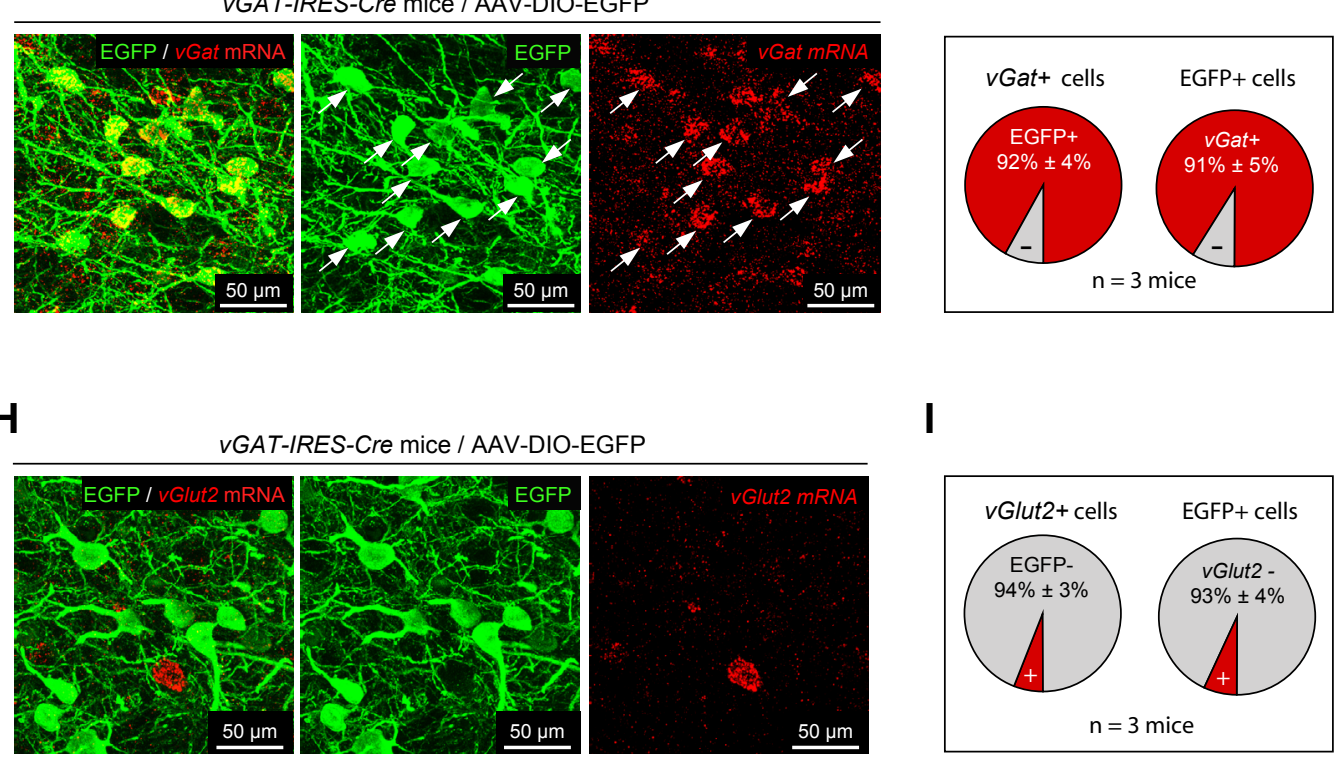

I

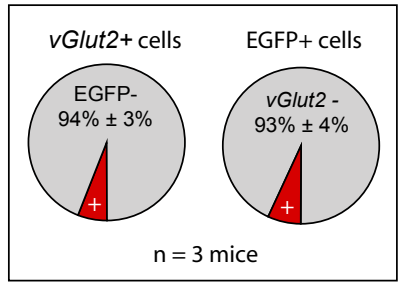

vGlut2-IRES-Cre mice / AAV-DIO-EGFP
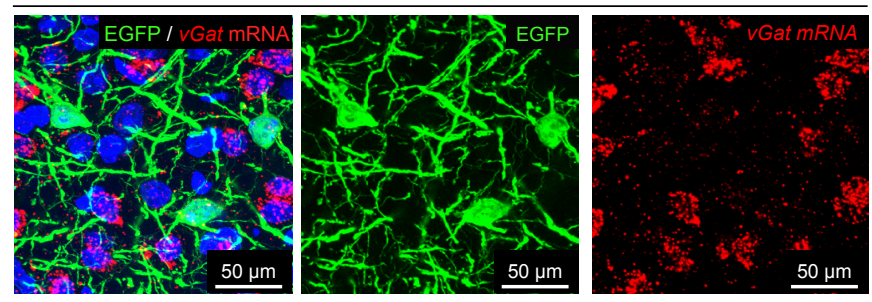

$\mathbf{L}$

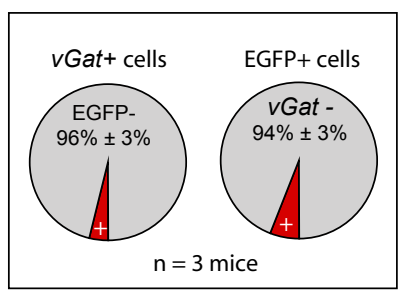

vG/ut2-IRES-Cre mice / AAV-DIO-EGFP
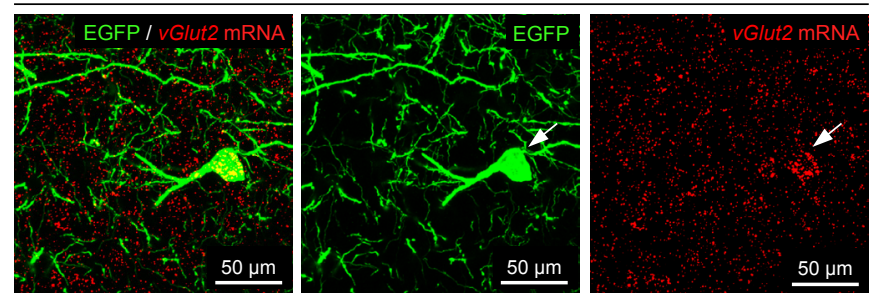

0

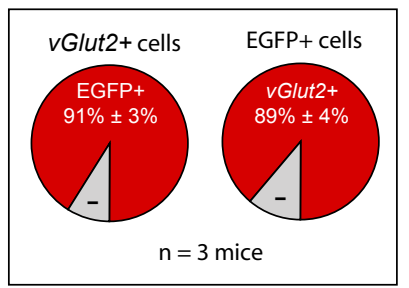

Figure S4 Xie et al., 2020 
Figure S4 Analyses of cell-type specificity of vGAT-IRES-Cre and vGlut2-IRES-Cre lines in the AHN. (Related to Figure 2)

(A, B) Example micrographs of fluorescent in situ hybridization showing vGat mRNA (A) and vGlut2 mRNA (B) distributed in the AHN of WT mice.

(C) Quantitative analyses of number of AHN cells expressing vGat mRNA (vGat+) and vglut2 mRNA (vGlut2+) in coronal sections, as indicated by the distance to bregma.

(D-F) An example coronal section showing the distribution of EGFP (green) and $v G a t$ mRNA (red) in the AHN of vGAT-IRES-Cre mice, which were injected with AAV-DIO-EGFP in the AHN (D). Example micrographs (E) and statistical analyses (F) showing EGFP and $v$ Gat mRNA were mostly colocalized in the same AHN neurons.

(G-I) An example coronal section showing the distribution of EGFP (green) and vGlut2 mRNA (red) in the AHN of vGAT-IRES-Cre mice, which were injected with AAV-DIO-EGFP in the AHN (G). Example micrographs $(H)$ and statistical analyses (I) showing EGFP and vGlut2 mRNA were largely segregated in different AHN neurons.

(J-L) An example coronal section showing the distribution of EGFP (green) and vGat mRNA (red) in the AHN of vGlut2-IRES-Cre mice, which were injected with AAV-DIO-EGFP in the AHN (J). Example micrographs $(\mathrm{K})$ and statistical analyses $(\mathrm{L})$ showing EGFP and $v G a t$ mRNA were mostly segregated from different AHN neurons.

(M-O) An example coronal section showing the distribution of EGFP (green) and vGlut2 mRNA (red) in the AHN of vGlut2-IRES-Cre mice, which were injected with AAV-DIO-EGFP in the AHN (M). Example micrographs ( $N$ ) and statistical analyses (O) showing EGFP and vGlut2 mRNA were mostly colocalized in the same AHN neurons.

Arrows indicate the dually-labeled cells $(E, N)$. Scale bars are indicated in the graphs. Numbers of mice are indicated in the graphs (C, F, I, L, O). Data in (C, F, I, L, O) are means \pm SEM. 
A

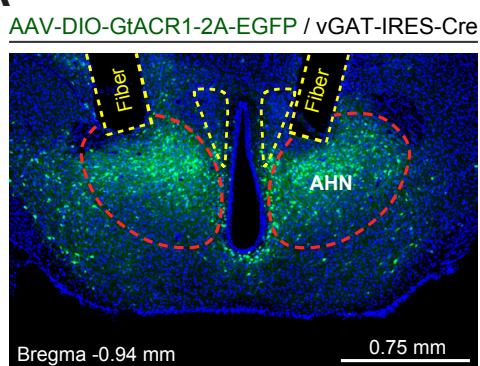

B

AAV-DIO-GtACR1-2A-EGFP / vGlut2-IRES-Cre

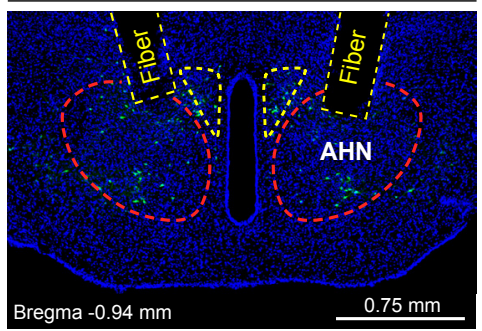

E

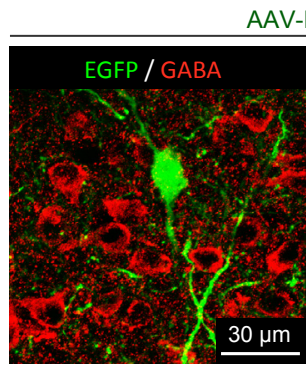

AAV-DIO-GtACR1-2A-EGFP / vGlut2-IRES-Cre

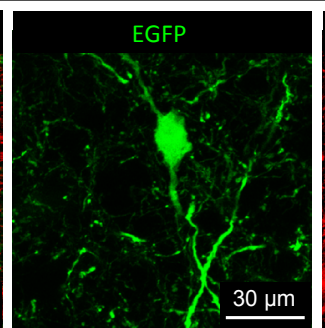

G

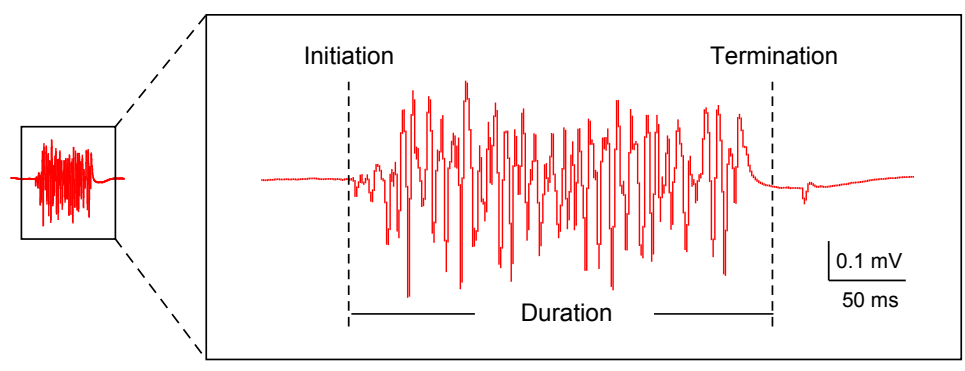

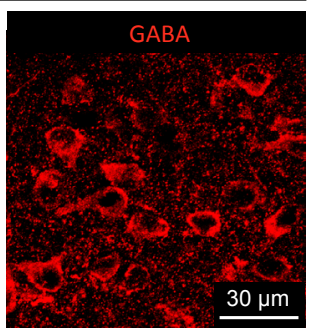

F

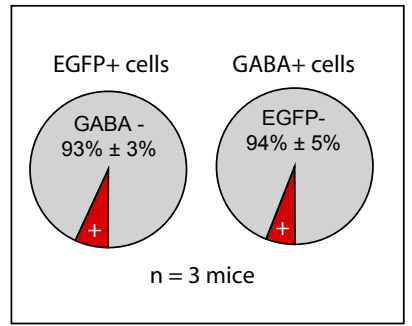

D

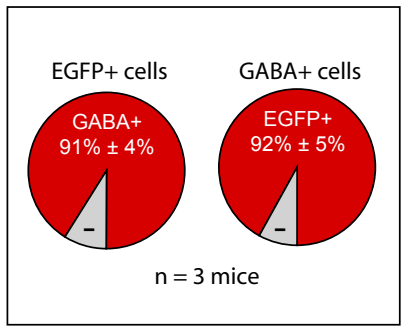

H

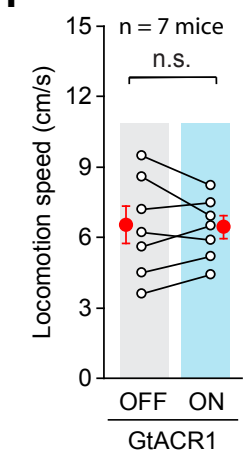

Figure S5 Xie et al., 2020 
Figure S5 AHN vGAT+ neurons are selectively required for mechanically-evoked defensive attack. (Related to Figure 2)

(A) An example coronal section showing EGFP expression in the AHN of vGAT-IRES-Cre mice, and bilateral optical fiber tracks above the AHN.

(B) An example coronal section showing EGFP expression in the AHN of vGlut2-IRES-Cre mice, and bilateral optical fiber tracks above the AHN. Note the EGFP+ cells are sparsely distributed in the AHN.

(C, D) Example micrographs (C) and quantitative analyses (D) showing the EGFP+ AHN neurons in the vGAT-IRES-Cre mice are mostly GABA-positive.

(E, F) Example micrographs $(E)$ and quantitative analyses $(F)$ showing the EGFP+ AHN neurons in the vGlut2-IRES-Cre mice are mostly GABA-negative.

(G) An example EMG trace recorded from the masseter muscles showing the initiation and termination of biting attack, as indicated by the two dashed vertical lines.

(H) Quantitative analyses of locomotion speed before (OFF) and during (ON) photoinhibition of AHN vGAT+ neurons of freely moving mice.

Scale bars are labeled in the graphs. Data in (D, F, H) are means \pm SEM (error bars). Numbers of mice are indicated in the graphs (D, F, H). Statistical analyses were performed by Student $t$-tests (n.s., $\mathrm{P}>0.1$ ). For the $\mathrm{P}$ values, see Table $\mathrm{S} 4$. 
A
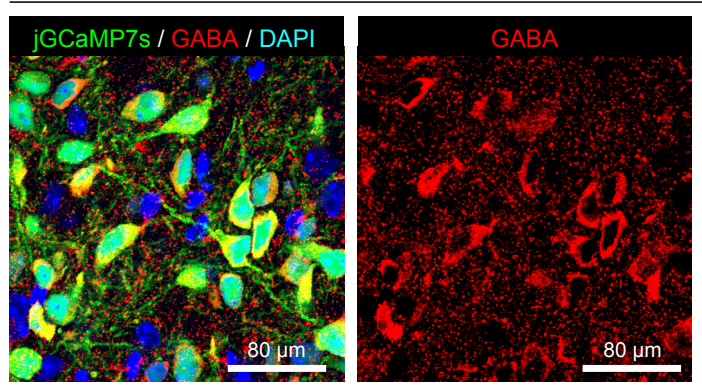

B

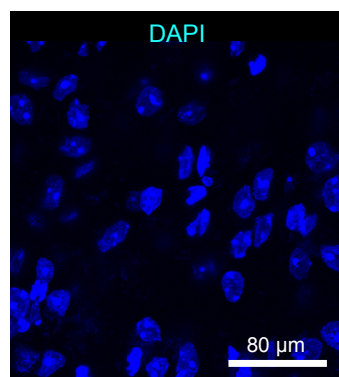

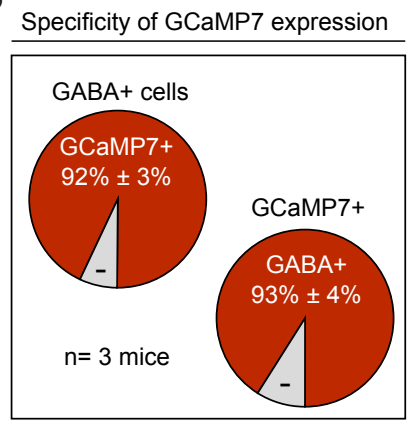

C
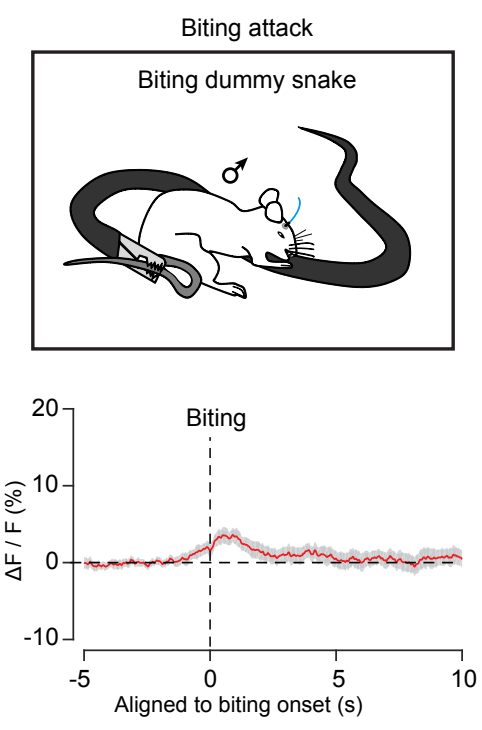

F

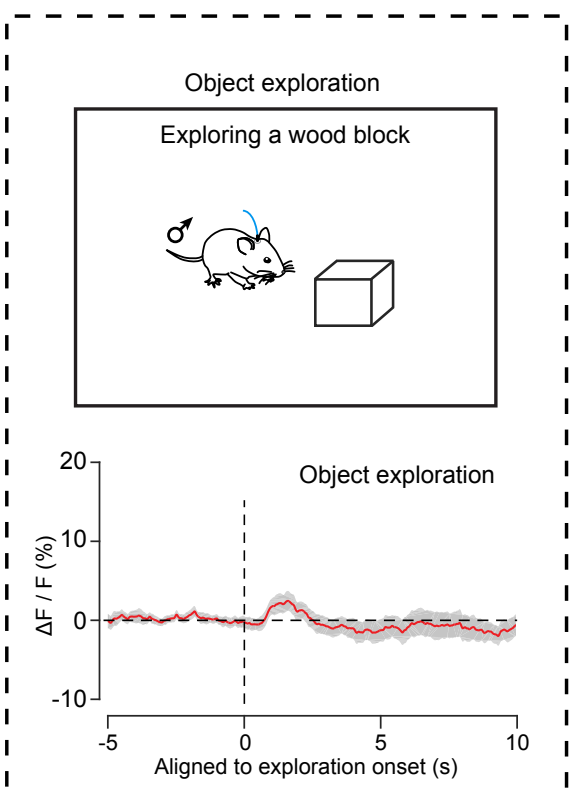

D

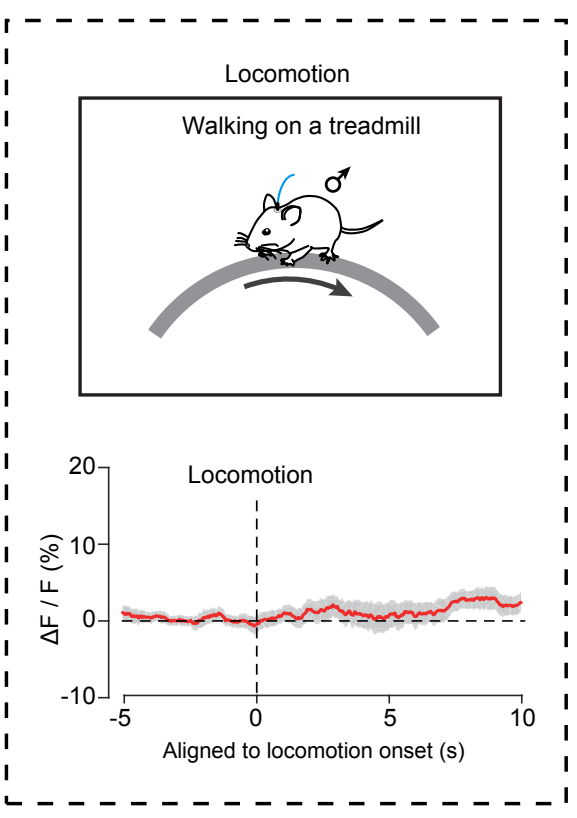

G

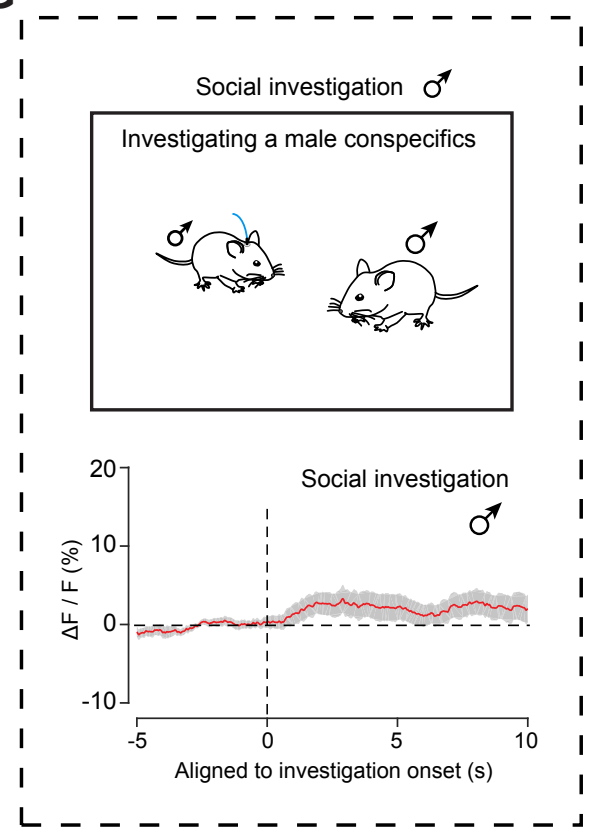

E
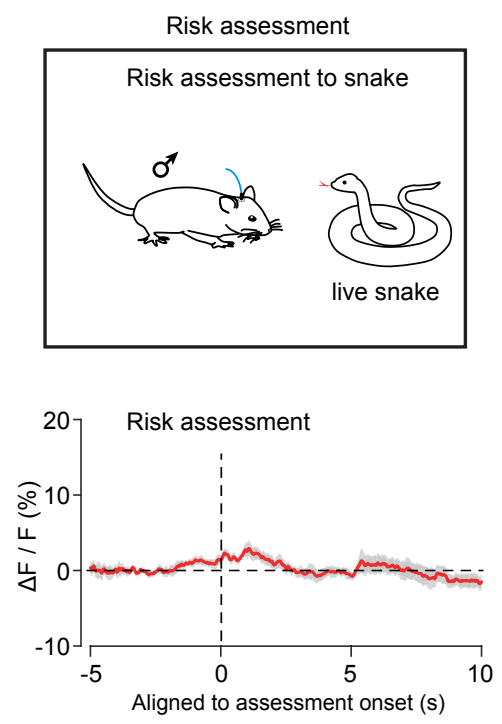

H

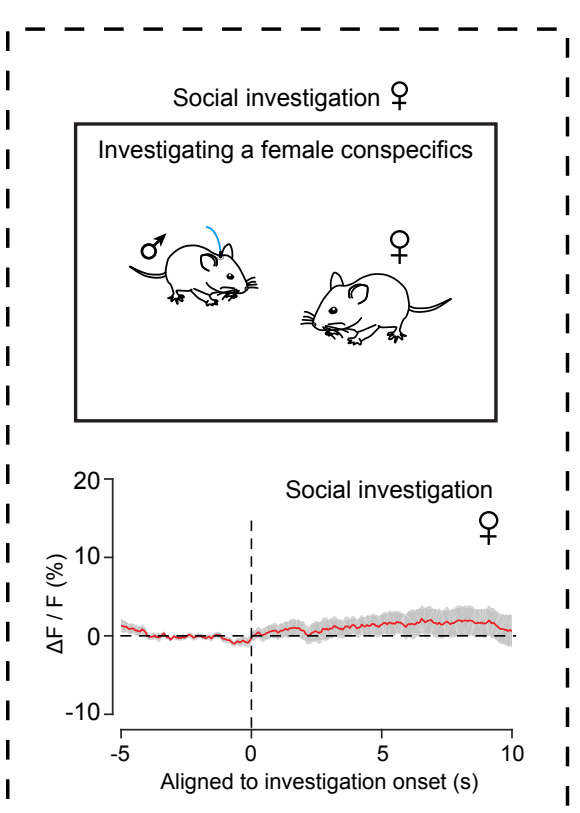


Figure S6 Fiber photometry recording of GCaMP signals from AHN VGAT+ neurons. (Related to Figure 3)

(A, B) Example micrographs with immunostaining of EGFP and GABA $(A)$ and quantitative analyses (B) showing specific expression of jGCaMP7s in GABA+ AHN neurons in vGAT-IRES-Cre mice.

(C-H) Schematic diagrams (top) and normalized GCaMP-fluorescence traces of example mice (bottom) showing the activity of AHN vGAT+ neurons in different behavioral tests, including biting dummy snake evoked by mechanical stimuli $(C)$, walking on a treadmill $(D)$, risk assessment to snake $(E)$, exploring a wood block $(F)$, investigating a male conspecifics $(G)$ and a female conspecifics $(H)$. Dashed lines indicate the onset of the behaviors based on visual inspection of the videos taken with high-speed camera. Scale bars are labeled in the graphs $(A)$. Data in $(B-H)$ are means \pm SEM (error bars). Number of mice is indicated in the graphs (B). 
A

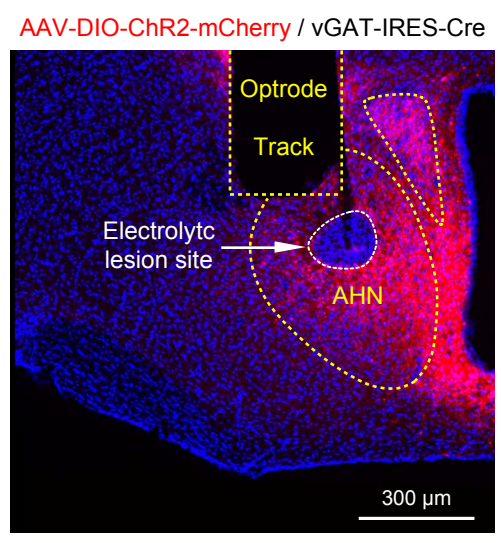

C

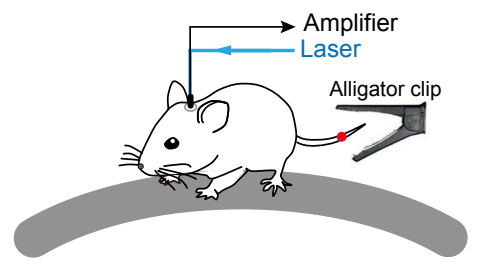

D

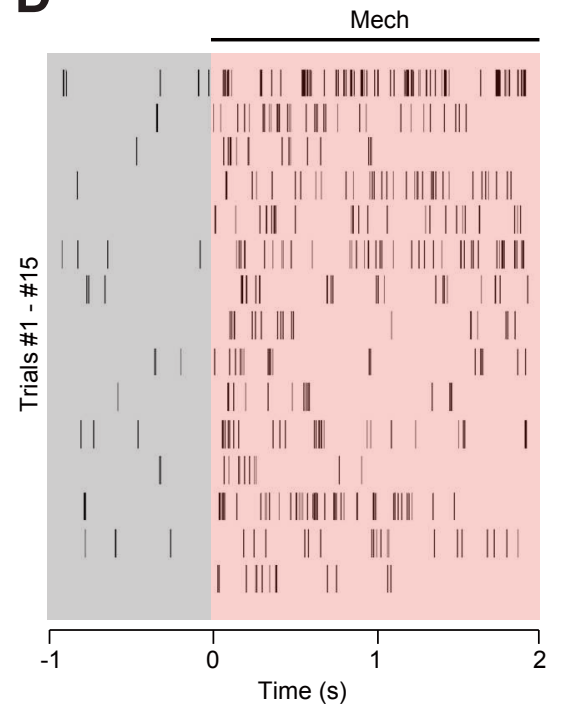

I

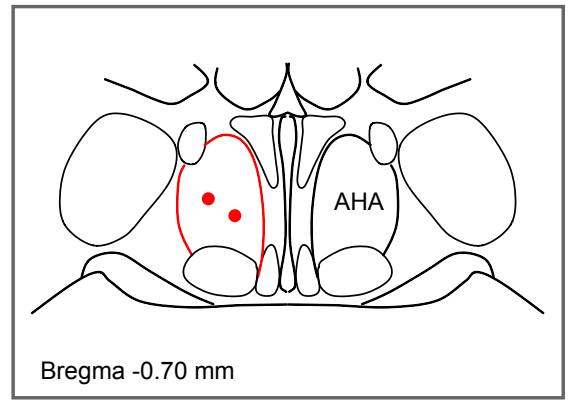

B
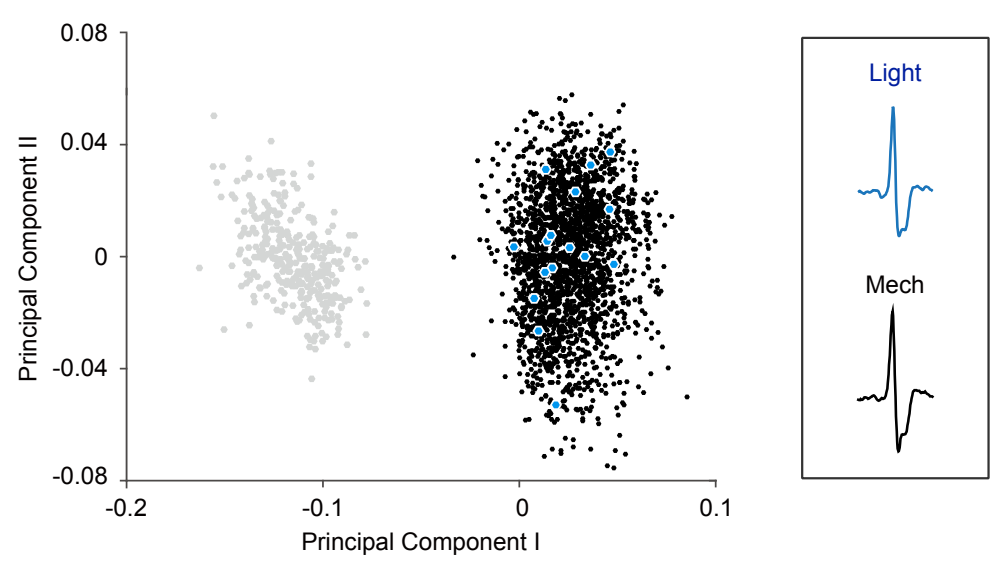

E

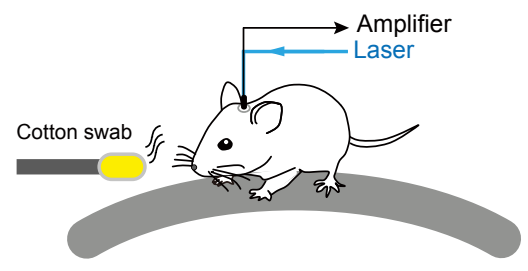

G

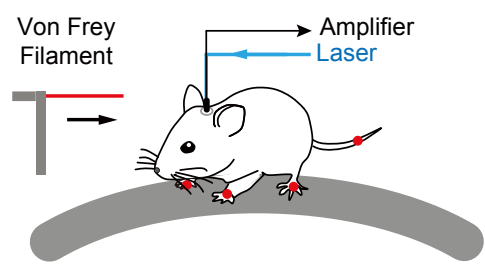

F

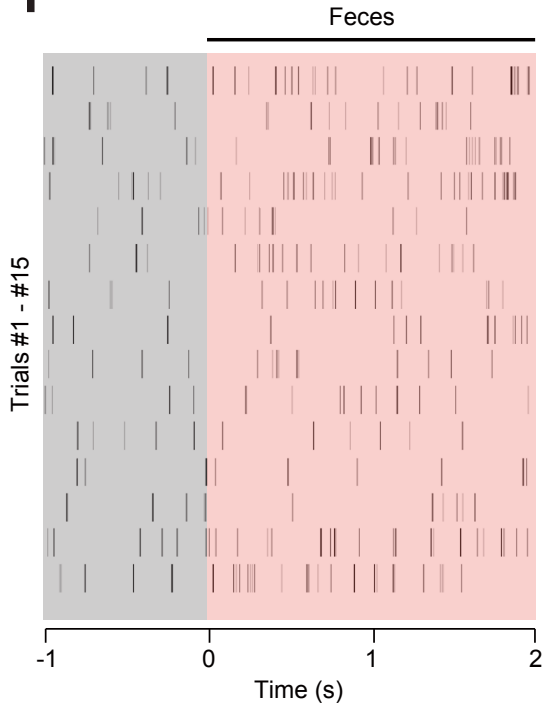

H

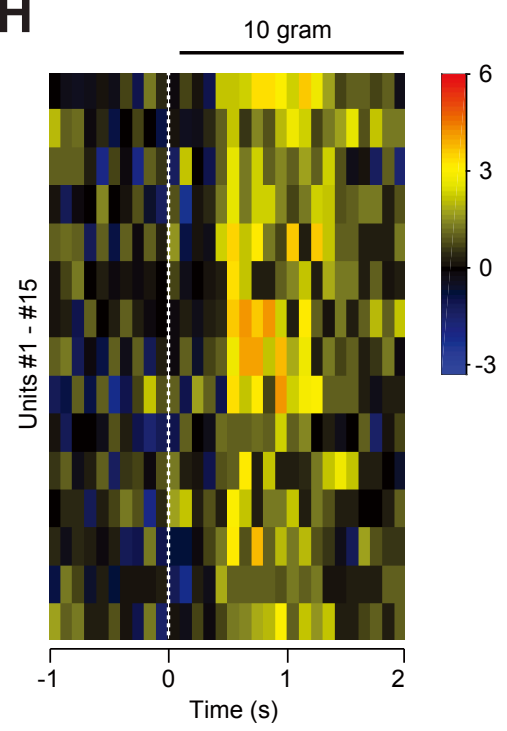

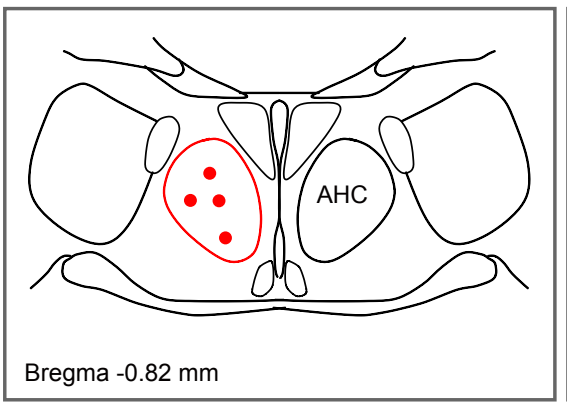

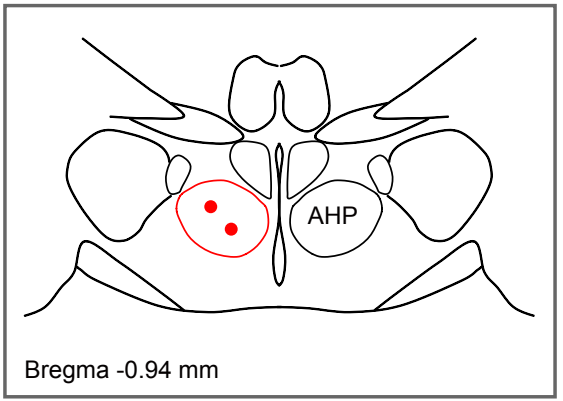

Figure S7 Xie et al., 2020 
Figure S7 Single-unit recording from AHN vGAT+ neurons with an optrode. (Related to Figure 3)

(A) An example coronal section showing ChR2-mCherry expression in the AHN of vGAT-IRES-Cre mice, and an optrode track above the AHN. Note the recording site was marked by electrolytic lesion (arrow) in the AHN. For the analyses of specific expression of ChR2-mCherry in GABA+ AHN neurons, see Figure 4B and Figure S9A.

(B) Principal component analysis of light-evoked spikes (blue dots) and mechanically-evoked spikes (black dots) of an example putative AHN vGAT+ neuron. Gray dots represent noise. Inset shows example waveforms of light-evoked spike (Light) and mechanically-evoked spike (Mech).

(C, E) Schematic diagrams showing the application of mechanical stimuli on the tail with an alligator-clip (C) and the application of olfactory stimuli by presenting cotton swab with snake feces $(E)$ to the test mice.

(D, F) Raster plots of example units of putative vGAT+ AHN neurons showing their responses to mechanical stimuli (Mech) (D) and to cotton swab with snake feces (Feces) $(\mathrm{F})$.

(G) A schematic diagram showing the application of von Frey Filaments poking different body parts of the test mice (tail, four limbs). Red dots indicated the site for poking.

(H) Heat-map PSTH of Z-scored firing rates of individual vGAT+ AHN neurons to mechanical stimuli applied with von Frey Filaments $(10 \mathrm{~g})$.

(I) Schematic diagrams showing the recording sites marked by electrolytic lesions were within the anterior part (AHA), central part (AHC) and posterior part (AHP) of the AHN. 
A
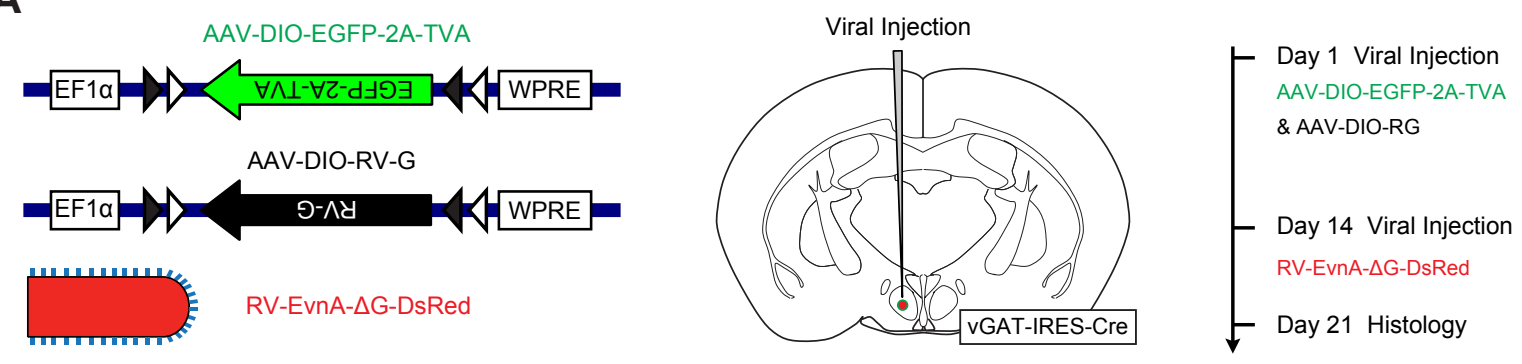

B
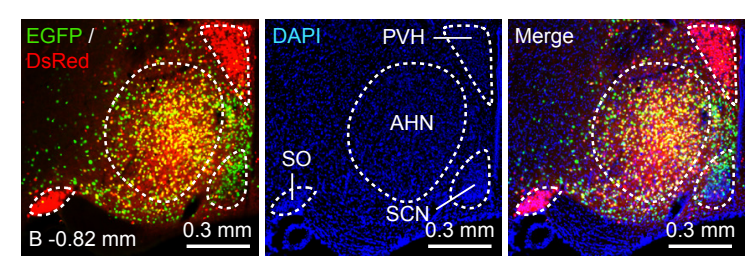

C
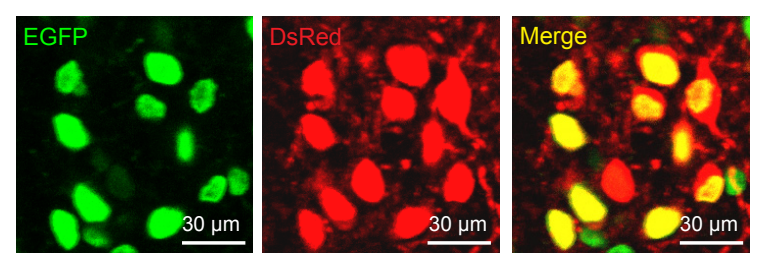

D
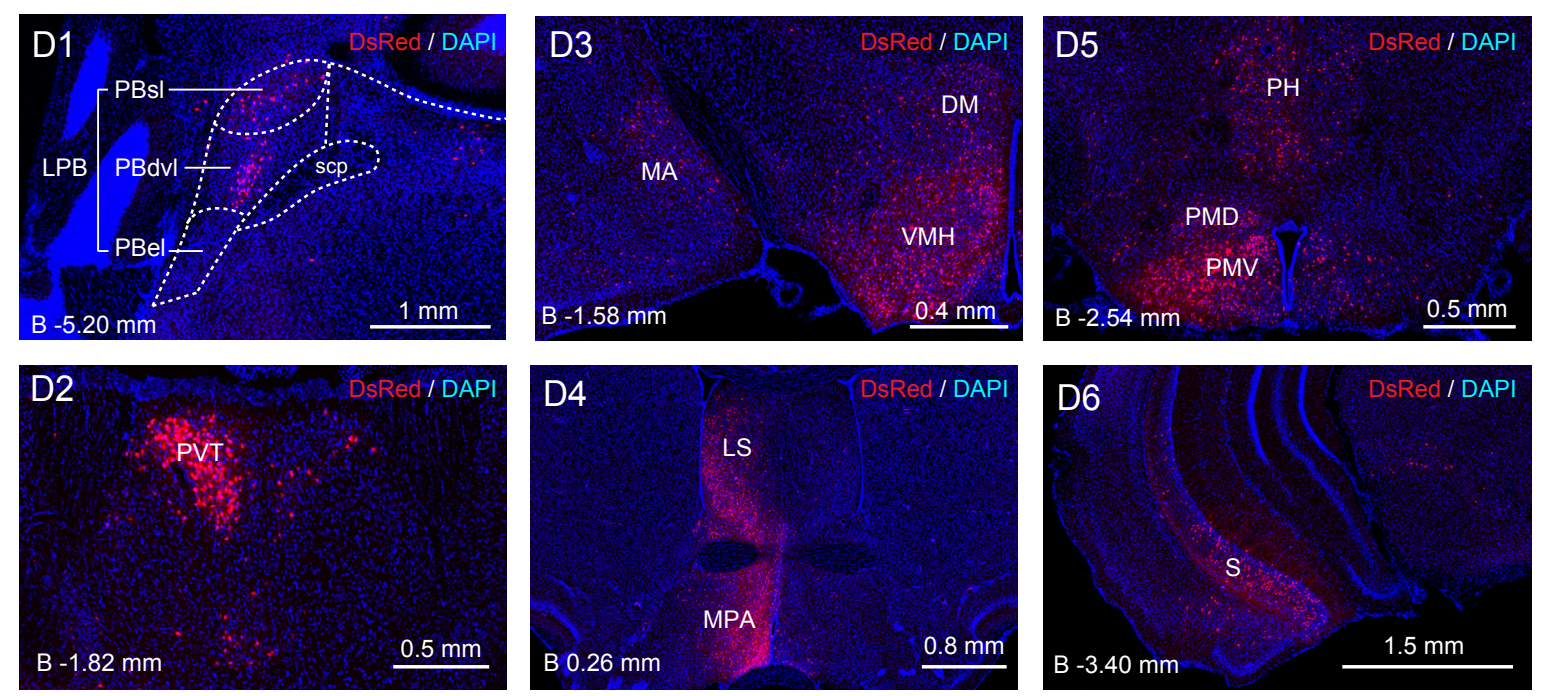

E

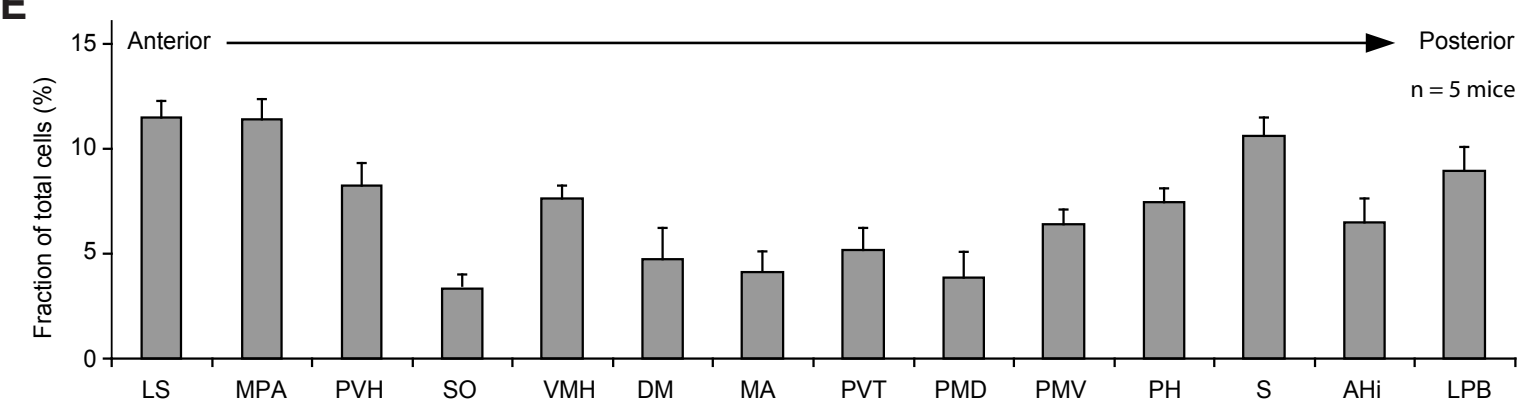

Figure S8 Xie et al., 2020 
Figure S8 Retrograde tracing of AHN vGAT+ neurons by rabies virus. (Related to

\section{Figure 3)}

(A) A series of schematic diagrams showing the strategy for monosynaptic retrograde tracing of AHN vGAT+ neurons by using a combination of AAV and rabies virus (RV). Left, a diagram showing $\mathrm{AAV}$ and $\mathrm{RV}$ for brain injection. Middle, a diagram showing the brain area for injection. Right, a diagram showing the timing of $A A V$ injection and RV injection.

(B) Example micrographs showing that the viral injection center, as indicated by the co-expression of EGFP and DsRed, was localized in the AHN of vGAT-IRES-Cre mice.

(C) Example micrographs showing the expression of EGFP (green) and DsRed (red) at the injection site within the AHN. Note the dually-labeled cells indicate starter cells.

(D) Example micrographs showing DsRed+ cells in different brain regions, including the lateral parabrachial nucleus (LPB) (D1), the paraventricular thalamic nucleus (PVT) (D2), the ventromedial hypothalamic nucleus (VMH) / dorsomedial hypothalamic nucleus (DM) / medial amygdaloid nucleus (MA) (D3), the lateral septum (LS) / medial preoptic area (MPA) (D4), the dorsal part of premammillary nucleus (PMD) / ventral part of premammillary nucleus (PMV) / posterior hypothalamic area (PH) (D5), and the subiculum (S) (D6).

(E) Fraction distribution of total DsRed-labeled cells in different brain regions monosynaptically projecting to the vGAT+AHN neurons.

Scale bars are labeled in the graphs. Numbers of mice $(E)$ are indicated in the graphs. Data in $(E)$ are means \pm SEM. 

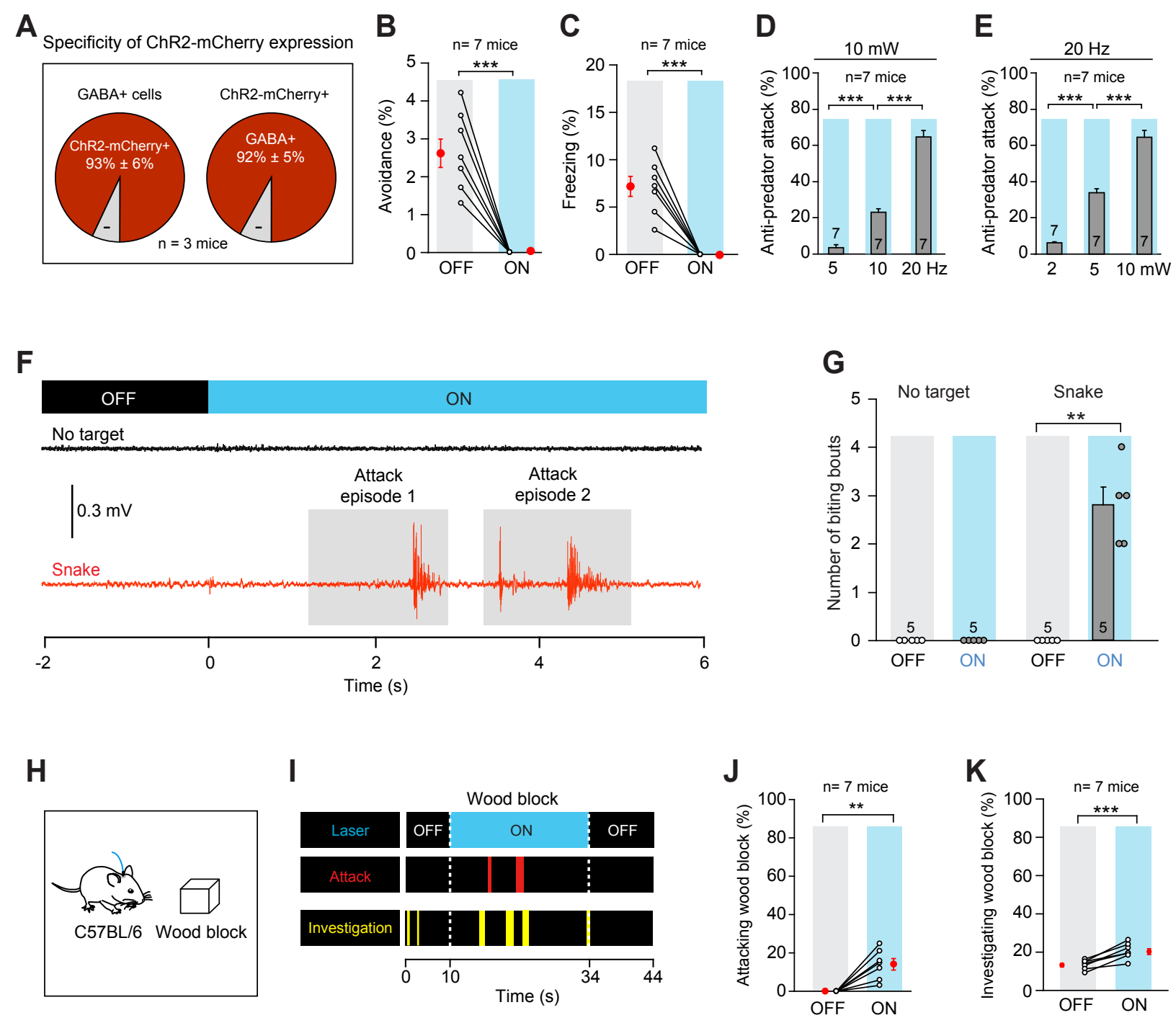

Figure S9 Xie et al., 2020 
Figure S9 Additional quantitative analyses of behaviors evoked by activation of AHN vGAT+ neurons to live snake and wood block. (Related to Figure 4)

(A) Quantitative analyses showing specific expression of ChR2-mCherry in GABA+AHN neurons. For the example micrographs, see Figure 4B.

(B, C) Quantitative analyses of time spent for avoidance (B) and freezing (C) with (ON) and without (OFF) light-stimulation of $v G A T+A H N$ neurons.

(D, E) Quantitative analyses of time spent for anti-predator attack evoked by light-stimulation with different frequencies (D) and with different laser powers (E) on AHN vGAT+ neurons.

(F) Example EMG traces of masseter muscles before (OFF) and during (ON) light stimulation of VGAT+ AHN neurons in the arena without a target (No target) or with a live snake (Snake). Shaded areas indicate the initiation and termination of biting-like attacks based on visual inspection of the video taken by high-speed camera.

(G) Quantitative analyses of biting bouts, as indicated by EMG, evoked by photostimulation of VGAT+ AHN neurons in the arena without a target (No target) or with a live snake (Snake).

(H) Schematic diagram showing the behavioral paradigm to measure mouse behaviors to a wood block in an enclosed arena.

(I) Behavioral ethogram of an example mouse to the wood block in the arena before, during and after light stimulation of AHN vGAT+ neurons. The colored bars in the ethograms indicated the onset and offset of specific behaviors.

$(\mathrm{J}, \mathrm{K})$ Quantitative analyses of time spent for attacking $(\mathrm{J})$ and investigating $(\mathrm{K})$ the wood block before (OFF) and during (ON) light stimulation of AHN vGAT+ neurons.

Scale bars are labeled in the graphs. Numbers of mice are indicated in the graphs (A-E, $G, J, K)$. Data in (A-E, G, J, K) are means \pm SEM. For the $P$ values, see Table S4. 
A

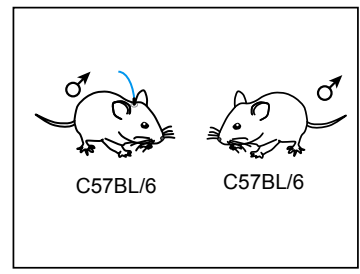

F

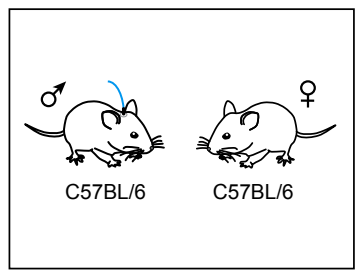

B

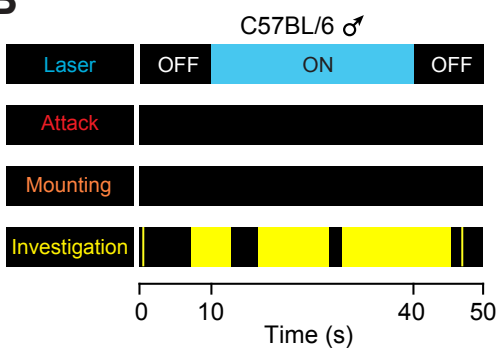

G

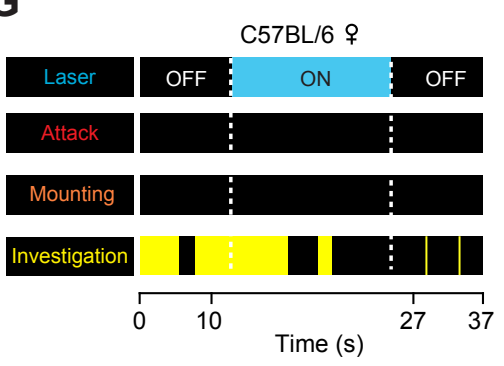

C

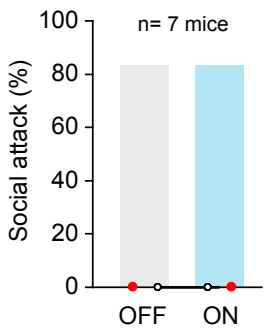

H

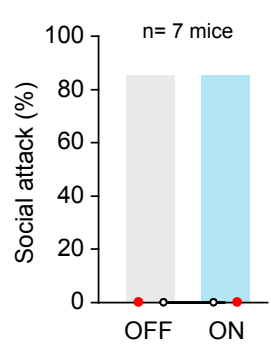

D

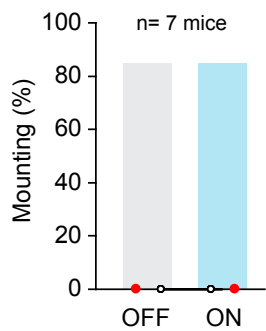

I

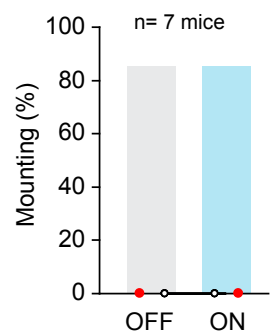

E

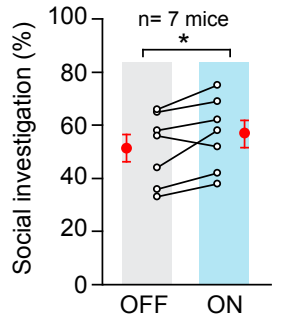

J

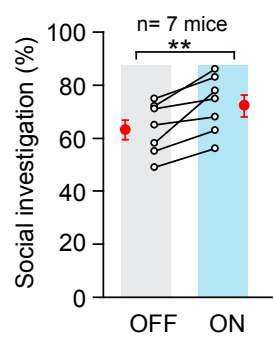

Figure S10 Xie et al., 2020 
Figure S10 Additional quantitative analyses of behaviors evoked by activation of vGAT+ AHN neurons to conspecifics. (Related to Figure 4)

(A, F) Schematic diagrams showing the behavioral paradigm to measure mouse behaviors to male $(A)$ and female conspecifics $(F)$ in an enclosed arena.

(B, G) Behavioral ethograms of example mice to male (B) and female (G) conspecifics before, during and after photostimulation of AHN vGAT+ neurons. The colored bars in the ethograms indicated the onset and offset of specific behaviors.

(C, H) Quantitative analyses of time spent for social attack toward male (C) and female $(H)$ conspecifics before (OFF) and during (ON) photostimulation of $v G A T+A H N$ neurons. (D, I) Quantitative analyses of time spent for mounting on male (D) and female (I) conspecifics before (OFF) and during (ON) photostimulation of VGAT+ AHN neurons.

(E, J) Quantitative analyses of time spent for social investigation of male (E) and female $(\mathrm{J})$ conspecifics before (OFF) and during (ON) photostimulation of $v G A T+A H N$ neurons.

Numbers of mice are indicated in the graphs (C-E, H-J). Data in (C-E, H-J) are means \pm SEM. Statistical analyses were performed by Student $t$-tests $\left({ }^{*}, \mathrm{P}<0.05 ;{ }^{* *}, \mathrm{P}<0.01\right)$. For the $P$ values, see Table S4. 
A

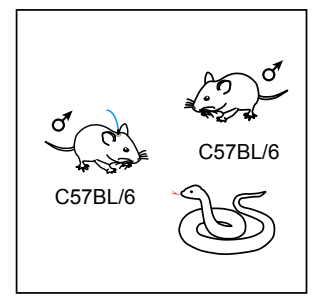

E

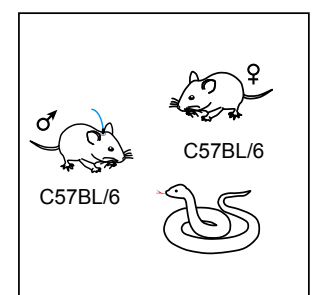

B

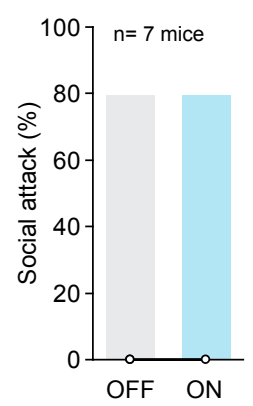

F

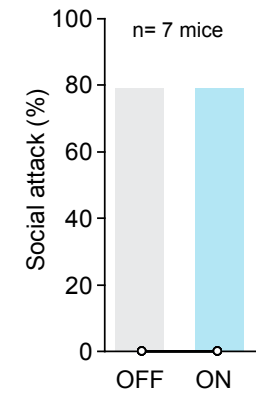

C

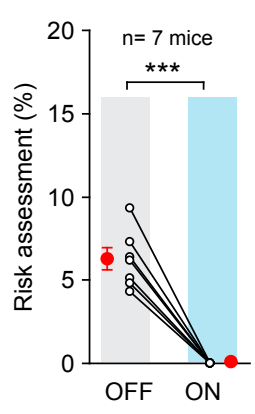

G

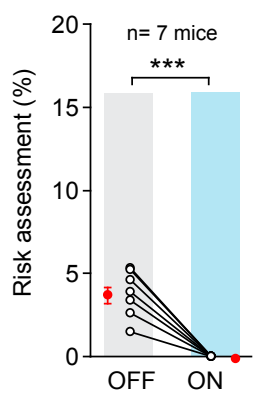

D

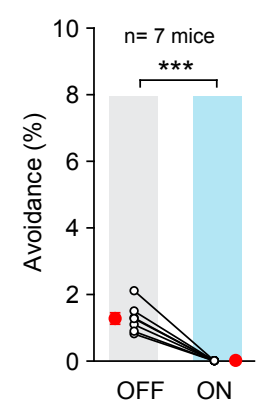

H

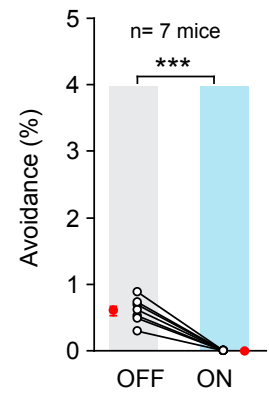

Figure S11 Xie et al., 2020 
Figure S11 Additional quantitative analyses of behaviors evoked by activation of VGAT+ AHN neurons to live snake and conspecifics. (Related to Figure 4)

(A, E) Schematic diagrams showing the behavioral paradigm to measure mouse behaviors to live snake versus male conspecifics (A) or live snake versus female conspecifics $(H)$ in an enclosed arena.

(B, F) Quantitative analyses of time spent for social attack toward male (B) or female (F) conspecifics before (OFF) and during (ON) photostimulation of AHN vGAT+ neurons.

(C, G) Quantitative analyses of time spent for risk assessment toward live snake before (OFF) and during (ON) photostimulation of AHN vGAT+ neurons in the presence of male (C) or female (G) conspecifics.

(D, H) Quantitative analyses of time spent for avoidance from live snake before (OFF) and during (ON) photostimulation of AHN vGAT+ neurons in the presence of male (D) and female $(\mathrm{H})$ conspecifics.

Numbers of mice are indicated in the graphs (B-D, F-H). Data in (B-D, F-H) are means \pm SEM (error bars). Statistical analyses were performed by Student $t$-tests $\left({ }^{* * *}, \mathrm{P}<0.001\right)$. For the $\mathrm{P}$ values, see Table S4. 
A

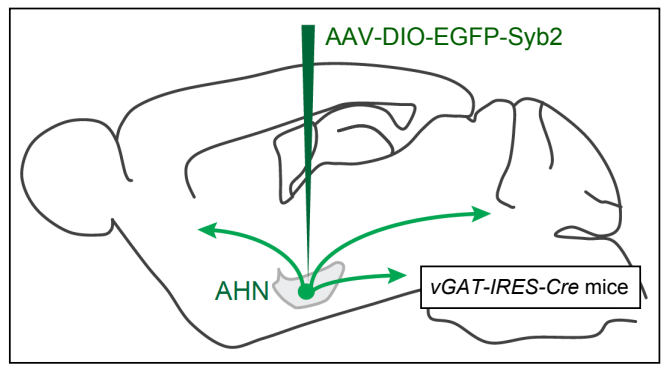

C

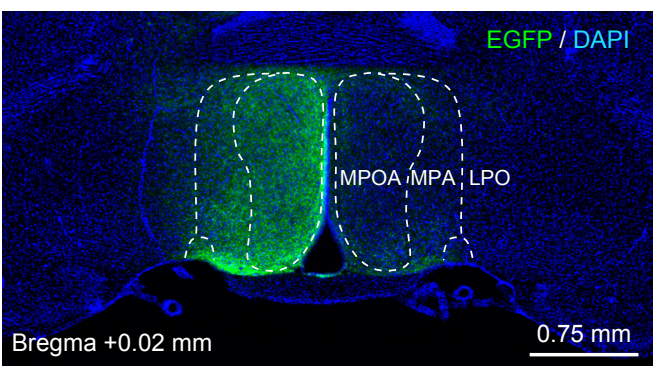

D

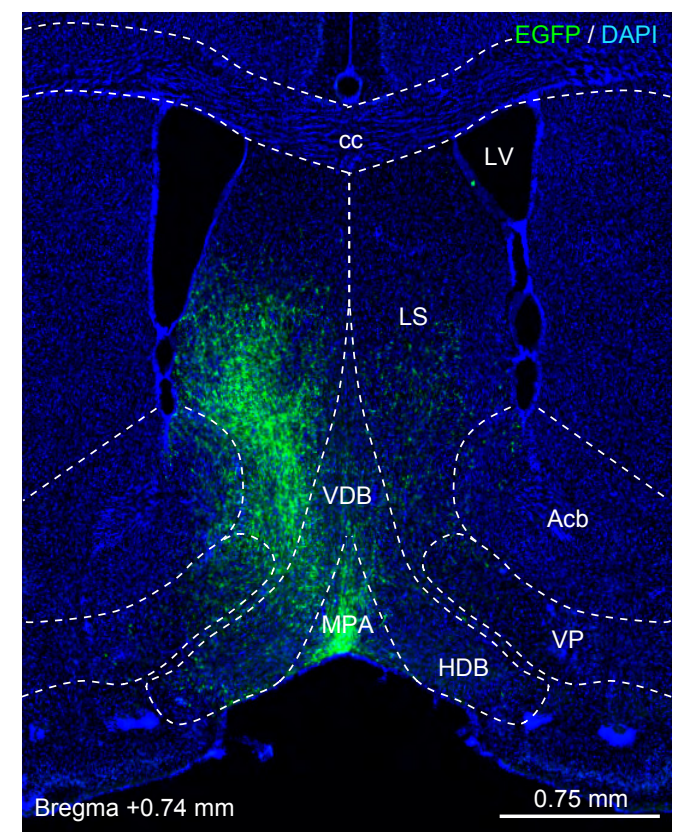

B

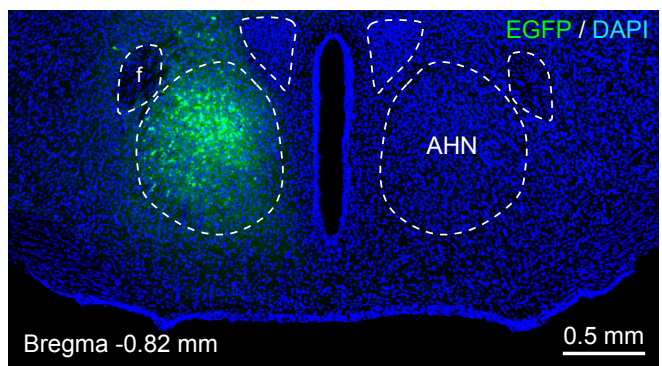

E

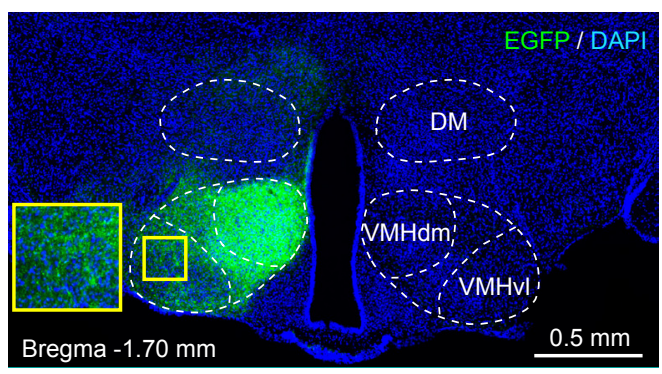

F

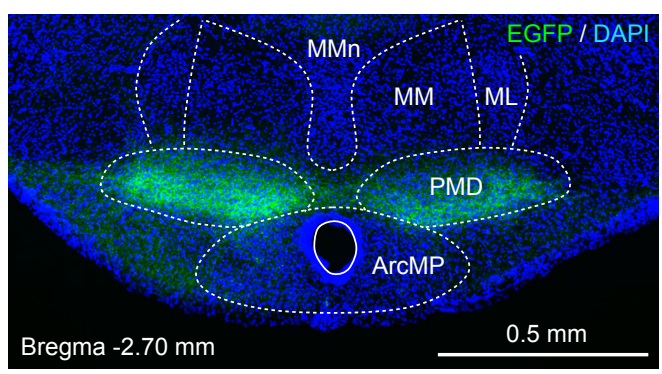

G

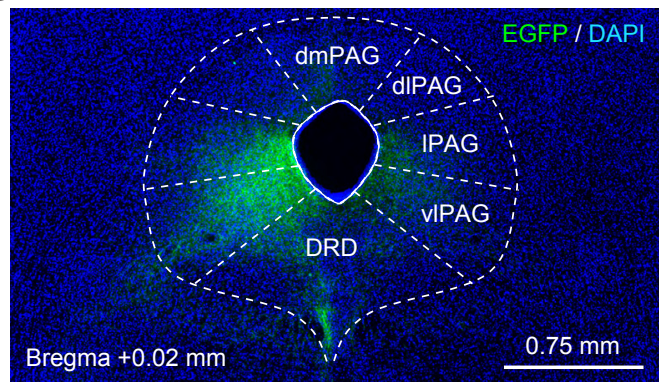

Figure S12 Xie et al., 2020 
Figure S12 Efferent projections of AHN vGAT+ neurons. (Related to Figure 5)

195 (A) Schematic diagram showing the strategy to map the efferent projections of AHN 196 vGAT+ neurons.

197 (B) Example coronal section of vGAT-IRES-Cre mice showing the injection center of 198 AAV-DIO-EGFP-Syb2 with fluorescence signals of EGFP-Syb2 restricted within the 199 AHN.

200 (C-G) Example micrographs showing EGFP+ synaptic terminals of AHN vGAT+ neurons 201 in the target brain regions, including MPOA/MPA (C), LS (D), VMH (E), PMD (F), and 202 PAG (G). Inset in (E), modest EGFP-Syb2 signals in the VMHvl, suggesting AHN vGAT+ 203 neurons may exert GABAergic inhibition to neurons in the VMHvl. Scale bars are labeled 204 in the graphs. 
A

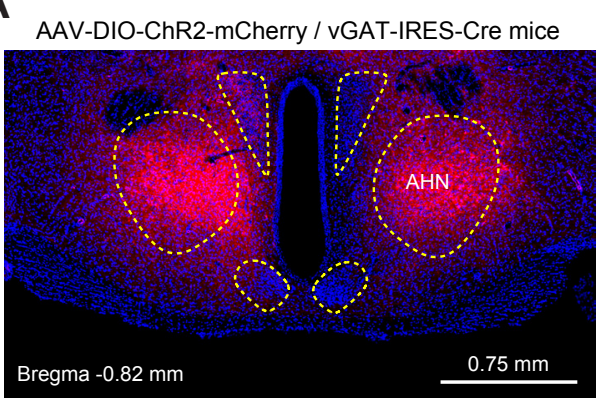

C

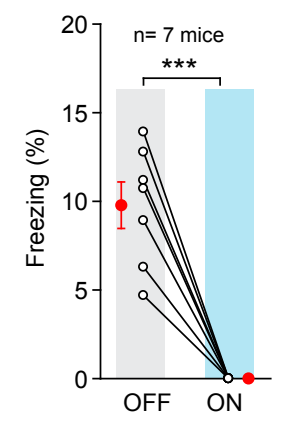

B

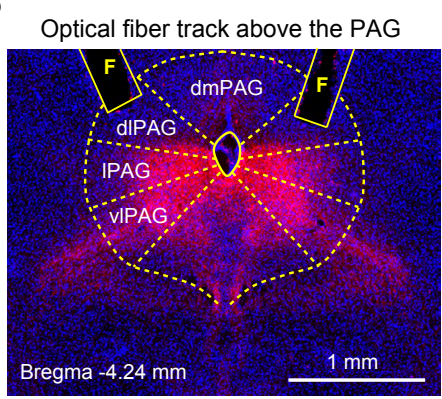

E

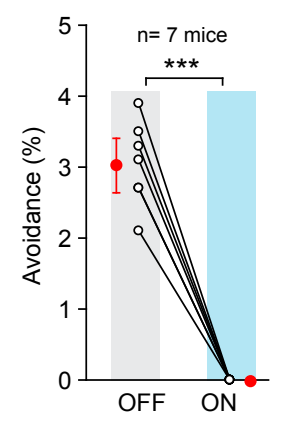

Figure S13 Xie et al., 2020 
Figure S13 Activation of vGAT+ AHN-PAG pathway. (Related to Figure 5)

206 (A) An example coronal section showing that ChR2-mCherry expression is largely restricted within the AHN of VGAT-IRES-Cre mice.

208 (B) An example coronal section showing the optical-fiber tracks above the 209 ChR2-mCherry+ axon terminals in the vIPAG.

210 (C-E) Quantitative analyses of time spent for freezing (C), risk assessment toward snake 211 (D), and avoidance from snake (E) of mice before (OFF) and during (ON) 212 photostimulation of vGAT+ AHN-PAG pathway.

213 Scale bars are labeled in the graphs. Numbers of mice are indicated in the graphs (C-E). 214 Data in (C-E) are means \pm SEM (error bars). Statistical analyses were performed by 215 Student $t$-tests (***, $\mathrm{P}<0.001)$. For the $\mathrm{P}$ values, see Table S4. 
A

Activation of VGAT+ AHN-LS pathway

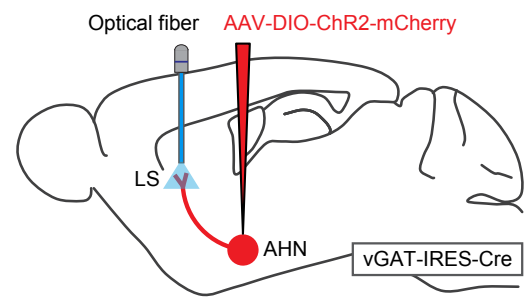

D

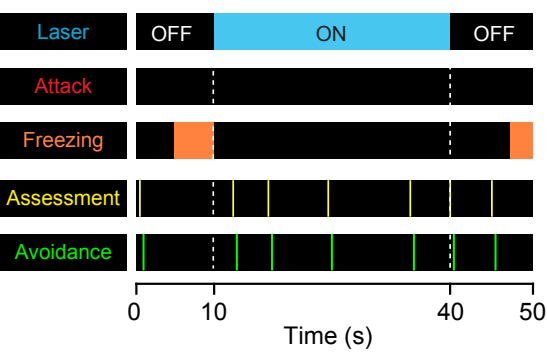

B

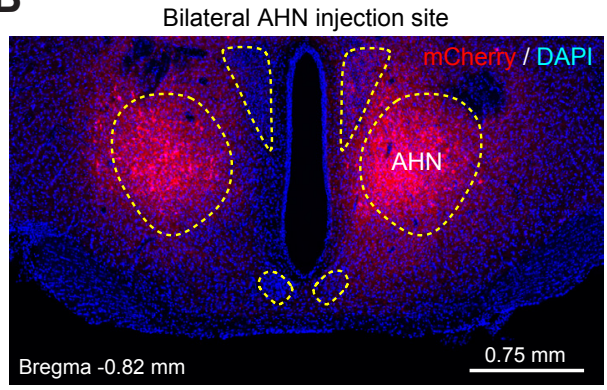

C

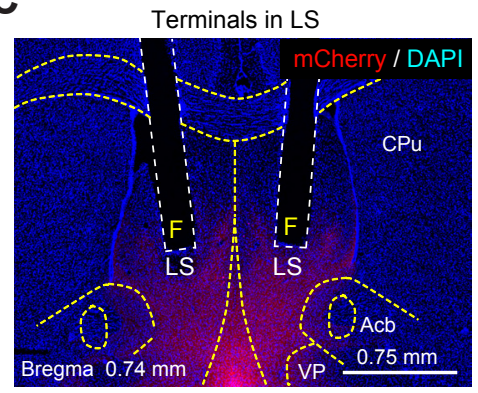

G

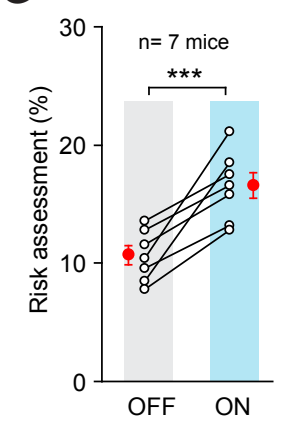

H

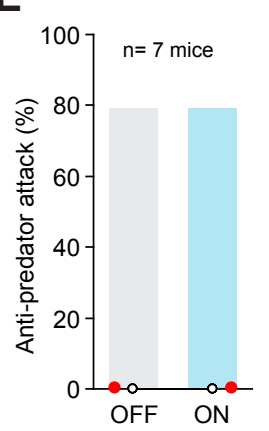

F
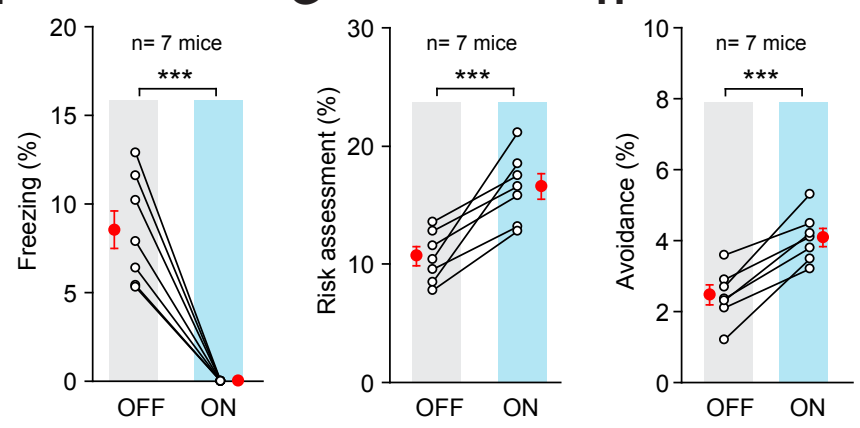

Figure S14 Xie et al., 2020 
217 (A) Schematic diagram showing AAV-DIO-ChR2-mCherry injection into the AHN of vGAT-IRES-Cre mice, and optic fiber implantation above the axon terminals of AHN vGAT+ neurons for light stimulation.

(B) An example coronal section showing ChR2-mCherry expression largely restricted within the AHN of vGAT-IRES-Cre mice.

(C) An example coronal section showing the optical-fiber tracks above the ChR2-mCherry+ axon terminals in the LS.

(D) Behavioral ethogram of an example mouse before, during, and after photostimulation of vGAT+ AHN-LS pathway. The colored bars in the ethograms indicated the onset and offset of specific behaviors.

(E-H) Quantitative analyses of time spent for anti-predator attack $(E)$, freezing $(F)$, risk assessment to snake $(G)$, and avoidance from snake $(H)$ of mice before (OFF) and during (ON) photostimulation of vGAT+ AHN-LS pathway.

Scale bars are labeled in the graphs. Numbers of mice are indicated in the graphs $(\mathrm{E}-\mathrm{H})$. Data in $(E-H)$ are means \pm SEM (error bars). Statistical analyses were performed by Student $t$-tests $\left({ }^{* *} \mathrm{P}<0.001\right)$. For the $\mathrm{P}$ values, see Table S4. 

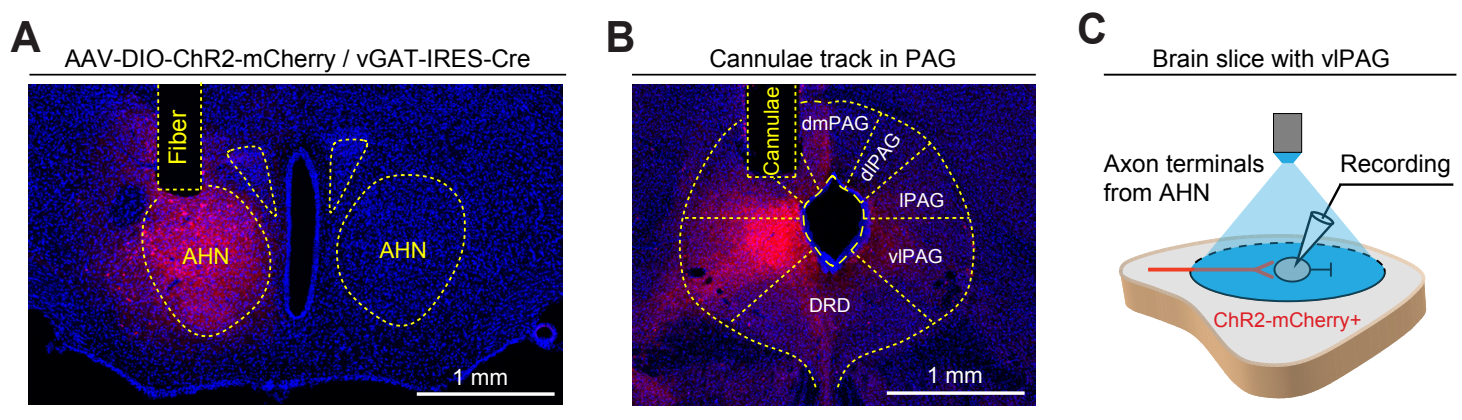

D

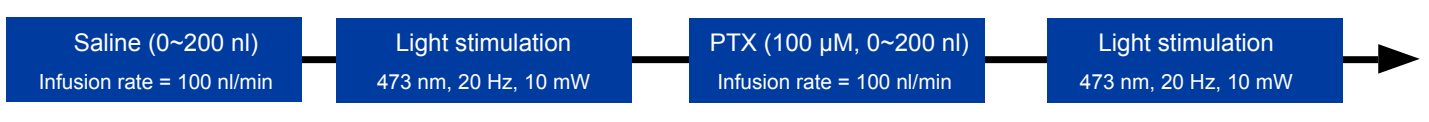

E

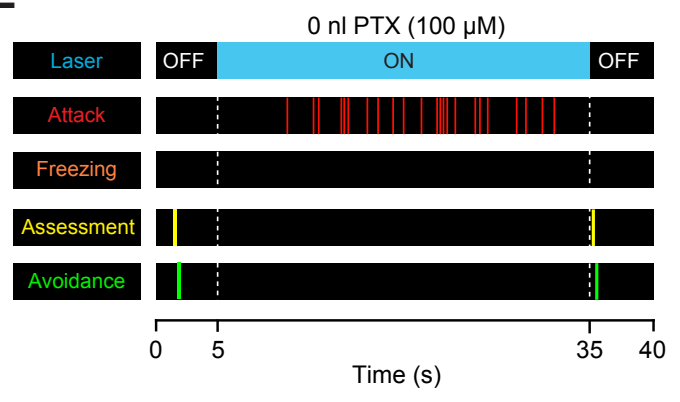

$\mathbf{F}$

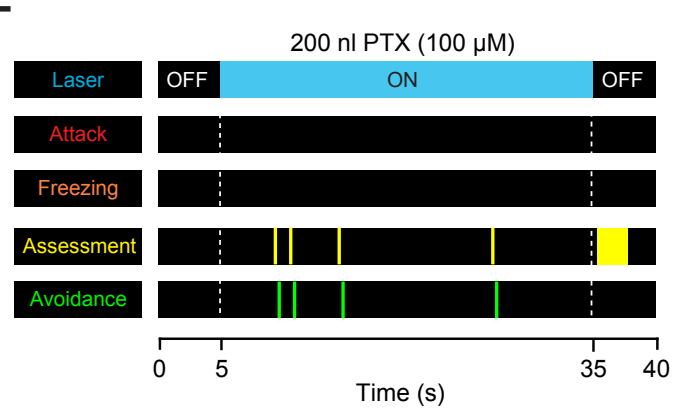

Figure S15 Xie et al., 2020 
Figure S15 Effect of picrotoxin infused into the vIPAG on the anti-predator attack evoked by activation of AHN vGAT+ neurons. (Related to Figure 5)

(A) An example coronal section showing ChR2-mCherry expression largely restricted within the AHN of vGAT-IRES-Cre mice and an optical fiber track above the AHN.

(B) An example coronal section showing the ChR2-mCherry+ axon terminals in the VIPAG and the cannulae track above these axon terminals.

(C) Schematic diagram showing whole-cell recording of light-evoked GABAergic postsynaptic currents from the vIPAG neurons in acute brain slices.

(D) Schematic diagram showing the procedure for infusing saline and picrotoxin $(100 \mu \mathrm{M})$ into the VIPAG combined with measuring anti-predator attack behavior evoked by activation of vGAT+ AHN neurons.

(E, F) Behavioral ethograms of an example mouse infused with $0 \mathrm{nl}$ PTX (E) and $200 \mathrm{nl}$ PTX (F) into the vIPAG before, during and after light stimulation of AHN vGAT+ neurons in the arena with a live snake. The colored bars in the ethograms indicated the onset and offset of specific behaviors. 
A

AAV-DIO-GtACR1-2A-EGFP / vGAT-IRES-Cre

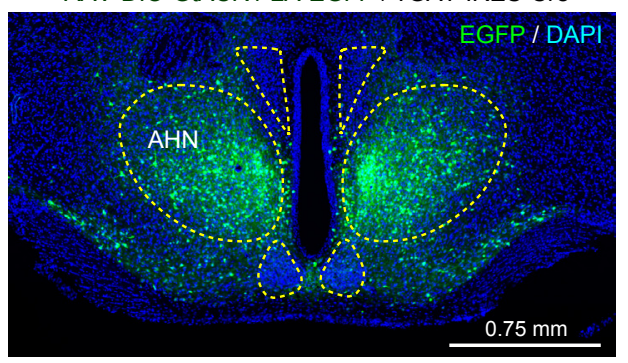

B

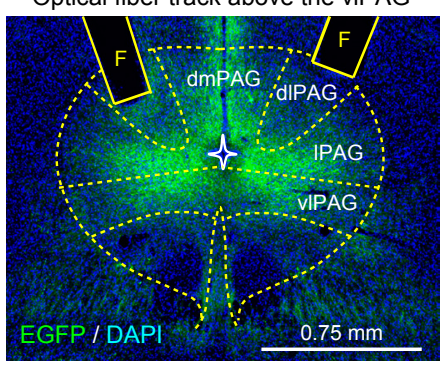

C Photoinhibition of vGAT+ AHN-PAG pathway

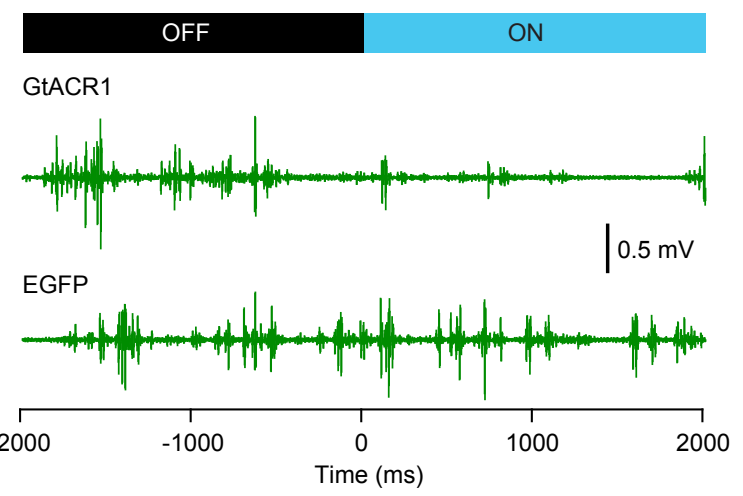

Figure S16 Xie et al., 2020 
248 Figure S16 Photoinhibition of vGAT+ AHN-vIPAG pathway impaired 249 mechanically-evoked defensive attack. (Related to Figure 5)

250 (A) Example coronal section showing expression of EGFP and GtACR1 largely restricted 251 within the AHN of VGAT-IRES-Cre mice.

252 (B) Example micrograph showing two optical-fiber tracks above the EGFP+ axon 253 terminals in the vIPAG.

254 (C) Example traces of EMG recorded from masseter muscles of mice with (GtACR1) and 255 without (EGFP) photoinhibition of VGAT+AHN-PAG pathway.

256 Scale bars were indicated in the graphs. 
Movie S1 Jumping escape and defensive attack of a male C57BL/6 mouse in the mouse-versus-snake paradigm.

Movie S2 Defensive attack of a Wistar rat in the rat-versus-snake paradigm.

Movie S3 Defensive attack of a greater long-tailed hamster (Tscherskia triton) in the hamster-versus-snake paradigm.

Movie S4 Behavioral responses of a male C57BL/6 mouse to a dummy snake coated with and without snake feces.

Movie S5 Behavioral responses of a male C57BL/6 mouse to a dummy snake equipped with an alligator-clip to apply noxious mechanical stimuli on the tail.

Movie S6 Behavioral responses of a male C57BL/6 mouse to neutral object equipped with an alligator-clip to apply noxious mechanical stimuli on the tail.

Movie S7 Photoinhibition of AHN vGAT+ neurons of a male C57BL/6 mouse reversibly abrogated mechanically-evoked defensive attack to the dummy snake.

Movie S8 Photostimulation of AHN vGAT+ neurons of a male C57BL/6 mouse evoked biting attack to a live snake in the arena.

Movie S9 Photostimulation of AHN vGAT+ neurons of a male C57BL/6 mouse evoked activity of masseter muscles in parallel with biting attack toward live snake.

Movie S10 Photostimulation of AHN vGAT+ neurons of a male C57BL/6 mouse evoked biting attack to a wood block in the arena.

Movie S11 Photostimulation of AHN vGAT+ neurons of a male C57BL/6 mouse abrogated its ongoing social aggression against a male intruder.

Movie S12 Photostimulation of vGAT+ AHN neurons of a male C57BL/6 mouse did not evoke biting attack or mounting to another male mouse in the arena. 
280 Movie S13 Photostimulation of vGAT+ AHN neurons of a male C57BL/6 mouse did not evoke biting attack or mounting to a female mouse in the arena.

282 Movie S14 In the presence of both a live snake and a male C57BL/6 mouse, photostimulation of AHN vGAT+ neurons evoked biting attack selectively to the live snake rather than the male C57BL/6 mouse in the arena.

Movie S15 In the presence of both a live snake and a female C57BL/6 mouse, photostimulation of AHN vGAT+ neurons evoked biting attack selectively to the live snake rather than the female $\mathrm{C} 57 \mathrm{BL} / 6$ mouse in the arena.

Table S1 Information of mouse lines and reagents

Table S2 Summary of all experimental designs

Table S3 Summary of cell-counting strategies

Table S4 Summary of statistical analyses 


\section{Table S1 Information of mouse lines and reagents}

\begin{tabular}{|c|c|c|}
\hline \multicolumn{3}{|c|}{ Mouse Lines } \\
\hline vGlut2-IRES-Cre & JAX Mice & Stock No. 028863 \\
\hline vGAT-IRES-Cre & JAX Mice & Stock No. 028862 \\
\hline MrgprD-CreERT2 & JAX Mice & Stock No. 031286 \\
\hline iDTR & JAX Mice & Stock No. 007900 \\
\hline$A i 3$ & JAX Mice & Stock No. 007903 \\
\hline \multicolumn{3}{|c|}{ Antibodies } \\
\hline Anti-EGFP & Abcam & ab290 / ab13970 \\
\hline Anti-mCherry & Abcam & ab167453 / ab205402 \\
\hline Anti-Glutamate & Sigma & G6642 \\
\hline Anti-GABA & Sigma & A2052 \\
\hline \multicolumn{3}{|c|}{ Chemical reagents } \\
\hline D-AP5 / CNQX & Tocris & Cat. No. $0106 / 0190$ \\
\hline Picrotoxin / TTX & Tocris & Cat. No. $1128 / 1078$ \\
\hline 4-AP & Sigma & Cat. No. 275875 \\
\hline DAPI & Sigma & Cat. No. D8417 \\
\hline \multicolumn{3}{|c|}{ AAV vectors } \\
\hline AAV2/9-CAG-DIO-GtACR1-2A-EGFP & Plasmid made by TaiTool Co., Ltd & Packaged by TaiTool Co., Ltd., China \\
\hline AAV2/9-EF1a-DIO-EGFP & Plasmid made by TaiTool Co., Ltd & Packaged by TaiTool Co., Ltd., China \\
\hline AAV2/9-EF1a-DIO-ChR2-mCherry & Plasmid made by TaiTool Co., Ltd & Packaged by TaiTool Co., Ltd., China \\
\hline AAV2/9-EF1a-DIO-mCherry & Plasmid made by TaiTool Co., Ltd & Packaged by TaiTool Co., Ltd., China \\
\hline AAV2/9-EF1a-DIO-jGCaMP7s & Plasmid made by TaiTool Co., Ltd & Packaged by TaiTool Co., Ltd., China \\
\hline AAV2/9-EF1a-DIO-EGFP-Syb2 & Plasmid made by TaiTool Co., Ltd & Packaged by TaiTool Co., Ltd., China \\
\hline AAV2/9-EF1a-DIO-EGFP-2A-TVA & Plasmid made by BrainVTA Co., Ltd. & Produced by BrainVTA Co., Ltd.,China \\
\hline AAV2/9-EF1a-DIO-RV-G & Plasmid made by BrainVTA Co., Ltd. & Produced by BrainVTA Co., Ltd.,China \\
\hline RV-EnvA- $\Delta$ G-DsRed & Plasmid made by BrainVTA Co., Ltd. & Produced by BrainVTA Co., Ltd.,China \\
\hline
\end{tabular}




\section{Table S2 Summary of all experimental designs}

\begin{tabular}{|c|c|c|c|c|}
\hline Figures & Aims & Mouse lines & AAV injection \& optical fiber implantation & Type of data \\
\hline Figure S1 & $\begin{array}{l}\text { Measuring defensive attack in } \\
\text { rodent-versus-snake paradigms }\end{array}$ & $\begin{array}{l}\text { Mice, Rats, } \\
\text { Hamsters }\end{array}$ & No AAV injection. No optical fiber implantation & Behavior \\
\hline $\begin{array}{l}\text { Figure } 1 \mathrm{~A}-1 \mathrm{~F} \\
\text { Figure } \mathrm{S} 2\end{array}$ & $\begin{array}{l}\text { Analyzing sensory-triggered } \\
\text { defensive attack }\end{array}$ & WT mice & No AAV injection. No optical fiber implantation & Behavior \\
\hline Figure S3 & $\begin{array}{l}\text { Defensive attack to neutral object } \\
\text { linked to noxious mechanical stimuli }\end{array}$ & WT mice & No AAV injection. No optical fiber implantation & Behavior \\
\hline Figure 1G-1I & $\begin{array}{l}\text { Effect of ablation of MrgprD+ neurons on } \\
\text { mechanically-evoked defensive attack }\end{array}$ & $\begin{array}{l}\text { MrgprD-CreERT2; } \\
\text { iDTR; Ai3 mice }\end{array}$ & No AAV injection. No optical fiber implantation & $\begin{array}{l}\text { Histology } \\
\text { Behavior }\end{array}$ \\
\hline Figure S4A-S4C & Cell-type specificity of AHN neurons & WT mice & No AAV injection. No optical fiber implantation & Histology \\
\hline Figure S4D-S4O & $\begin{array}{l}\text { Specificity of vGAT-IRES-Cre and } \\
\text { vGlut2-IRES-Cre mice }\end{array}$ & $\begin{array}{l}\text { vGAT-IRES-Cre } \\
\text { vGlut2-IRES-Cre }\end{array}$ & $\begin{array}{l}\text { AAV-DIO-EGFP injected into the AHN of } \\
\text { vGAT-IRES-Cre or vGlut2-IRES-Cre mice }\end{array}$ & Histology \\
\hline $\begin{array}{l}\text { Figure } 2 \\
\text { Figure S5 }\end{array}$ & $\begin{array}{l}\text { Effect of photoinhibition of AHN vGAT+ } \\
\text { or vGlut } 2+\text { neurons on mechanically- } \\
\text { evoked defensive attack }\end{array}$ & $\begin{array}{l}\text { vGAT-IRES-Cre } \\
\text { vGlut2-IRES-Cre }\end{array}$ & $\begin{array}{l}\text { AAV-DIO-GtACR1-2A-EGFP or AAV-DIO-EGFP injected } \\
\text { into the AHN of VGAT-IRES-Cre or vGlut2-IRES-Cre mice } \\
\text { Optical fibers implanted above the AHN bilaterally }\end{array}$ & $\begin{array}{l}\text { Histology } \\
\text { Slice physiology } \\
\text { EMG / Behavior }\end{array}$ \\
\hline $\begin{array}{l}\text { Figure } 3 A-3 C \\
\text { Figure S6 }\end{array}$ & $\begin{array}{l}\text { Fiber photometry recording from } \\
\text { AHN vGAT+ neurons }\end{array}$ & vGAT-IRES-Cre & $\begin{array}{l}\text { AAV-DIO-GCaMP7s injected into the AHN of } \\
\text { VGAT-IRES-Cre mice } \\
\text { Optical fiber implanted above the AHN }\end{array}$ & $\begin{array}{l}\text { Histology } \\
\text { Fiber photometry }\end{array}$ \\
\hline $\begin{array}{l}\text { Figure 3D-3L } \\
\text { Figure S7 }\end{array}$ & $\begin{array}{l}\text { Single-unit recording from AHN } \\
\text { vGAT+ neurons }\end{array}$ & vGAT-IRES-Cre & $\begin{array}{l}\text { AAV-DIO-ChR2-mCherry injected into the AHN of } \\
\text { VGAT-IRES-Cre mice } \\
\text { Optrode inserted into the AHN for single-unit recording }\end{array}$ & $\begin{array}{l}\text { Histology } \\
\text { Single-unit recording }\end{array}$ \\
\hline Figure S8 & $\mathrm{RV}$-mediated retrograde tracing & vGAT-IRES-Cre & See Figure S8A & Histology \\
\hline $\begin{array}{l}\text { Figure } \mathbf{4 A - 4 G} \\
\text { Figure S9 }\end{array}$ & $\begin{array}{l}\text { Effect of light stimulation of AHN } \\
\text { vGAT+ neurons on anti-predator } \\
\text { defensive behaviors }\end{array}$ & vGAT-IRES-Cre & $\begin{array}{l}\text { AAV-DIO-ChR2-mCherry injected into the AHN of } \\
\text { vGAT-IRES-Cre mice } \\
\text { Optical fiber implanted above the AHN }\end{array}$ & $\begin{array}{l}\text { Histology } \\
\text { Slice physiology } \\
\text { EMG / Behavior }\end{array}$ \\
\hline $\begin{array}{l}\text { Figure } 4 \mathrm{H}-\mathbf{4 K} \\
\text { Figure } \mathrm{S} 10\end{array}$ & $\begin{array}{l}\text { Effect of light stimulation of AHN } \\
\text { vGAT+ neurons on social behaviors }\end{array}$ & vGAT-IRES-Cre & $\begin{array}{l}\text { AAV-DIO-ChR2-mCherry injected into the AHN of } \\
\text { vGAT-IRES-Cre mice } \\
\text { Optical fiber implanted above the AHN }\end{array}$ & Behavior \\
\hline $\begin{array}{l}\text { Figure 4L-4S } \\
\text { Figure S11 }\end{array}$ & $\begin{array}{l}\text { Effect of light stimulation of AHN } \\
\text { vGAT+ neurons in a two-target paradigm }\end{array}$ & vGAT-IRES-Cre & $\begin{array}{l}\text { AAV-DIO-ChR2-mCherry injected into the AHN of } \\
\text { VGAT-IRES-Cre mice } \\
\text { Optical fiber implanted above the AHN }\end{array}$ & Behavior \\
\hline Figure S12 & Efferents of AHN vGAT+ neurons & vGAT-IRES-Cre & $\begin{array}{l}\text { AAV-DIO-EGFP-Syb2 injected into the AHN of } \\
\text { vGAT-IRES-Cre mice }\end{array}$ & Histology \\
\hline $\begin{array}{l}\text { Figure } \mathbf{5 A - 5 C} \\
\text { Figure S13 } \\
\text { Figure } \mathrm{S} 14\end{array}$ & $\begin{array}{l}\text { Effects of activation of vGAT+ } \\
\text { AHN-LS and AHN-vIPAG pathway }\end{array}$ & vGAT-IRES-Cre & $\begin{array}{l}\text { AAV-DIO-ChR2-mCherry injected into the AHN of } \\
\text { vGAT-IRES-Cre mice } \\
\text { Optical fiber implanted above the vIPAG or LS }\end{array}$ & $\begin{array}{l}\text { Histology } \\
\text { Behavior }\end{array}$ \\
\hline $\begin{array}{l}\text { Figure 5D-5H } \\
\text { Figure S15 }\end{array}$ & $\begin{array}{l}\text { Effects of PTX infusion to vIPAG on } \\
\text { light-evoked anti-predator attack }\end{array}$ & vGAT-IRES-Cre & $\begin{array}{l}\text { AAV-DIO-ChR2-mCherry injected into the AHN of } \\
\text { vGAT-IRES-Cre mice } \\
\text { Optical fiber implanted above the AHN } \\
\text { Cannulae implanted above the vIPAG }\end{array}$ & $\begin{array}{l}\text { Histology } \\
\text { Slice physiology } \\
\text { Behavior }\end{array}$ \\
\hline $\begin{array}{l}\text { Figure 5I-5K } \\
\text { Figure S16 }\end{array}$ & $\begin{array}{l}\text { Effects of photoinhibition of vGAT+ } \\
\text { AHN-vIPAG pathway on mechanically- } \\
\text { evoked defensive attack behavior }\end{array}$ & $v G A T-I R E S-C r e$ & $\begin{array}{l}\text { AAV-DIO-GtACR1-2A-EGFP injected into the AHN of } \\
\text { vGAT-IRES-Cre mice } \\
\text { Optical fiber implanted above the vIPAG }\end{array}$ & $\begin{array}{l}\text { Histology } \\
\text { EMG / Behavior }\end{array}$ \\
\hline
\end{tabular}


Table S3 Summary of cell-counting strategies

\begin{tabular}{|c|c|c|c|c|}
\hline Brain region & Section Type & Section Range & Total collection & Sampling \\
\hline LS & Coronal section $(40 \mu \mathrm{m})$ & Bregma $(+1.54$ to -0.10$)$ & Approximately 41 sections & To sample all the sections \\
\hline MPA & Coronal section $(40 \mu \mathrm{m})$ & Bregma $(+0.74$ to -0.58$)$ & Approximately 33 sections & To sample all the sections \\
\hline $\mathrm{PVH}$ & Coronal section $(40 \mu \mathrm{m})$ & Bregma $(-0.58$ to -1.22$)$ & Approximately 16 sections & To sample all the sections \\
\hline so & Coronal section $(40 \mu \mathrm{m})$ & Bregma $(-0.58$ to -0.94$)$ & Approximately 9 sections & To sample all the sections \\
\hline VMH & Coronal section $(40 \mu \mathrm{m})$ & Bregma (-1.06 to -2.06$)$ & Approximately 25 sections & To sample all the sections \\
\hline DM & Coronal section $(40 \mu \mathrm{m})$ & Bregma (-1.34 to -2.18$)$ & Approximately 21 sections & To sample all the sections \\
\hline MA & Coronal section $(40 \mu \mathrm{m})$ & Bregma $(-0.94$ to -2.18$)$ & Approximately 31 sections & To sample all the sections \\
\hline PVT & Coronal section $(40 \mu \mathrm{m})$ & Bregma $(-0.22$ to -2.18$)$ & Approximately 49 sections & To sample all the sections \\
\hline PMD & Coronal section $(40 \mu \mathrm{m})$ & Bregma $(-2.46$ to -2.70$)$ & Approximately 6 sections & To sample all the sections \\
\hline PMV & Coronal section $(40 \mu \mathrm{m})$ & Bregma $(-2.30$ to -2.54$)$ & Approximately 6 sections & To sample all the sections \\
\hline $\mathrm{PH}$ & Coronal section $(40 \mu \mathrm{m})$ & Bregma (-1.82 to -2.70$)$ & Approximately 22 sections & To sample all the sections \\
\hline S & Coronal section $(40 \mu \mathrm{m})$ & Bregma (-2.46 to -4.36$)$ & Approximately 47 sections & To sample all the sections \\
\hline $\mathrm{AHi}$ & Coronal section $(40 \mu \mathrm{m})$ & Bregma $(-1.94$ to -3.80$)$ & Approximately 47 sections & To sample all the sections \\
\hline LPB & Coronal section $(40 \mu \mathrm{m})$ & Bregma $(-4.96$ to -5.68$)$ & Approximately 18 sections & To sample all the sections \\
\hline
\end{tabular}




\section{Table S4 - 1 Summary of statistical analyses}

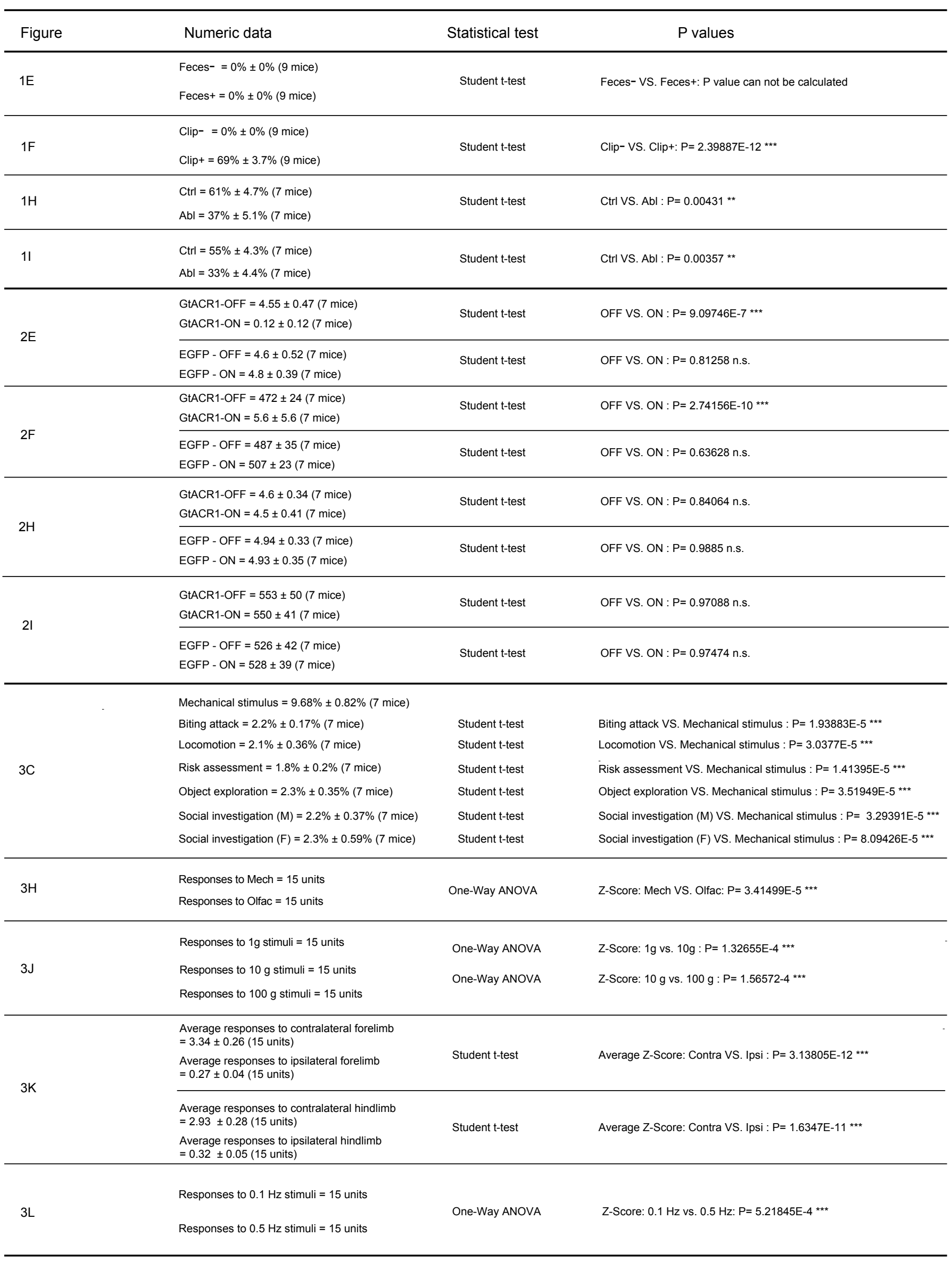




\section{Table S4 - 2 Summary of statistical analyses}

\begin{tabular}{|c|c|c|c|}
\hline Figure & Sample size (n) & Statistical test & $P$ values \\
\hline $4 \mathrm{~F}$ & $\begin{array}{l}\text { ChR2-OFF }=0 \% \pm 0 \%(7 \text { mice }) \\
\text { ChR2-ON }=64.6 \% \pm 3.6 \%(7 \text { mice })\end{array}$ & Paired Student t-test & Anti-predator attack: ON vs. OFF: $\mathrm{P}=4.41921 \mathrm{E}-10$ *** \\
\hline $4 G$ & $\begin{array}{l}\text { ChR2-OFF }=10.1 \% \pm 1.1 \% \text { ( } 7 \text { mice }) \\
\text { ChR2-ON }=0 \% \pm 0 \% \text { (7 mice })\end{array}$ & Paired Student t-test & Risk Assessment: ON vs. OFF: $\mathrm{P}=7.55514 \mathrm{E}-5^{\star \star \star *}$ \\
\hline $4 \mathrm{~J}$ & $\begin{array}{l}\text { ChR2-OFF }=32.4 \% \pm 3 \% \text { (7 mice }) \\
\text { ChR2-ON }=4.1 \% \pm 0.6 \% \text { (7 mice })\end{array}$ & Paired Student t-test & Social attack: ON vs. OFF: $\mathrm{P}=8.50072 \mathrm{E}-7^{* * *}$ \\
\hline $4 \mathrm{~K}$ & $\begin{array}{l}\text { ChR2-OFF }=3.7 \% \pm 0.6 \% \text { (7 mice }) \\
\text { ChR2-ON }=42 \% \pm 4.3 \% \text { (7 mice })\end{array}$ & Paired Student t-test & Social investigation: ON vs. OFF: $\mathrm{P}=1.28897 \mathrm{E}-6^{\star \star \star}$ \\
\hline $4 \mathrm{~N}$ & $\begin{array}{l}\text { ChR2-OFF }=0 \% \pm 0 \% \text { (7 mice }) \\
\text { ChR2-ON }=74.4 \% \pm 4.5 \% \text { (7 mice })\end{array}$ & Paired Student t-test & Anti-predator attack: ON vs. OFF: $\mathrm{P}=1.24154 \mathrm{E}-9^{* * *}$ \\
\hline 40 & $\begin{array}{l}\text { ChR2-OFF }=59.4 \% \pm 3.2 \% \text { (7 mice }) \\
\text { ChR2-ON }=9.5 \% \pm 0.7 \% \text { (7 mice })\end{array}$ & Paired Student t-test & Social investigation: ON vs. OFF: $P=3.33058 E-9 * \star *$ \\
\hline $4 \mathrm{R}$ & $\begin{array}{l}\text { ChR2-OFF }=0 \% \pm 0 \%(7 \text { mice }) \\
\text { ChR2-ON }=67.7 \% \pm 3.6 \% \text { (7 mice })\end{array}$ & Paired Student t-test & Anti-predator attack: ON vs. OFF: $\mathrm{P}=3.07553 \mathrm{E}-10 * * *$ \\
\hline $4 S$ & $\begin{array}{l}\text { ChR2-OFF }=71.3 \% \pm 3 \%(7 \text { mice }) \\
\text { ChR2-ON }=11.2 \% \pm 1.1 \% \text { (7 mice })\end{array}$ & Paired Student t-test & Social investigation: ON vs. OFF: $\mathrm{P}=2.66564 \mathrm{E}-10^{* \star *}$ \\
\hline $5 \mathrm{C}$ & $\begin{array}{l}\text { ChR2-OFF }=0 \% \pm 0 \%(7 \text { mice }) \\
\text { ChR2-ON }=59.7 \% \pm 5.5 \%(7 \text { mice })\end{array}$ & Paired Student t-test & Anti-predator attack: ON vs. OFF: $\mathrm{P}=1.34613 \mathrm{E}-7^{* \star *}$ \\
\hline $5 \mathrm{~F}$ & $\begin{array}{l}\text { Before }=0.93 \pm 0.09 \mathrm{nA} \\
\mathrm{APV}+\mathrm{CNQX}=0.88 \pm 0.08 \mathrm{nA} \\
\mathrm{PTX}=0.04 \pm 0.01 \mathrm{nA}\end{array}$ & $\begin{array}{l}\text { Paired Student t-test } \\
\text { Paired Student t-test }\end{array}$ & $\begin{array}{l}\text { IPSC amplitude: } A P V+C N Q X \text { vs. Before: } P=0.64586 \text {, n.s. } \\
\text { IPSC amplitude: PTX vs. Before: } P=1.31649 E-6 \text { *** }\end{array}$ \\
\hline $5 G$ & $\begin{array}{l}\text { OFF: } 0 \mathrm{nl} \mathrm{PTX}=0 \% \pm 0 \% \text { (6 mice }) \\
\text { OFF: } 100 \mathrm{nl} \mathrm{PTX}=0 \% \pm 0 \% \text { (6 mice }) \\
\text { OFF: } 200 \mathrm{nl} \mathrm{PTX}=0 \% \pm 0 \% \text { (6 mice }) \\
\text { ON: } 0 \mathrm{nl} \mathrm{PTX}=57 \% \pm 4.4 \% \text { (6 mice }) \\
\text { ON: } 100 \mathrm{nl} \mathrm{PTX}=33 \% \pm 2.4 \%(6 \text { mice }) \\
\text { ON: } 200 \mathrm{nl} \mathrm{PTX}=6.4 \% \pm 1 \%(6 \text { mice })\end{array}$ & $\begin{array}{l}\text { Paired Student t-test } \\
\text { Paired Student t-test }\end{array}$ & $\begin{array}{l}\text { Time for attack during ON: PTX } 100 \mathrm{nl} \text { vs. } 0 \mathrm{nl}: \mathrm{P}=7.29528 \mathrm{E}-4 \text { *** } \\
\text { Time for attack during ON: PTX } 200 \mathrm{nl} \text { vs. } 0 \mathrm{nl}: \mathrm{P}=5.43005 \mathrm{E}-7^{\text {***}}\end{array}$ \\
\hline $5 \mathrm{H}$ & 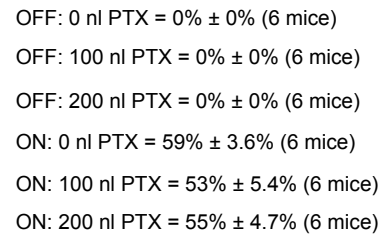 & $\begin{array}{l}\text { Paired Student t-test } \\
\text { Paired Student t-test }\end{array}$ & $\begin{array}{l}\text { Time for attack: PTX } 100 \mathrm{nl} \text { vs. } 0 \mathrm{nl}: \mathrm{P}=0.40445 \mathrm{n} . \mathrm{s} . \\
\text { Time for attack: PTX } 200 \mathrm{nl} \text { vs. } 0 \mathrm{nl}: \mathrm{P}=0.51817 \text { n.s. }\end{array}$ \\
\hline & $\begin{array}{l}\text { GtACR1-OFF }=5.58 \pm 0.69(7 \text { mice }) \\
\text { GtACR1-ON }=4.07 \pm 0.58(7 \text { mice })\end{array}$ & Paired Student t-test & Biting bout number: ON vs. OFF: $\mathrm{P}=8.14002 \mathrm{E}-5^{* \star *}$ \\
\hline & $\begin{array}{l}\text { EGFP-OFF }=4.70 \pm 0.50(7 \text { mice }) \\
\text { EGFP-ON }=4.72 \pm 0.37(7 \text { mice })\end{array}$ & Paired Student t-test & Biting bout number: ON vs. OFF: $P=0.91959$ n.s. \\
\hline & $\begin{array}{l}\text { GtACR1-OFF = } 433 \pm 53 \text { (7 mice }) \\
\text { GtACR1-ON = } 346 \pm 52 \text { (7 mice })\end{array}$ & Paired Student t-test & Total biting time: ON vs. OFF: $P=0.00178^{* *}$ \\
\hline $5 \mathrm{~K}$ & $\begin{array}{l}\text { EGFP-OFF }=417 \pm 49(7 \text { mice }) \\
\text { EGFP-ON }=400 \pm 44(7 \text { mice })\end{array}$ & Paired Student t-test & Total biting time: ON vs. OFF: $P=0.34201$ n.s. \\
\hline
\end{tabular}




\section{Table S4 - 3 Summary of statistical analyses}

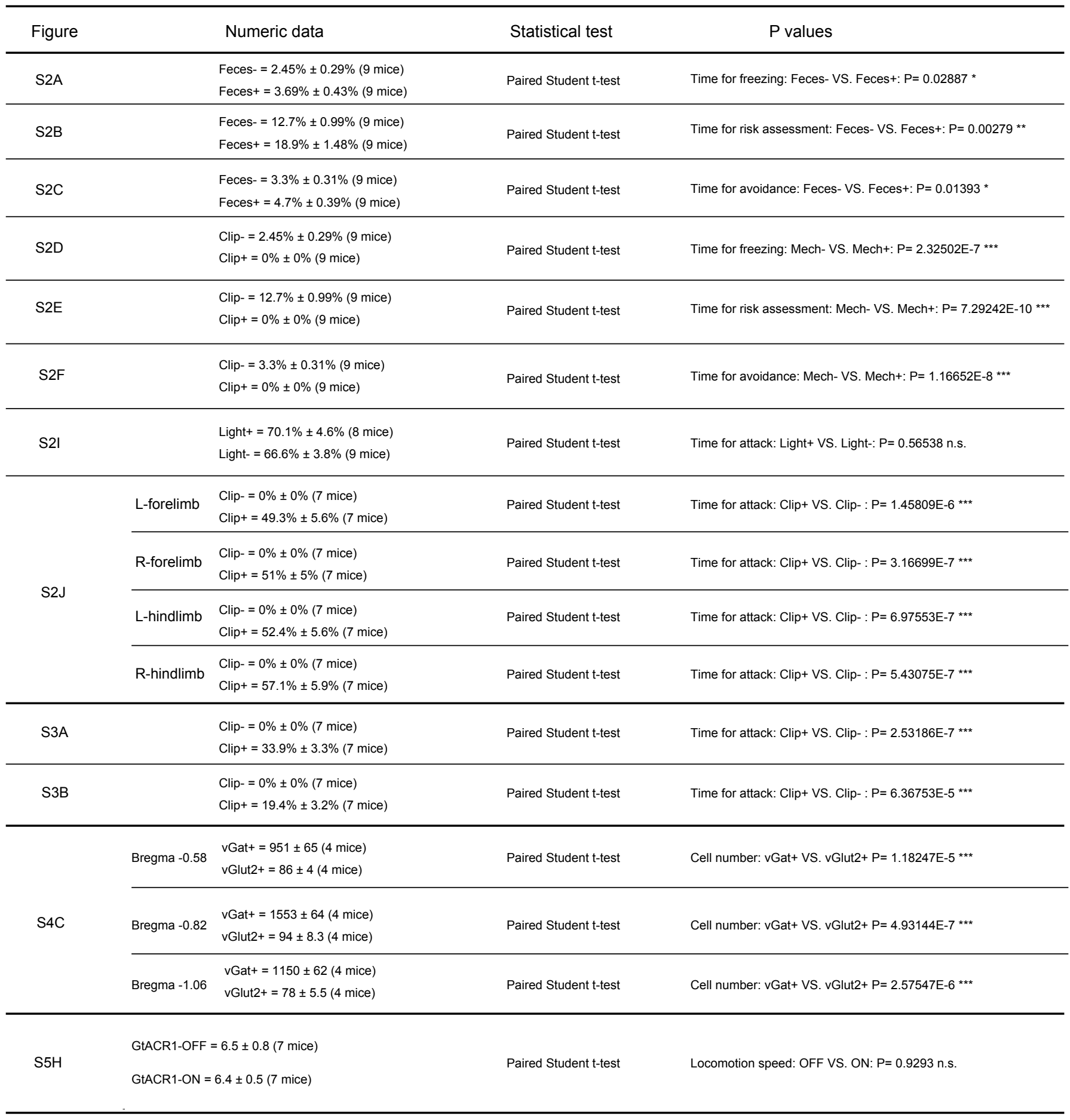




\section{Table S4 - 4 Summary of statistical analyses}

\begin{tabular}{|c|c|c|c|}
\hline Figure & Sample size (n) & Statistical test & $P$ values \\
\hline S9B & $\begin{array}{l}\text { ChR2-OFF }=2.61 \% \pm 0.38 \%(7 \text { mice }) \\
\text { ChR2-ON }=0 \% \pm 0 \%(7 \text { mice })\end{array}$ & Paired Student t-test & Time for avoidance: ON vs. OFF: $\mathrm{P}=4.41441 \mathrm{E}-4$ *** \\
\hline S9C & $\begin{array}{l}\text { ChR2-OFF }=7.1 \% \pm 1.1 \%(7 \text { mice }) \\
\text { ChR2-ON }=0 \% \pm 0 \%(7 \text { mice })\end{array}$ & Paired Student t-test & Time for freezing: ON vs. OFF: $P=6.30155 E-4$ *** \\
\hline S9D & $\begin{array}{l}5 \mathrm{~Hz}=3.6 \% \pm 1.5 \%(7 \text { mice }) \\
10 \mathrm{~Hz}=23 \% \pm 2 \%(7 \text { mice }) \\
20 \mathrm{~Hz}=64.6 \% \pm 3.6 \% \text { (7 mice })\end{array}$ & $\begin{array}{l}\text { Paired Student t-test } \\
\text { Paired Student t-test }\end{array}$ & $\begin{array}{l}\text { Time for anti-predator attack: } 10 \mathrm{~Hz} \text { VS. } 5 \mathrm{~Hz}: \mathrm{P}=3.82493 \mathrm{E}-5^{* * *} \\
\text { Time for anti-predator attack: } 20 \mathrm{~Hz} \text { VS. } 10 \mathrm{~Hz}: \mathrm{P}=7.28439 \mathrm{E}-6^{* * *}\end{array}$ \\
\hline S9E & $\begin{array}{l}2 \mathrm{~mW}=6.2 \% \pm 0.5 \%(7 \text { mice }) \\
5 \mathrm{~mW}=34 \% \pm 2.1 \%(7 \text { mice }) \\
10 \mathrm{~mW}=64.6 \% \pm 3.6 \% \text { ( } 7 \text { mice })\end{array}$ & $\begin{array}{l}\text { Paired Student t-test } \\
\text { Paired Student t-test }\end{array}$ & $\begin{array}{l}\text { Time for anti-predator attack: } 5 \mathrm{~mW} \text { VS. } 2 \mathrm{~mW}: \mathrm{P}=4.32547 \mathrm{E}-5^{* *} \\
\text { Time for anti-predator attack: } 10 \mathrm{~mW} \text { VS. } 5 \mathrm{~mW}: \mathrm{P}=6.218956 \mathrm{E}-6 \text { ** }\end{array}$ \\
\hline \multirow{2}{*}{ S9G } & $\begin{array}{ll}\text { No target } & \text { ChR2-OFF }=0 \pm 0(5 \text { mice }) \\
& \text { ChR2-ON }=0 \pm 0(5 \text { mice })\end{array}$ & Paired Student t-test & Biting bout number: ON vs. OFF: $P$ value can not be calculated \\
\hline & $\begin{array}{l}\text { ChR2-OFF }=0 \pm 0(5 \text { mice }) \\
\text { ChR2-ON }=2.8 \pm 0.37(5 \text { mice })\end{array}$ & Paired Student t-test & Biting bout number: ON vs. OFF: $P=0.00171 * *$ \\
\hline s9J & $\begin{array}{l}\text { ChR2-OFF }=0 \% \pm 0 \%(7 \text { mice }) \\
\text { ChR2-ON }=14 \% \pm 2.9 \% \text { (7 mice })\end{array}$ & Paired Student t-test & Time for attack: ON vs. OFF: $\mathrm{P}=0.00314$ ** \\
\hline S9K & $\begin{array}{l}\text { ChR2-OFF }=13.5 \% \pm 0.94 \%(7 \text { mice }) \\
\text { ChR2-ON }=20.6 \% \pm 1.6 \%(7 \text { mice })\end{array}$ & Paired Student t-test & Time for exploration: ON vs. OFF: $\mathrm{P}=8.52751 \mathrm{E}-4$ *** \\
\hline $\mathrm{S} 10 \mathrm{C}$ & $\begin{array}{l}\text { ChR2-OFF }=0 \% \pm 0 \% \text { (7 mice }) \\
\text { ChR2-ON }=0 \% \pm 0 \% \text { (7 mice })\end{array}$ & Paired Student t-test & Social attack: ON vs. OFF: P value can not be calculated \\
\hline S10D & $\begin{array}{l}\text { ChR2-OFF }=0 \% \pm 0 \% \text { (7 mice }) \\
\text { ChR2-ON }=0 \% \pm 0 \% \text { (7 mice })\end{array}$ & Paired Student t-test & Mounting: ON vs. OFF: P value can not be calculated \\
\hline S10E & $\begin{array}{l}\text { ChR2-OFF }=51 \% \pm 5.1 \%(7 \text { mice }) \\
\text { ChR2-ON }=56 \% \pm 5.6 \%(7 \text { mice })\end{array}$ & Paired Student t-test & Social investigation: ON vs. OFF: $P=0.039$ * \\
\hline $\mathrm{S} 10 \mathrm{H}$ & $\begin{array}{l}\text { ChR2-OFF }=0 \% \pm 0 \% \text { (7 mice }) \\
\text { ChR2-ON }=0 \% \pm 0 \%(7 \text { mice })\end{array}$ & Paired Student t-test & Social attack: ON vs. OFF: P value can not be calculated \\
\hline S10l & $\begin{array}{l}\text { ChR2-OFF }=0 \% \pm 0 \% \text { (7 mice }) \\
\text { ChR2-ON }=0 \% \pm 0 \%(7 \text { mice })\end{array}$ & Paired Student t-test & Mounting: ON vs. OFF: $P$ value can not be calculated \\
\hline S10J & $\begin{array}{l}\text { ChR2-OFF }=63.6 \% \pm 3.7 \% \text { (7 mice }) \\
\text { ChR2-ON }=72.7 \% \pm 4.1 \%(7 \text { mice })\end{array}$ & Paired Student t-test & Social investigation: ON vs. OFF: $P=0.00664$ ** \\
\hline
\end{tabular}




\section{Table S4 - 5. Summary of statistical analyses}

\begin{tabular}{|c|c|c|c|}
\hline Figure & Numeric Data & Statistical test & $P$ values \\
\hline S11B & $\begin{array}{l}\text { ChR2-OFF }=0 \% \pm 0 \%(7 \text { mice }) \\
\text { ChR2-ON }=0 \% \pm 0 \% \text { (7 mice })\end{array}$ & Paired Student t-test & Social attack: ON VS. OFF: P value can not be calculated \\
\hline S11C & $\begin{array}{l}\text { ChR2-OFF }=6.2 \% \pm 0.64 \%(7 \text { mice }) \\
\text { ChR2-ON }=0 \% \pm 0 \%(7 \text { mice })\end{array}$ & Paired Student t-test & Risk assessment: ON VS. OFF: $\mathrm{P}=7.4517 \mathrm{E}-5^{* * *}$ \\
\hline S11D & $\begin{array}{l}\text { ChR2-OFF }=1.3 \% \pm 0.17 \%(7 \text { mice }) \\
\text { ChR2-ON }=0 \% \pm 0 \%(7 \text { mice })\end{array}$ & Paired Student t-test & Avoidance: ON VS. OFF: $\mathrm{P}=2.53096 \mathrm{E}-4$ *** \\
\hline $\mathrm{S} 11 \mathrm{~F}$ & $\begin{array}{l}\text { ChR2-OFF }=0 \% \pm 0 \%(7 \text { mice }) \\
\text { ChR2-ON }=0 \% \pm 0 \%(7 \text { mice })\end{array}$ & Paired Student t-test & Social attack: ON VS. OFF: P value can not be calculated \\
\hline S11G & $\begin{array}{l}\text { ChR2-OFF }=3.8 \% \pm 0.5 \% \text { ( } 7 \text { mice }) \\
\text { ChR2-ON }=0 \% \pm 0 \%(7 \text { mice })\end{array}$ & Paired Student t-test & Risk assessment: ON VS. OFF: $\mathrm{P}=3.75143 \mathrm{E}-4$ *** \\
\hline $\mathrm{S} 11 \mathrm{H}$ & $\begin{array}{l}\text { ChR2-OFF }=0.6 \% \pm 0.07 \%(7 \text { mice }) \\
\text { ChR2-ON }=0 \% \pm 0 \%(7 \text { mice })\end{array}$ & Paired Student t-test & Avoidance: ON VS. OFF: $\mathrm{P}=1.47974 \mathrm{E}-4$ *** \\
\hline $\mathrm{S} 13 \mathrm{C}$ & $\begin{array}{l}\text { ChR2-OFF }=9.8 \% \pm 1.3 \%(7 \text { mice }) \\
\text { ChR2-ON }=0 \% \pm 0 \%(7 \text { mice })\end{array}$ & Paired Student t-test & Freezing: ON VS. OFF: $\mathrm{P}=2.4948 \mathrm{E}-4$ *** \\
\hline S13D & $\begin{array}{l}\text { ChR2-OFF }=11.9 \% \pm 1.01 \%(7 \text { mice }) \\
\text { ChR2-ON }=0 \% \pm 0 \% \text { (7 mice })\end{array}$ & Paired Student t-test & Risk assessment: ON VS. OFF: $P=2.28889 \mathrm{E}-5$ *** \\
\hline S13E & $\begin{array}{l}\text { ChR2-OFF }=3.04 \% \pm 0.22 \%(7 \text { mice }) \\
\text { ChR2-ON }=0 \% \pm 0 \%(7 \text { mice })\end{array}$ & Paired Student t-test & Avoidance: ON VS. OFF: $\mathrm{P}=1.02889 \mathrm{E}-5$ *** \\
\hline S14E & $\begin{array}{l}\text { ChR2-OFF }=0 \% \pm 0 \% \text { (7 mice }) \\
\text { ChR2-ON }=0 \% \pm 0 \%(7 \text { mice })\end{array}$ & Paired Student t-test & Anti-predator attack: ON VS. OFF: P value can not be calculated \\
\hline S14F & $\begin{array}{l}\text { ChR2-OFF }=8.5 \% \pm 1.16 \%(7 \text { mice }) \\
\text { ChR2-ON }=0 \% \pm 0 \%(7 \text { mice })\end{array}$ & Paired Student t-test & Freezing: ON VS. OFF: $\mathrm{P}=3.23427 \mathrm{E}-4$ *** \\
\hline S14G & $\begin{array}{l}\text { ChR2-OFF }=10.6 \% \pm 0.8 \% \text { (7 mice }) \\
\text { ChR2-ON }=16.5 \% \pm 1.1 \%(7 \text { mice })\end{array}$ & Paired Student t-test & Risk assessment: ON VS. OFF: $\mathrm{P}=0.00235^{\star *}$ \\
\hline $\mathrm{S} 14 \mathrm{H}$ & $\begin{array}{l}\text { ChR2-OFF }=2.45 \% \pm 0.28 \% \text { (7 mice }) \\
\text { ChR2-ON }=4.08 \% \pm 0.26 \% \text { (7 mice })\end{array}$ & Paired Student t-test & Avoidance: ON VS. OFF: $\mathrm{P}=5.35646 \mathrm{E}-4$ *** \\
\hline
\end{tabular}


A

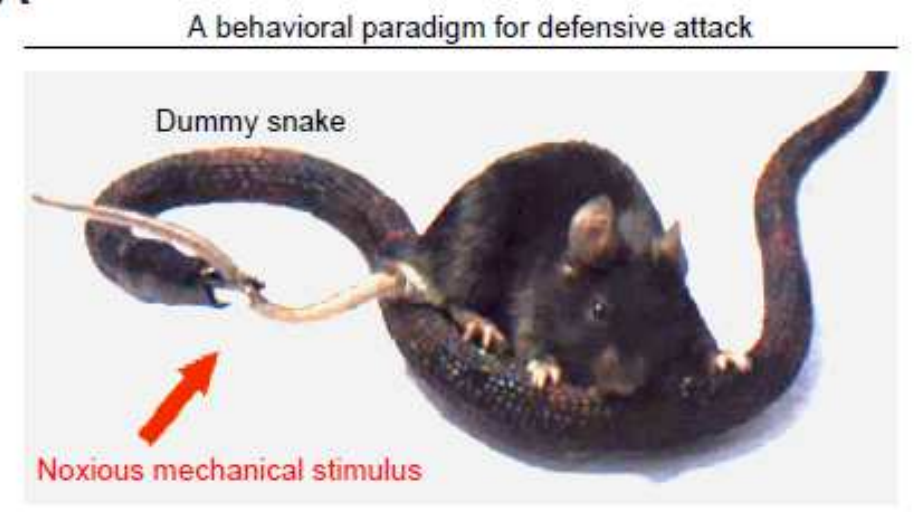

B

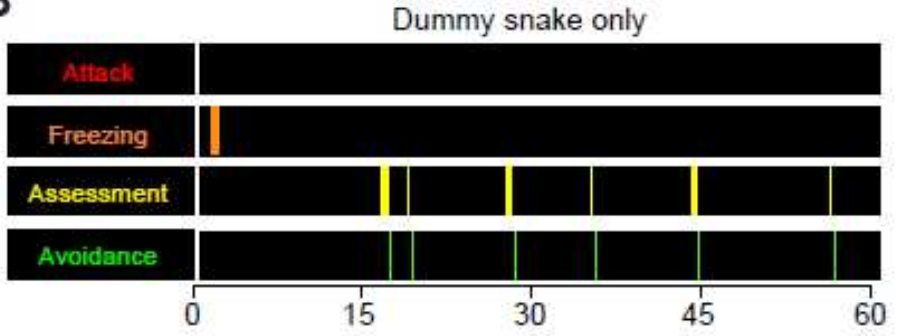

C

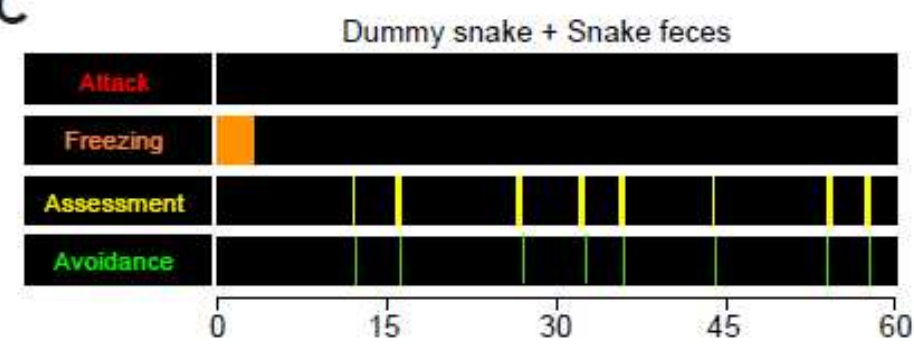

D

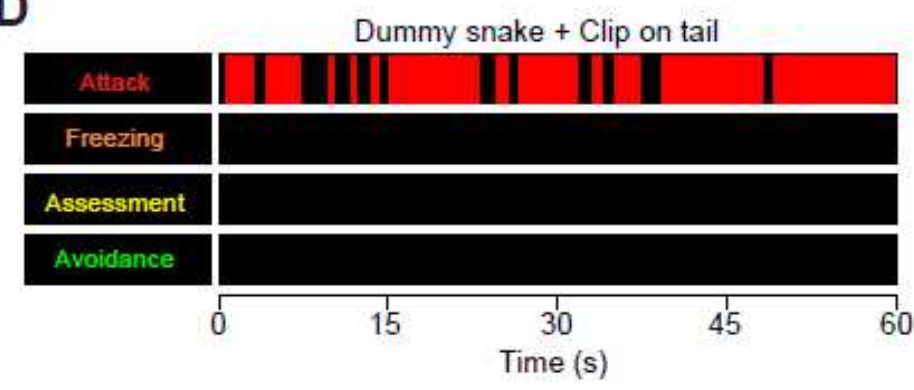

E

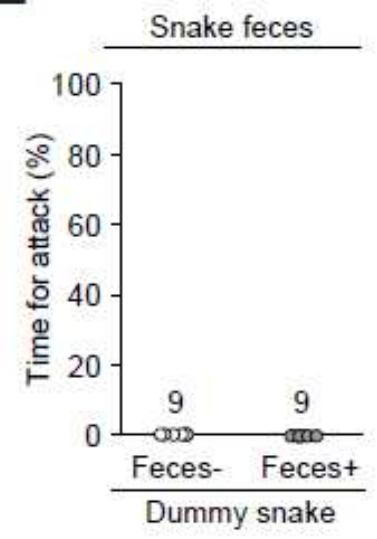

F

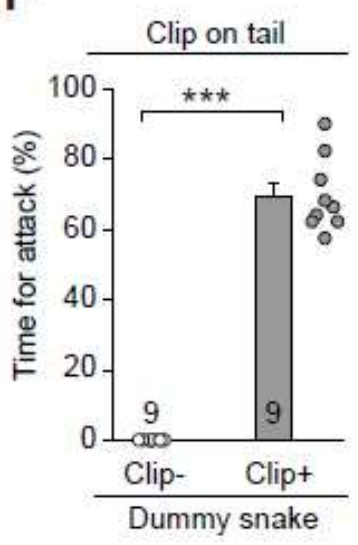

G

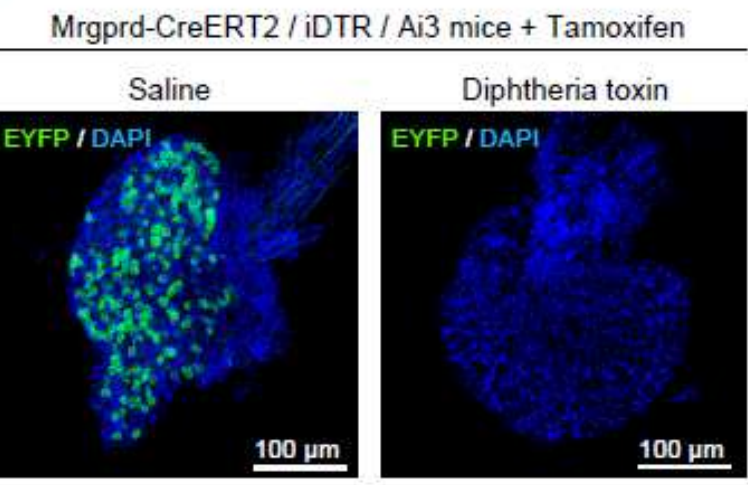

$\mathrm{H}$

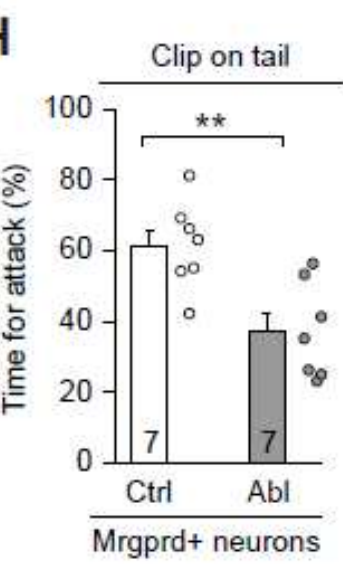

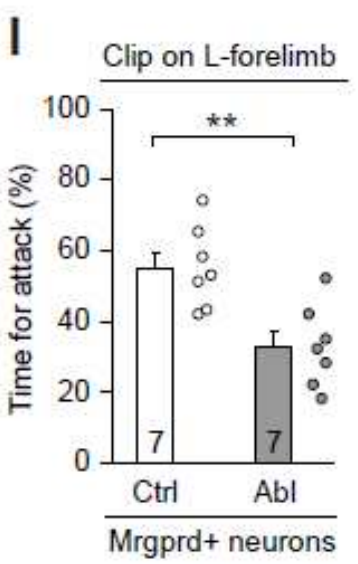

\section{Figure 1}

Noxious mechanical stimulus to evoke defensive attack in mice. (A) An example picture showing a plastic dummy snake equipped with an alligator-clip to apply noxious mechanical stimulus (arrow) to mouse tail. (B-D) Behavioral ethograms of WT mice exposed to a dummy snake only (B), a dummy snake coated with snake feces $(C)$, and a dummy snake equipped with an alligator-clip to apply noxious 
mechanical stimulus to mouse tail (D). The colored bars in the ethograms indicated the onset and offset of specific behaviors. (E) Time spent for attack by biting the dummy snake with and without snake feces. (F) Time spent for attack by biting the dummy snake with and without the alligator-clip to apply noxious mechanical stimulus. (G) Example micrographs of DRG of Mrgprd-CreERT2/iDTR/Ai3 mice treated with saline or Diphtheria toxin. $(\mathrm{H}, \mathrm{l})$ Time spent for attack in mice with and without ablation of putative Mrgprd+ DRG neurons evoked by noxious mechanical stimulus on tail $(H)$ or on left forelimb (I). Number of mice was indicated in the graphs $(E, F, H, I)$. Data in $(E, F, H, I)$ are means $\pm S E M$ (error bars). Statistical analyses in $(E, F, H, I)$ were performed by Student t-tests $(* \star P<0.01 ; * \star \star P<0.001)$. For the $P$ values, see Table S4.

A

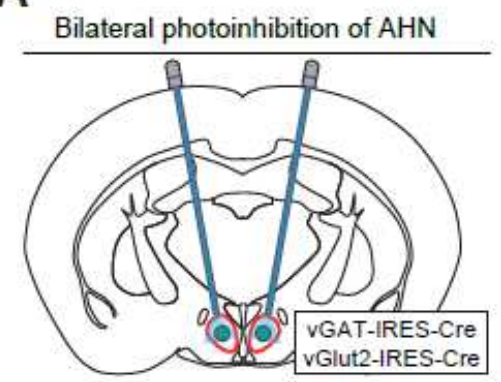

B

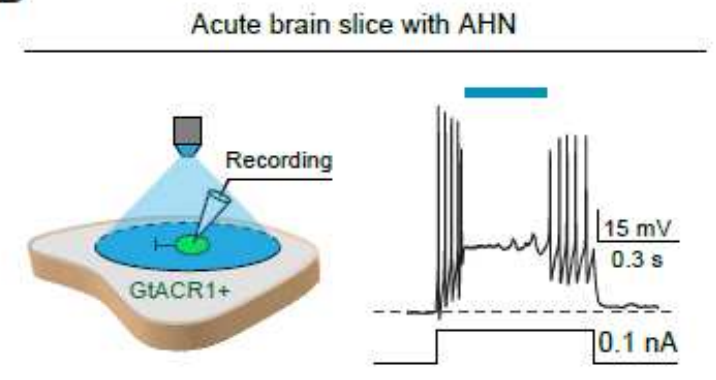

C

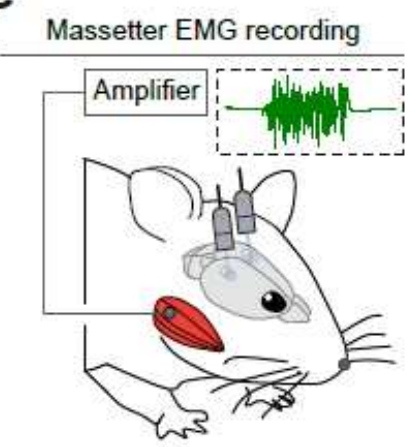

F

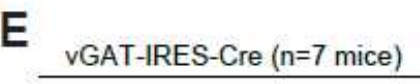

D

vGAT-IRES-Cre

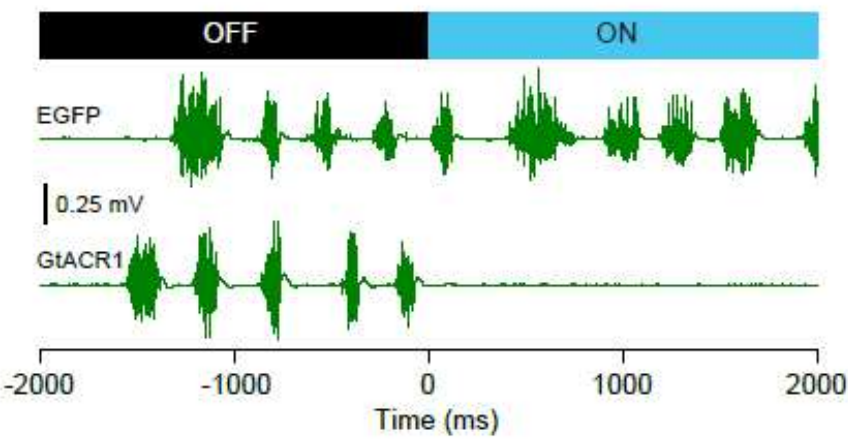

G
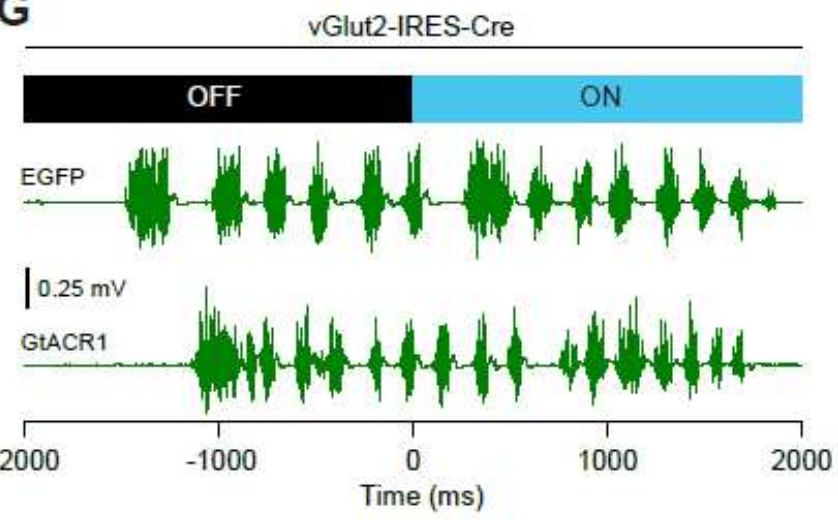

E

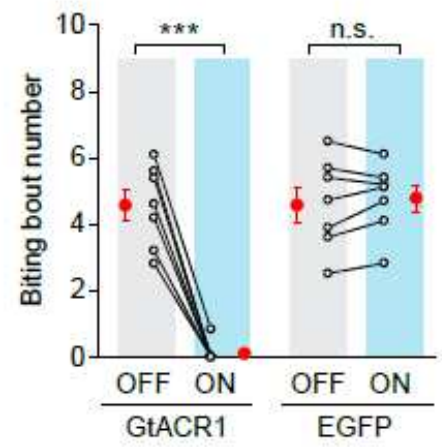

H

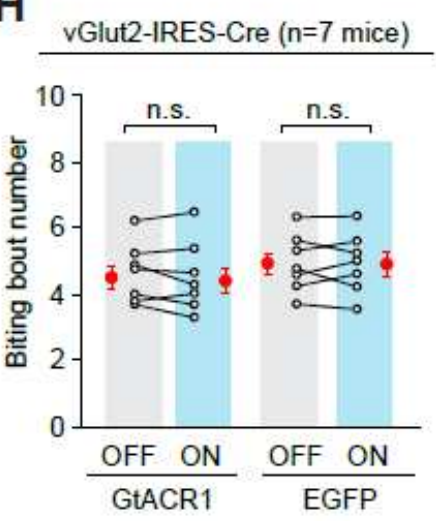

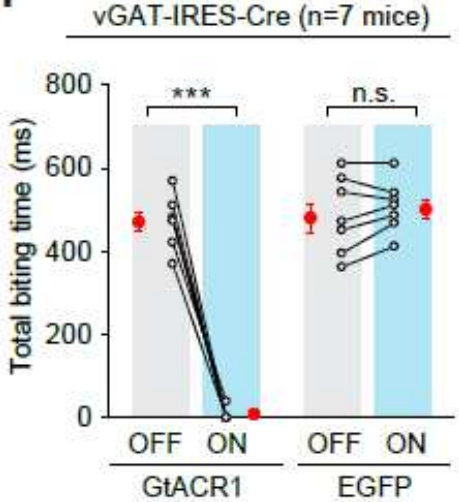

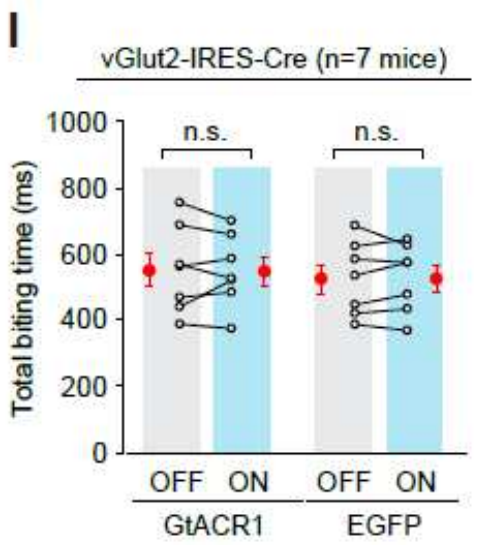


AHN vGAT+ neurons are required for mechanically-evoked defensive attack. (A) Schematic diagram of bilateral photoinhibition of AHN vGAT+ or vGlut2+ neurons. For the micrographs with the optic fiber tracks, see Figure S5A and S5B. For the analyses of cell-type specificity, see Figure S5C-S5F. (B) Schematic diagram (left) and example trace (right) showing photoinhibition of AHN neurons expressing GtACR1. (C) Schematic diagram showing EMG recording from masseter muscles of mice. (D-F) Example traces $(D)$ and quantitative analyses of biting bout number $(E)$ and total biting time $(F)$ within laser OFF phase and ON phase, showing the effect of photoinhibition of VGAT+ AHN neurons on mechanicallyevoked biting attack. Mice with EGFP expressed in AHN vGAT+ neurons were used as a control for GtACR1. (G-I) Example traces $(\mathrm{G})$ and quantitative analyses of biting bout number $(\mathrm{H})$ and total biting time (I) within phases of laser OFF and ON, showing the effect of photoinhibition of AHN vGlut2+ neurons on mechanically-evoked biting attack. Mice with EGFP expressed in AHN vGlut2+ neurons were used as a control for GtACR1. For the effect of photoinhibition of AHN vGAT+ neurons on locomotion speed of freely-moving mice, see Figure $S 5 H$. Number of mice was indicated in the graphs $(E, F, H, I)$. Data in $(E, F$, $H, I)$ are means \pm SEM (error bars). Statistical analyses in $(E, F, H, I)$ were performed by Student t-tests (n.s. $P>0.1, * \star * P<0.001)$. For the $P$ values, see Table $S 4$. 


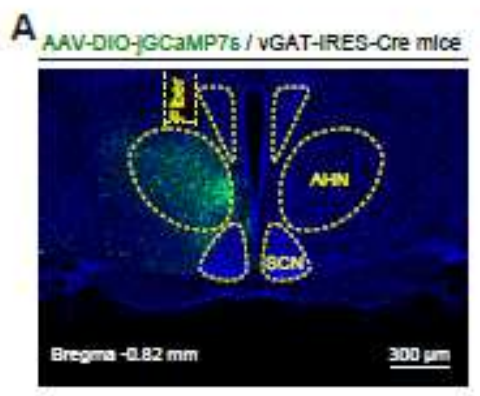

B

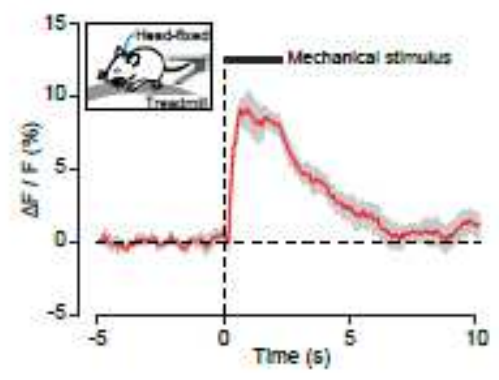

C

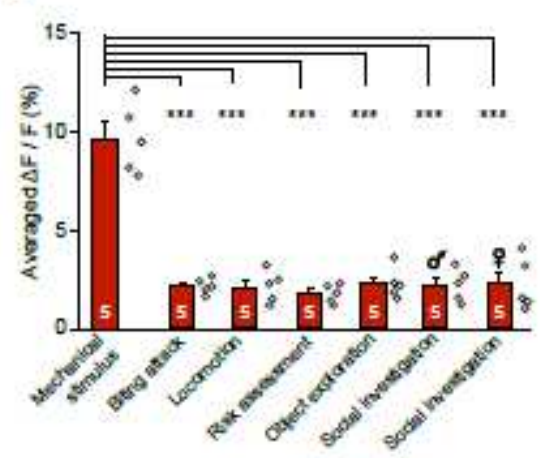

D

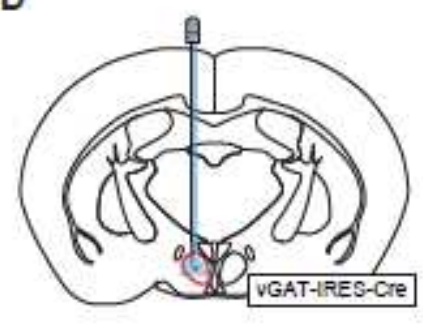

E

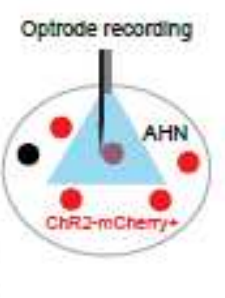

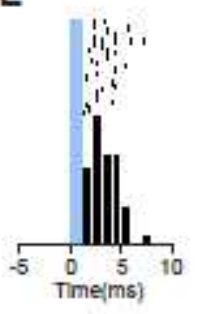

F

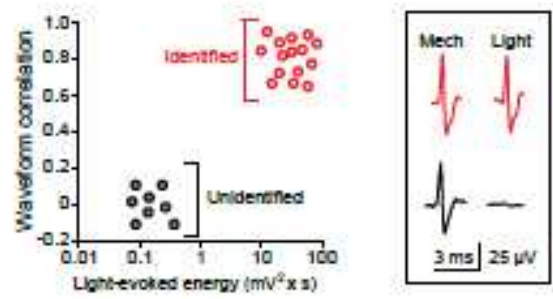

G

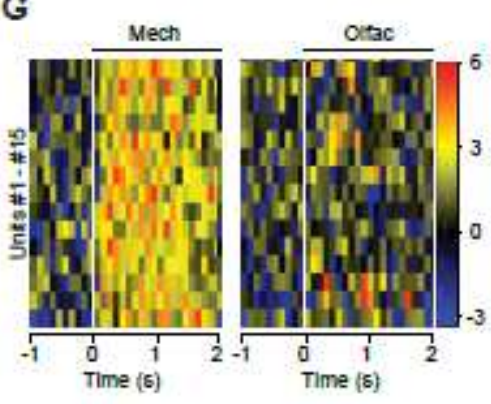

I

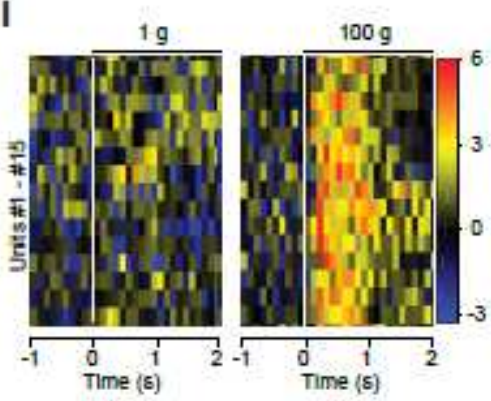

H
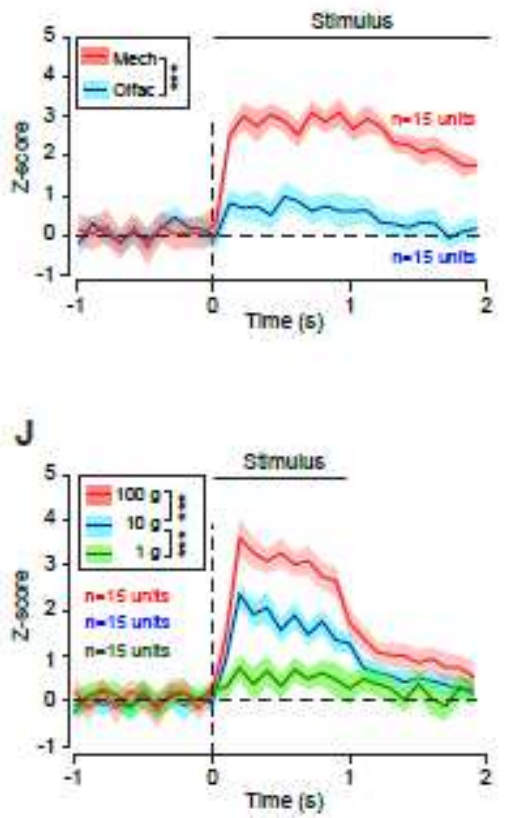

K
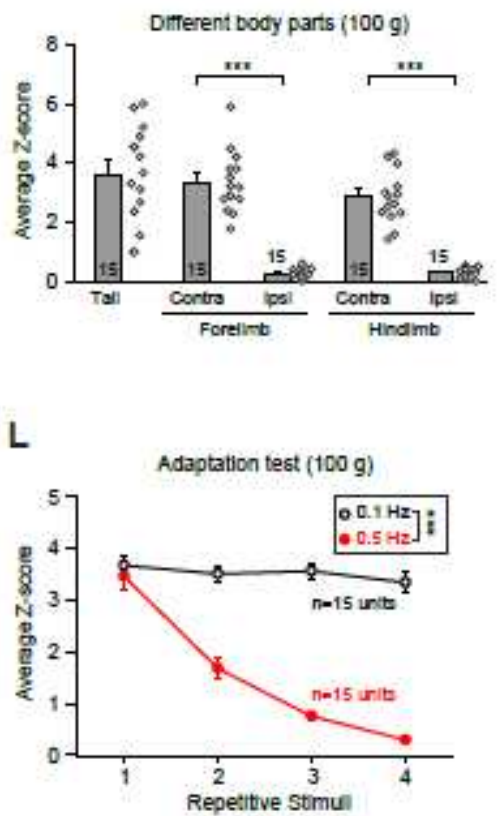

\section{Figure 3}

AHN vGAT+ neurons encode mechanical stimuli. (A) An example micrograph showing jGCaMP7s expressed in the AHN of vGAT-IRES-Cre mice and the optical fiber track above the AHN. For the analyses of cell-type specificity of jGCaMP7s expression, see Figure S6A and S6B. (B) Normalized GCaMP fluorescence changes $(\Delta F / F)$ in response to noxious mechanical stimulus on the tail. Inset, the head-fixed awake mouse standing on a treadmill was applied with noxious mechanical stimulus by tail clamping with an alligator-clip. (C) Quantitative analyses of peak GCaMP responses of AHN vGAT+ neurons in 
seven behavioral tests. The example trace for the test of "Mechanical stimulus" was in Figure 3B, while the other six example traces were in Figure S6C-S6H. (D) Schematic diagram of optrode recording from AHN vGAT+ neurons expressing ChR2-mCherry. For an example micrograph showing the optical fiber track and electrolytic lesion of recording site in the AHN, see Figure S7A. The principal component analysis for spike sorting of an example unit was in Figure S7B. (E) Raster and peri-stimulus time histogram (PSTH) of an example putative AHN vGAT+ neuron with spiking latency less than 5 ms relative to the onset of light pulses. (F) Correlation analysis of action potentials of individual units evoked either by light pulses (Light) or by mechanical stimuli (Mech), confirming a segregation between optogenetically identified units (Identified, red) and unidentified units (Unidentified, grey). (G) Heat-map PSTH of Z-scored firing rates of individual AHN vGAT+ neurons to mechanical stimuli (Mech) or olfactory stimuli (Olfac). For the schematic diagrams showing the application of mechanical stimuli and olfactory stimuli to the test mice, see Figure S7C and S7E. For the example units of putative AHN vGAT+ neurons in response to mechanical stimuli and olfactory stimuli, see Figure S7D and S7F. (H) Average PSTH of Z-scored firing rates of all identified AHN vGAT+ neurons to mechanical stimuli (Mech, red) and olfactory stimuli (Olfac, blue). (I) Heat-map PSTH of Z-scored firing rates of individual AHN vGAT+ neurons to mechanical stimuli applied with von Frey filaments $(1 \mathrm{~g}, 100 \mathrm{~g})$. For the schematic diagram showing the application of von Frey filaments to the test mice, see Figure S7G. For the heat-map PSTH of Z-scored firing rates of individual AHN vGAT+ neurons to $10 \mathrm{~g}$ mechanical stimuli, see Figure S7H. (J) Average PSTH of Z-scored firing rates of all identified AHN vGAT+ neurons to mechanical force with different intensities. (K) Averaged peak Z-scored firing rates of all identified AHN vGAT+ neurons to mechanical stimuli $(100 \mathrm{~g})$ on different body parts. ( $L$ ) Averaged peak Z-scored firing rates of all identified AHN vGAT+ neurons to four repetitive mechanical stimuli $(100 \mathrm{~g})$ applied at $0.1 \mathrm{~Hz}$ and $0.5 \mathrm{~Hz}$ on the tail. Number of mice (C) and number of units $(H, J, K, L)$ were indicated in the graphs. Data in $(B, C, H, J, K, L)$ are means $\pm S E M$ (error bars). Statistical analyses were performed by Student t-tests $(C, K)$ and One-Way ANOVA $(H, J, L)(* \star *)<$ 0.001). For the $P$ values, see Table $S 4$. 

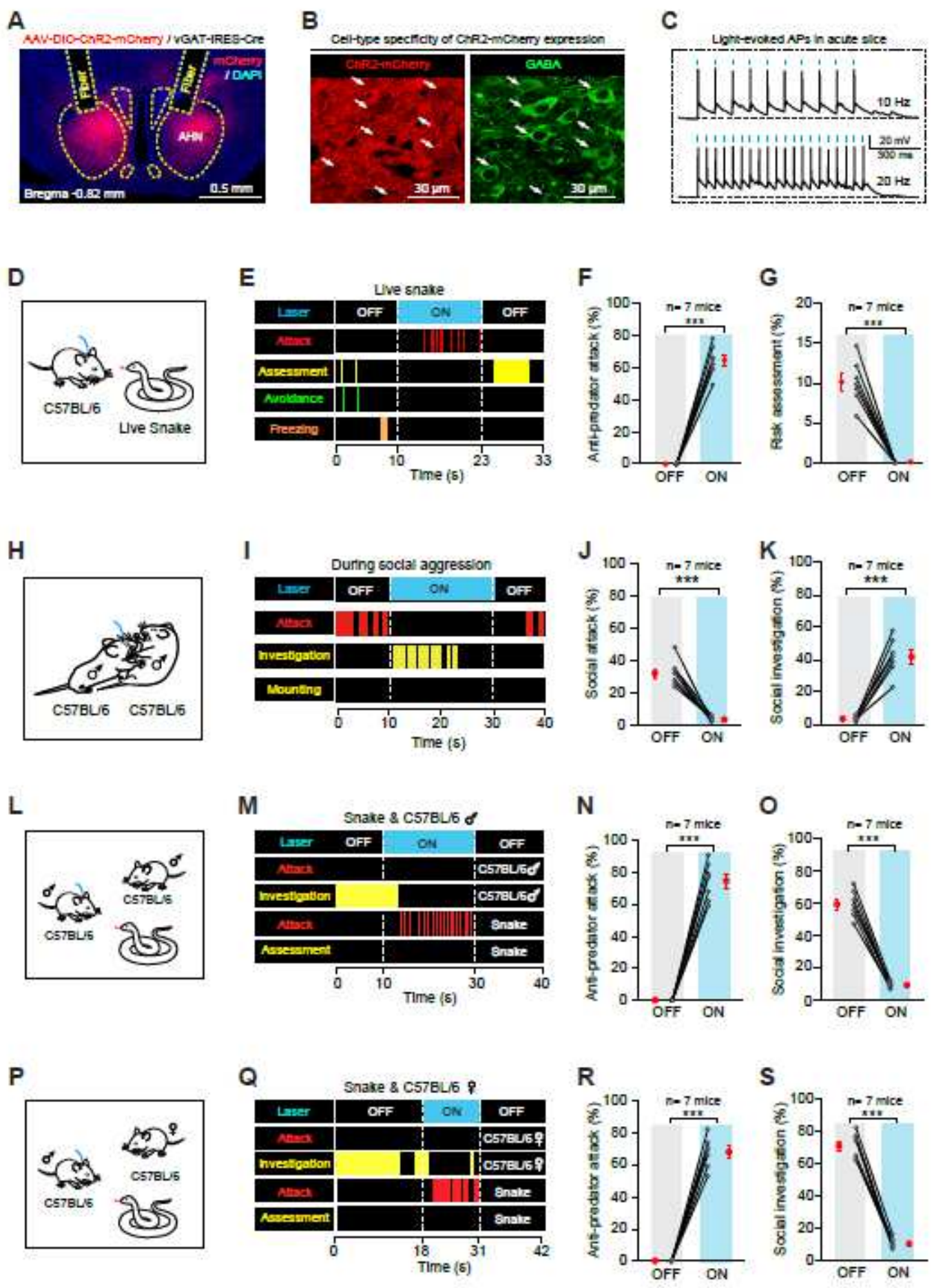

\section{Figure 4}

Activation of AHN vGAT+ neurons trigger biting attack to non-social targets. (A) An example coronal section showing ChR2-mCherry expression in the AHN of vGAT-IRES-Cre mice and bilateral optical fiber tracks above the AHN. (B) Example micrographs showing the cell-type specificity of ChR2-mCherry expression in GABA+ AHN neurons. Arrows indicated soma of GABA+ cells expressing ChR2-mCherry. For the quantitative analysis, see Figure S9A. (C) Light-pulse trains $(473 \mathrm{~nm}, 2 \mathrm{~ms}, 10 \mathrm{~mW}, 10 \mathrm{~Hz}$ or $20 \mathrm{~Hz})$ reliably evoked phase-locked spiking activity in ChR2-mCherry+ AHN cells. (D) Schematic diagram 
showing a test mouse confronted with a live snake in the arena. (E-G) Example behavioral ethogram (E), quantitative analyses of time for anti-predator attack $(F)$ and time for risk assessment $(G)$ of mice before (OFF) and during (ON) photostimulation of AHN vGAT+ neurons. For quantitative analyses of time for freezing and avoidance, see Figure S9B and S9C. The colored bars in the ethograms indicated the onset and offset of specific behaviors. $(\mathrm{H})$ Schematic diagram showing a test mouse exhibiting social aggression toward a male intruder. (I-K) Example behavioral ethogram (I), quantitative analyses of time for social attack $(\mathrm{J})$ and time for social investigation (K) of mice before (OFF) and during (ON) photostimulation of AHN vGAT+ neurons. For more behavioral analyses, see Figure S10. (L) Schematic diagram showing a test mouse confronted with a live snake and a male C57BL/6 mouse in the arena. (MO) Example behavioral ethogram (M), quantitative analyses of time for anti-predator attack $(\mathrm{N})$ and time for social investigation (O) of test mice before (OFF) and during (ON) photostimulation of AHN vGAT+ neurons. For more behavioral analyses, see Figure S11 A-S11D. (P) Schematic diagram showing a test mouse confronted with a live snake and a female C57BL/6 mouse in the arena. (Q-S) Example behavioral ethogram $(Q)$, quantitative analyses of time for anti-predator attack $(R)$ and time for social investigation (S) of test mice before (OFF) and during (ON) photostimulation of AHN vGAT+ neurons. For more behavioral analyses, see Figure S11E-S11H. Number of mice was indicated in the graphs (F, G, J, K, N, O, $R, S)$. Data in ( $F, G, J, K, N, O, R, S)$ are means $\pm S E M$ (error bars). Statistical analyses in $(F, G, J, K, N, O, R$, $S)$ were performed by Student t-tests ( $\left.{ }^{\star \star \star} P<0.001\right)$. For the $P$ values, see Table S4. 

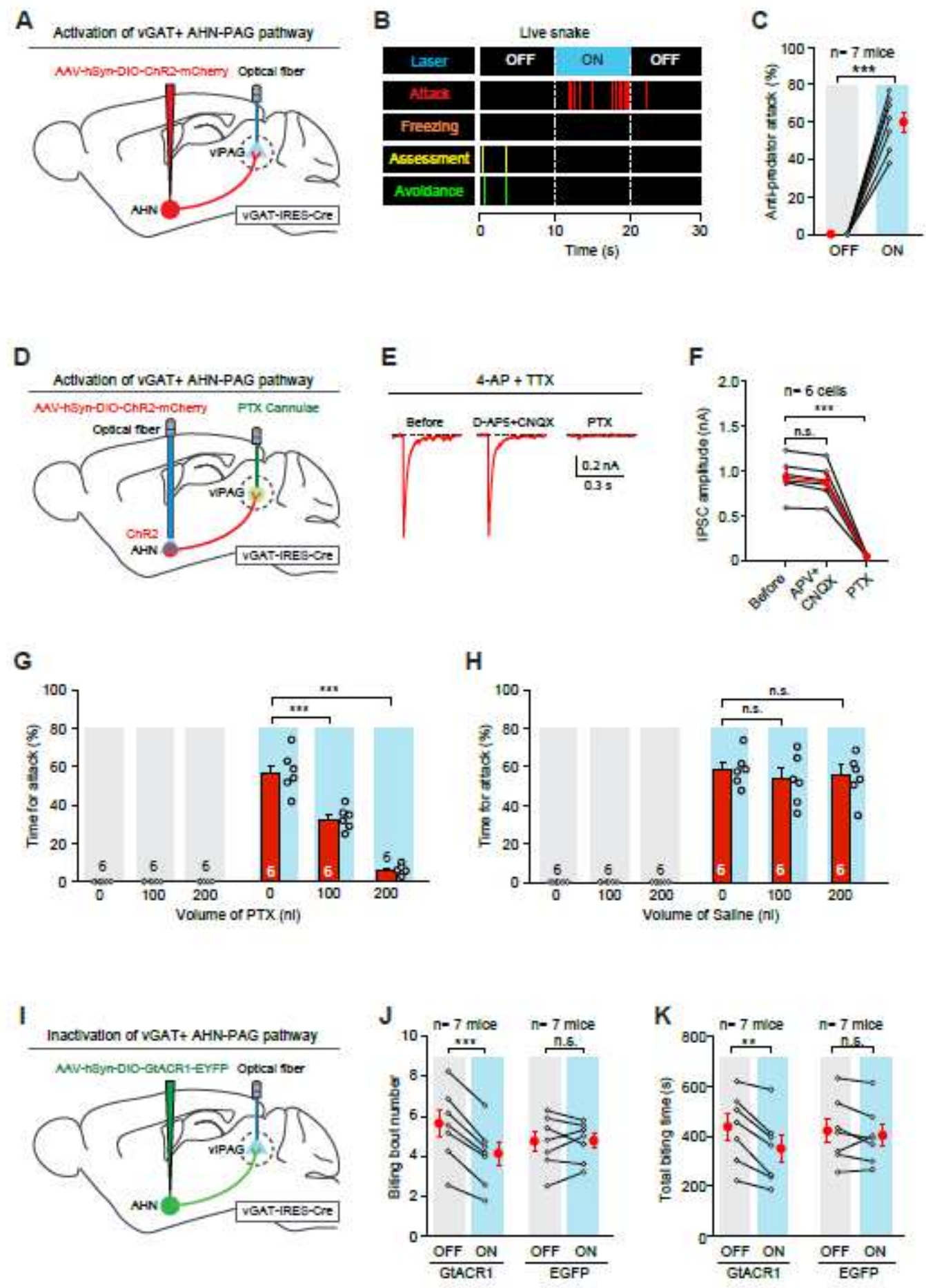

\section{Figure 5}

Role of vGAT+ AHN-PAG pathway 595 for mechanically-evoked defensive attack. (A) Schematic diagram showing AAV injection and optic fiber implantation for light stimulation of vGAT+ AHN-vIPAG pathway. For the example micrographs of the AHN and vIPAG, see Figure S13, A and B. (B, C) Example behavioral ethogram (B) and quantitative analysis of time for anti-predator attack (C) of mice before (OFF) and during (ON) activation of VGAT+ AHN-PAG pathway. For more behavioral analyses, see Figure S13C-S13E. For the analyses of vGAT+ AHN-LS pathway, see Figure S14. The colored bars in the ethograms indicated 
the onset and offset of specific behaviors. (D) Schematic diagram showing AAV injection into the AHN, optical fiber implantation above the AHN, and cannulae implantation above the vIPAG in vGAT-IRES-Cre mice. For the example micrographs with the optic fiber track and cannulae track above the AHN and vIPAG, see Figure S15A and S15B. (E, F) Example traces of postsynaptic currents (PSCs) recorded from vIPAG neurons (E) and quantitative analyses of their amplitude $(F)$ showing the GABAergic action of vGAT+ AHN-PAG pathway is mediated by GABAa receptor. For the schematic diagram for slice physiology, see Figure S15C. $(G, H)$ Quantitative analyses of light-evoked biting attack to live snake in mice with vIPAG treated with different doses of PTX $(G)$ or saline $(H)$. For the example behavioral ethograms, see Figure S15E and S15F. (I) Schematic diagram showing AAV injection and optic fiber implantation for photoinhibition of vGAT+ AHN-vIPAG pathway. For example micrographs of the AHN and vIPAG, see Figure S16A and S16B. (J, K) Quantitative analyses of biting bout number $(\mathrm{J})$ and total biting time $(K)$ toward dummy snake within phases of laser OFF and ON, showing the effect of photoinhibition of VGAT+ AHN-vIPAG pathway on mechanically-evoked biting attack. Mice with EGFP expressed in AHN vGAT+ neurons were used as a control for GtACR1. For example traces, see Figure S16C. Number of mice $(C, G, H, J, K)$ and cells $(F)$ were indicated in the graphs. Data in $(C, F, G, H, J, K)$ are means \pm SEM (error

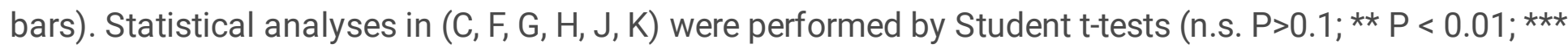
$P<0.001)$. For the $P$ values, see Table $S 4$.

\section{Supplementary Files}

This is a list of supplementary files associated with this preprint. Click to download.

- MovieS1.mp4

- Movies2.mp4

- Movies3.mp4

- Movies4.mp4

- Movies5.mp4

- MovieS6.mp4

- Movies7.mp4

- Movies8.mp4

- Movies9.mp4

- MovieS10.mp4

- MovieS11.mp4

- MovieS12.mp4

- MovieS13.mp4

- MovieS14.mp4

- MovieS15.mp4 